MÉMOIRES DE LA SMF 94

ON MAPPING PROPERTIES OF THE GENERAL RELATIVISTIC CONSTRAINTS OPERATOR IN WEIGHTED FUNCTION SPACES, WITH APPLICATIONS

\author{
Piotr T. Chruściel
}

Erwann Delay

Société Mathématique de France 2003

Publié avec le concours du Centre National de la Recherche Scientifique 
P.T. Chruściel

Département de Mathématiques, Faculté des Sciences, Parc de Grandmont, F-37200 Tours, France.

Current address: Albert Einstein Institute, Golm, Germany.

E-mail : piotr@gargan.math.univ-tours.fr

Url : www.phys.univ-tours.fr/ piotr

E. Delay

Département de Mathématiques, Faculté des Sciences, Parc de Grandmont, F-37200 Tours, France.

E-mail : delay@gargan.math.univ-tours.fr

Url : www.phys.univ-tours.fr $/ \sim$ delay

2000 Mathematics Subject Classification. - 83C05.

Key words and phrases. - General relativistic initial data, non-connected black holes, asymptotically simple space-times, initial data gluing.

P.C.: Partially supported by a Polish Research Committee grant 2 P03B 07324.

E.D.: Partially supported by the ACI program of the French Ministry of Research. 


\title{
ON MAPPING PROPERTIES OF THE GENERAL RELATIVISTIC CONSTRAINTS OPERATOR IN WEIGHTED FUNCTION SPACES, WITH APPLICATIONS
}

\author{
Piotr T. Chruściel, Erwann Delay
}

\begin{abstract}
Generalizing an analysis of Corvino and Schoen, we study surjectivity properties of the constraint map in general relativity in a large class of weighted function spaces. As a corollary we prove several perturbation, gluing, and extension results: we show existence of non-trivial, singularity-free, vacuum space-times which are stationary in a neighborhood of $i^{0}$; for small perturbations of parity-covariant initial data sufficiently close to those for Minkowski space-time this leads to space-times with a smooth global $\mathscr{I}$; we prove existence of initial data for many black holes which are exactly Kerr — or exactly Schwarzschild — both near infinity and near each of the connected components of the apparent horizon; under appropriate conditions we obtain existence of vacuum extensions of vacuum initial data across compact boundaries; we show that for generic metrics the deformations in the Isenberg-Mazzeo-Pollack gluings can be localized, so that the initial data on the connected sum manifold coincide with the original ones except for a small neighborhood of the gluing region; we prove existence of asymptotically flat solutions which are static or stationary up to $r^{-m}$ terms, for any fixed $m$, and with multipole moments freely prescribable within certain ranges.
\end{abstract}

(C) Mémoires de la Société Mathématique de France 94, SMF 2003 
Résumé (Sur les propriétés de l'opérateur de contraintes relativistes dans des espaces à poids, et applications)

Nous étudions les propriétés de surjectivité de l'application de contraintes en relativité générale dans une large classe d'espaces fonctionnels à poids, généralisant ainsi une analyse de Corvino et Schoen. Comme corollaire on obtient plusieurs résultats de perturbation, de recollement, ou d'extension. Ainsi, nous démontrons l'existence d'espaces-temps non triviaux, sans singularités, solutions d'équations d'Einstein du vide, qui sont stationnaires dans un voisinage de $i^{0}$. Pour des données initiales proches de celles de Minkowski ceci conduit, sous une condition de parité approximative, à des espaces-temps avec un infini isotrope $\mathscr{I}$ global et lisse. Nous prouvons l'existence de données initiales pour des trous noirs multiples qui sont exactement kerriennes, ou exactement schwarzschildiennes, dans une région asymptotique, mais aussi près de chaque composante connexe de l'horizon apparent. Nous montrons que pour des métriques génériques les perturbations des données initiales introduites par les recollements du type Isenberg-Mazzeo-Pollack peuvent être localisées, de sorte que les données initiales sur la variété obtenue en prenant la somme connexe coincident avec les données initiales originelles, sauf dans un petit voisinage de la zone de recollement. Nous prouvons l'existence de solutions asymptotiquement plates qui sont statiques ou stationnaires modulo des termes en $r^{-m}$, avec $m$ arbitrairement prescrit, et avec des moments multipolaires qu'on peut spécifier librement dans certains ouverts. 


\section{CONTENTS}

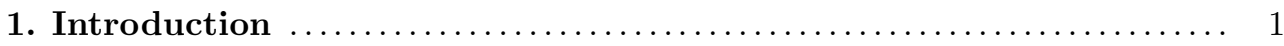

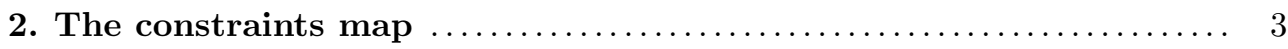

3. Isomorphism theorems $\ldots \ldots \ldots \ldots \ldots \ldots \ldots \ldots \ldots \ldots \ldots \ldots \ldots \ldots \ldots$

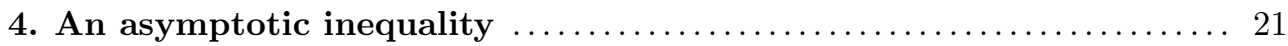

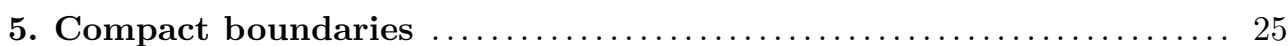

6. Conformally compactifiable initial data $\ldots \ldots \ldots \ldots \ldots \ldots \ldots \ldots \ldots$

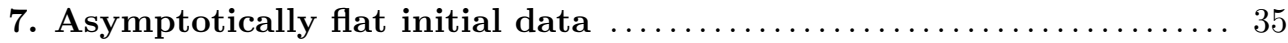

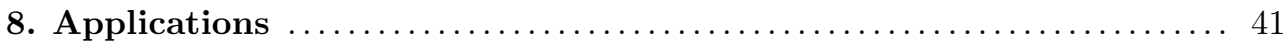

8.1. Space-times that are Kerrian near $i^{0} \ldots \ldots \ldots \ldots \ldots \ldots \ldots \ldots \ldots \ldots \ldots 4$

8.2. Gluing asymptotically flat initial data sets $\ldots \ldots \ldots \ldots \ldots \ldots \ldots \ldots \ldots 4$

8.3. Initial data which are stationary to high asymptotic order $\ldots \ldots \ldots \ldots 4$

8.4. Space-times that are Kerrian near $\mathscr{I}^{+} \ldots \ldots \ldots \ldots \ldots \ldots \ldots \ldots \ldots \ldots$

8.5. Bondi-type asymptotic expansions at $\mathscr{I}^{+} \ldots \ldots \ldots \ldots \ldots \ldots \ldots \ldots \ldots \ldots$

8.6. Local and global extensions of initial data sets $\ldots \ldots \ldots \ldots \ldots \ldots \ldots \ldots . \ldots 1$

8.7. Localized Isenberg-Mazzeo-Pollack gluings $\ldots \ldots \ldots \ldots \ldots \ldots \ldots \ldots \ldots \ldots$

8.8. Vacuum space-times with a smooth global $\mathscr{I} \ldots \ldots \ldots \ldots \ldots \ldots \ldots$

8.9. "Many Kerr" initial data ........................... 59

A. Weighted Sobolev and weighted Hölder spaces $\ldots \ldots \ldots \ldots \ldots \ldots \ldots$

B. Sufficient conditions for the scaling property $\ldots \ldots \ldots \ldots \ldots \ldots \ldots$

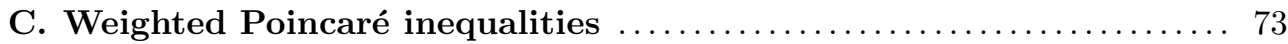

C.1. Application: compact boundaries $\ldots \ldots \ldots \ldots \ldots \ldots \ldots \ldots \ldots \ldots \ldots \ldots . \ldots \ldots$

C.2. Application: asymptotically flat metrics $\ldots \ldots \ldots \ldots \ldots \ldots \ldots \ldots \ldots \ldots 75$

C.3. Application: conformally compact manifolds $\ldots \ldots \ldots \ldots \ldots \ldots \ldots \ldots \ldots .76$ 


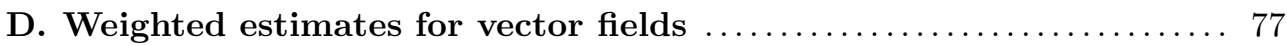

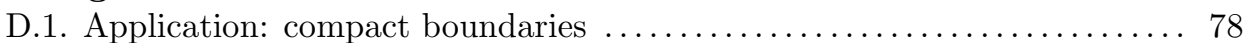

D.2. Application: asymptotically flat metrics $\ldots \ldots \ldots \ldots \ldots \ldots \ldots \ldots \ldots \ldots \ldots$

D.3. Application: conformally compact manifolds $\ldots \ldots \ldots \ldots \ldots \ldots \ldots \ldots$

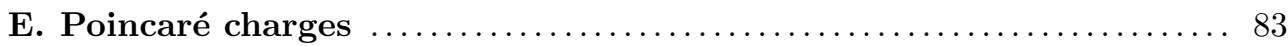

E.1. Initial data asymptotically flat in spacelike directions $\ldots \ldots \ldots \ldots \ldots . . .64$

F. The reference family of Kerr metrics $\ldots \ldots \ldots \ldots \ldots \ldots \ldots$

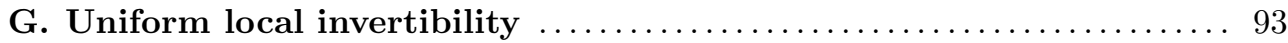

G.1. A sequence adapted to the Picard method $\ldots \ldots \ldots \ldots \ldots \ldots \ldots \ldots$

H. Small initial data on a bounded domain in $\mathbb{R}^{3} \ldots \ldots \ldots \ldots \ldots$

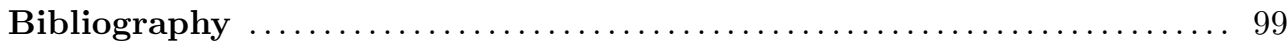




\section{CHAPTER 1}

\section{INTRODUCTION}

In a recent significant paper [30] Corvino has presented a new gluing construction of scalar flat metrics, leading to the striking result of existence of non-trivial scalar flat metrics which are exactly Schwarzschildian at large distances; see also [33]. Extensions of the results in [30] have been announced in [31], and those results should be available ${ }^{(1)}$ in a near future $[\mathbf{3 2}]$. A reading of the proofs in $[\mathbf{3 0}]$ reveals that the arguments there can be simplified or streamlined using known techniques for PDE's in weighted Sobolev spaces $(c f .$, e.g. $[\mathbf{1}, \mathbf{3}, \mathbf{7}, \mathbf{1 6}, \mathbf{4 1}, \mathbf{5 2}])$. Further, the methods introduced by Corvino and Schoen can be applied in other contexts to obtain new classes of solutions of the general relativistic constraint equations. The object of this paper is to present an abstract version, in a large class of weighted Sobolev spaces, of the arguments of Corvino and Schoen. Specific results on compact manifolds with boundary (as considered by Corvino), or on asymptotically flat manifolds, or on asymptotically hyperboloidal manifolds, can then be obtained by an appropriate choice of the weight functions. More precisely, we develop a general theory of mapping properties of the solutions of the linearized constraint operator in a class of weighted Sobolev spaces, assuming certain inequalities. The class of weighted Sobolev spaces includes those of Christodoulou - Choquet-Bruhat [16], appropriate in the asymptotically Euclidean context, as well as an exponentially weighted version thereof, and distance-weighted spaces near a boundary, or an exponentially weighted version thereof; the latter two classes are relevant near a compact boundary, or in an asymptotically hyperboloidal context. We establish the required inequalities in all the spaces just mentioned. An appropriate version of the inverse function theorem allows one to produce new classes of solutions of interest. One application is that of existence of space-times which are Kerrian near spatial infinity; this has already been observed in [31]. We apply our techniques to produce two further large classes of initial data sets with controlled asymptotic behavior at spatial infinity. The first class is obtained by gluing any

${ }^{(1)}$ This paper has been written after $[\mathbf{3 0}, \mathbf{3 1}]$, but independently of $[\mathbf{3 2}]$. 
asymptotically flat initial data with data in the exterior region which are exactly stationary there. This leads to a large class of space-times which are exactly stationary away from the domain of influence of a compact set. The second class consists of initial data which are approximately stationary in the asymptotic region, with the non-stationary part decaying at a prescribed (as high as desired) order in terms of powers of $r$. On the other hand the stationary part is controlled by a set of multipole moments which are freely prescribable within certain ranges. Such initial data are relevant to the program of $[\mathbf{3 7 , 3 9 ]}$. Yet another application is an extension result for initial data near the Minkowskian ones, which leads to asymptotically simple spacetimes, or to new "many black hole" space-times. Our final application here is a gluing construction for generic CMC initial data sets, in which the perturbation of the metric is localized in a small neighborhood of the points where the gluing is performed. This makes use of, and refines, the recent gluing construction of Isenberg, Mazzeo and Pollack $[\mathbf{4 5}, \mathbf{4 6}]$. Some further applications, involving local extensions near positively or negatively curved space forms, or concerning the construction of initial data with controlled Bondi functions, will be discussed elsewhere.

We note that all the results in Section 3 are valid when $M$ is a compact manifold without boundary by setting all the weight functions to one, $\varphi=\phi=\psi=1$, and by taking the compact set $\mathscr{K}$ appearing in Proposition 3.1 and elsewhere equal to $M$.

Acknowledgements. - We thank R. Beig, J. Corvino, H. Friedrich and W. Simon for useful comments or discussions, as well as a referee for detailed criticism. 


\section{CHAPTER 2}

\section{THE CONSTRAINTS MAP}

The aim of this section is to establish some algebraic-differential properties of the constraints map, and some elementary properties of the associated differential operators in a class of weighted Sobolev spaces. The reader is referred to Appendix A for the definition of the latter.

Initial data $(g, K)$ for the vacuum Einstein equations belong to the zero level set of the constraints map:

$$
\left(\begin{array}{l}
J \\
\rho
\end{array}\right)(K, g):=\left(\begin{array}{c}
2\left(-\nabla^{j} K_{i j}+\nabla_{i} \operatorname{tr} K\right) \\
R(g)-|K|^{2}+(\operatorname{tr} K)^{2}
\end{array}\right)=\left(\begin{array}{l}
0 \\
0
\end{array}\right) .
$$

These are the general relativistic constraint equations whatever the space-dimension $n$. As Equations (2.1) are trivial in space-dimension zero and one, in the remainder of this paper we shall assume that $n \geqslant 2$.

Let $h=\delta g$ and $Q=\delta K$, the linearization of the constraints map at $(K, g)$ reads

$$
P(Q, h)=\left(\begin{array}{c}
-K^{p q} \nabla_{i} h_{p q}+K^{q}{ }_{i}\left(2 \nabla^{j} h_{q j}-\nabla_{q} h_{l}{ }_{l}\right) \\
-2 \nabla^{j} Q_{i j}+2 \nabla_{i} \operatorname{tr} Q-2\left(\nabla_{i} K^{p q}-\nabla^{q} K^{p}{ }_{i}\right) h_{p q} \\
-\Delta(\operatorname{tr} h)+\operatorname{div} \operatorname{div} h-\langle h, \operatorname{Ric}(g)\rangle+2 K^{p l} K^{q} h_{l q} \\
-2\langle K, Q\rangle+2 \operatorname{tr} K(-\langle h, K\rangle+\operatorname{tr} Q)
\end{array}\right) .
$$

REMARK 2.1. - We note that for any real numbers $a$ and $b$ it holds

$$
P(a K, b g)=\left(\begin{array}{c}
(a-b) J(K, g) \\
-b R(g)+2(b-a)\left[|K|^{2}-(\operatorname{tr} K)^{2}\right]
\end{array}\right) .
$$

The order of the differential operators that appear in $P$ is

$$
\left(\begin{array}{ll}
1 & 1 \\
0 & 2
\end{array}\right)
$$


which can be written in the Agmon-Douglis-Nirenberg form (cf., e.g. [54, p. 210])

$$
\left(\begin{array}{cc}
s_{1}+t_{1} & s_{1}+t_{2} \\
s_{2}+t_{1} & s_{2}+t_{2}
\end{array}\right)
$$

with $s_{1}=-1, s_{2}=0, t_{1}=t_{2}=2$; here it is understood that an operator of order 0 is also an operator of order 2 with vanishing coefficients in front of the first and second derivatives. It follows that the symbol $P^{\prime}$ of the principal part of $P$ in the sense of Agmon-Douglis-Nirenberg reads

$$
P^{\prime}(x, \xi)(Q, h)=\left(\begin{array}{cc}
2\left(-\xi^{s} \delta_{i}^{t}+\xi_{i} g^{s t}\right) & -K^{p q} \xi_{i}+2 K^{q}{ }_{i} \xi^{p}-K^{l}{ }_{i} \xi_{l} g^{p q} \\
0 & -|\xi|^{2} g^{p q}+\xi^{p} \xi^{q}
\end{array}\right)\left(\begin{array}{c}
Q_{s t} \\
h_{p q}
\end{array}\right),
$$

while the formal $L^{2}$-adjoint of $P$ takes the form

$$
P^{*}(Y, N)=\left(\begin{array}{l}
2\left(\nabla_{(i} Y_{j)}-\nabla^{l} Y_{l} g_{i j}-K_{i j} N+\operatorname{tr} K N g_{i j}\right) \\
\nabla^{l} Y_{l} K_{i j}-2 K^{l}{ }_{(i} \nabla_{j} Y_{l}+K^{q} \nabla_{q} Y^{l} g_{i j}-\Delta N g_{i j}+\nabla_{i} \nabla_{j} N \\
+\left(\nabla^{p} K_{l p} g_{i j}-\nabla_{l} K_{i j}\right) Y^{l}-N \operatorname{Ric}(g)_{i j}+2 N K^{l} K_{j l}-2 N(\operatorname{tr} K) K_{i j}
\end{array}\right) .
$$

From this we obtain the Agmon-Douglis-Nirenberg symbol $P^{* \prime}$ of the principal part of $P^{*}$,

$$
P^{* \prime}(x, \xi)(Y, N)=\left(\begin{array}{cc}
2\left(\xi_{(i} \delta_{j}^{l}-\xi^{l} g_{i j}\right) & 0 \\
K_{i j} \xi^{l}-2 K^{l}{ }_{i} \xi_{j)}+K^{p l} \xi_{q} g_{i j} & \xi_{i} \xi_{j}-|\xi|^{2} g_{i j}
\end{array}\right)\left(\begin{array}{c}
Y_{l} \\
N
\end{array}\right) .
$$

REMARK 2.2. - Recall that the formal adjoint $P^{*}$ is defined by the requirement that for all smooth $(Q, h)$ 's and for all compactly supported smooth $(Y, N)$ 's we have

$$
\left\langle P^{*}(Y, N),(Q, h)\right\rangle_{L^{2}(g) \oplus L^{2}(g)}=\langle(Y, N), P(Q, h)\rangle_{L^{2}(g) \oplus L^{2}(g)} .
$$

It is easily seen by continuity and density arguments that this equation still holds for ${ }^{(1)}$ all $(Q, h) \in H_{\mathrm{loc}}^{1} \times H_{\mathrm{loc}}^{2}$ and for all $(Y, N) \in \stackrel{\circ}{H}_{\phi, \psi}^{1} \times \stackrel{\circ}{H}_{\phi, \psi}^{2}$.

We wish to check ellipticity of $P P^{*}$, for this we need the following:

Lemma 2.3. - Suppose that $\operatorname{dim} M \geqslant 2$, then $P^{* \prime}(x, \xi)$ is injective for $\xi \neq 0$.

Proof. - We define a linear map $\alpha$ from the space $S_{2}$ of two-covariant symmetric tensors into itself by the formula

$$
\alpha(S)=S-(\operatorname{tr} S) g .
$$

Let $\xi \neq 0$, if $(Y, N)$ is in the kernel of $P^{* \prime}(x, \xi)$ then

$$
\alpha\left(\xi_{(i} Y_{j)}\right)=0
$$

so that $\xi_{(i} Y_{j)}=0$, and $Y=0$. It follows that

$$
\alpha\left(\xi_{i} \xi_{j}\right) N=0
$$

which implies $N=0$.

$\overline{{ }^{(1)} \text { See Appendix }}$ A for the definitions of the function spaces we use. 
The lemma implies:

Corollary 2.4. - The operator $L:=P P^{*}$ is elliptic in the sense of AgmonDouglis-Nirenberg (cf., e.g. [54, Definition 6.1.1, p. 210]).

Proof. - The differential order of the various entries of $L$ is

$$
\left(\begin{array}{ll}
2 & 3 \\
3 & 4
\end{array}\right)=\left(\begin{array}{ll}
s_{1}+t_{1} & s_{1}+t_{2} \\
s_{2}+t_{1} & s_{2}+t_{2}
\end{array}\right)
$$

with $s_{1}=-1, s_{2}=0, t_{1}=3, t_{2}=4$. Now, $P^{\prime}(x, \xi)$ is of the form

$$
E:=\left(\begin{array}{cc}
A & B \\
0 & D
\end{array}\right)
$$

while $P^{* \prime}(x, \xi)$ can be written as

$$
\left(\begin{array}{cc}
-{ }^{t} A & 0 \\
-{ }^{t} B & { }^{t} D
\end{array}\right)
$$

where ${ }^{t} X$ denotes the transpose of $X$. Let $\xi \neq 0$; by Lemma $2.3{ }^{t} A$ and ${ }^{t} D$ are injective (hence $A$ and $D$ are surjective), which implies that ${ }^{t} E$ is injective (hence $E$ is surjective). This shows that $E{ }^{t} E$ is bijective: indeed, $E{ }^{t} E X=0$ implies ${ }^{t} X E{ }^{t} E X=0$, which is the same as $\left|{ }^{t} E X\right|^{2}=0$, hence $X=0$. It is straightforward to check that the Agmon-Douglis-Nirenberg symbol of $P P^{*}$, defined as the symbol built from those terms which are precisely of order $s_{i}+t_{j}$, equals

$$
P^{\prime}(x, \xi) P^{* \prime}(x, \xi)=E^{t} E\left(\begin{array}{cc}
-I & 0 \\
0 & 1
\end{array}\right),
$$

and its bijectivity for $\xi \neq 0$ follows. This is precisely the ellipticity condition of Agmon, Douglis, and Nirenberg, whence the result.

We note the following simple fact: ${ }^{(1)}$

Lemma 2.5. - Let $k \in \mathbb{Z}, k \geqslant-2$. Suppose that ${ }^{(2)} g \in W_{\mathrm{loc}}^{k+3, \infty}$ and that

$$
\begin{aligned}
\operatorname{Ric}(g) & \in \phi^{-2} W_{\phi}^{k+2, \infty}, \\
K & \in W_{\phi}^{k+3, \infty} \cap \phi^{-2} W_{\phi}^{k+2, \infty} .
\end{aligned}
$$

If (A.2) holds with $0 \leqslant i \leqslant k+2$, then the linear operators

$$
\begin{aligned}
& P^{*}: \phi \stackrel{\circ}{H}_{\phi, \psi}^{k+3} \times \phi^{2} \stackrel{\circ}{H}_{\phi, \psi}^{k+4} \longrightarrow \stackrel{\circ}{H}_{\phi, \psi}^{k+2} \times \stackrel{\circ}{H}_{\phi, \psi}^{k+2}, \quad k \geqslant-2, \\
& P: \psi^{2}\left(\stackrel{\circ}{H}_{\phi, \psi}^{k+2} \times \stackrel{\circ}{H}_{\phi, \psi}^{k+2}\right) \longrightarrow \psi^{2}\left(\phi^{-1} \stackrel{\circ}{H}_{\phi, \psi}^{k+1} \times \phi^{-2} \stackrel{\circ}{H}_{\phi, \psi}^{k}\right), \quad k \geqslant 0,
\end{aligned}
$$

are well defined, and bounded.

(2) The local differentiability conditions follow from the requirement that the $k+$ fourth covariant derivatives of $N$ and the $k+$ third ones of $Y$ can be defined in a distributional sense; both of those conditions are fulfilled by a metric $g \in W_{\text {loc }}^{k+3, \infty}$ - the reader should note that the first covariant derivatives of $N$ do not involve the Christoffel symbols of $g$ since $N$ is a function. 
Proof. - The result follows immediately from (A.4); we simply mention the inequality

$$
|\phi K| \leqslant \frac{1}{2}\left(\phi^{2}|K|+|K|\right),
$$

which shows that under (2.8) we have $K \in \phi^{-1} W_{\phi}^{k+2, \infty}$; this is used to control the $K^{2}$ terms in $P^{*}$ and in $P$.

We define a map $\Phi$ by

$$
\Phi(x, y):=\left(\phi x, \phi^{2} y\right)
$$

As before, we have the

Lemma 2.6. - Let $k \in \mathbb{Z}$. Suppose that $g \in W_{\mathrm{loc}}^{k+3, \infty}$ and that

$$
\begin{aligned}
\operatorname{Ric}(g) & \in \phi^{-2} W_{\phi}^{k+2, \infty}, \\
K & \in \phi^{-1} W_{\phi}^{k+3, \infty} .
\end{aligned}
$$

If (A.2) holds with $0 \leqslant i \leqslant k+2$, then the linear operators

$$
\begin{aligned}
\Phi P^{*}: \stackrel{\circ}{H}_{\phi, \psi}^{k+3} \times \stackrel{\circ}{H}_{\phi, \psi}^{k+4} \longrightarrow \stackrel{\circ}{H}_{\phi, \psi}^{k+2} \times \stackrel{\circ}{H}_{\phi, \psi}^{k+2}, \quad k \geqslant-2, \\
\psi^{-2} P \Phi \psi^{2}: \stackrel{\circ}{H_{\phi, \psi}^{k+2}} \times \stackrel{\circ}{H}_{\phi, \psi}^{k+2} \longrightarrow \stackrel{\circ}{H}_{\phi, \psi}^{k+1} \times \stackrel{\circ}{H}_{\phi, \psi}^{k}, \quad k \geqslant 0,
\end{aligned}
$$

are well defined, and bounded.

Let us establish now some estimates satisfied by $P^{*}$ :

Lemma 2.7. - Suppose that $g \in W_{\mathrm{loc}}^{1, \infty}$, that (A.2) holds with $0 \leqslant i \leqslant 2$, and that

$$
\begin{aligned}
\operatorname{Ric}(g) & \in \phi^{-2} L^{\infty}, \\
K & \in W_{\phi}^{1, \infty} \cap \phi^{-2} L^{\infty} .
\end{aligned}
$$

Then for any $C^{1}$ vector field $Y$ and $C^{2}$ function $N$, both compactly supported on $\bar{M}$, we have

$$
C\left(\left\|P^{*}\left(\phi Y, \phi^{2} N\right)\right\|_{L_{\psi}^{2}}+\|Y\|_{L_{\psi}^{2}}+\|N\|_{\stackrel{\circ}{\phi}_{\phi, \psi}^{1}}+|b(\phi \psi Y)|^{1 / 2}\right) \geqslant\|Y\|_{\stackrel{\circ}{\phi, \psi}^{1}}+\|N\|_{\stackrel{\circ}{\phi, \psi}^{2},},
$$

where

$$
b(Y)=\int_{\partial M}\left(\nabla_{i} Y_{j} Y^{i}-\nabla_{i} Y^{i} Y_{j}\right) \nu^{j} .
$$

Proof. - Throughout this work the letter $C$ denotes a constant which might change from term to term and line to line. The leading order terms in $P^{*}$ are of the form

$$
\begin{aligned}
P^{*}\left(\phi Y, \phi^{2} N\right)-\text { sub-leading terms } & =:\left(\begin{array}{l}
\widehat{P}_{1}^{*}\left(\phi Y, \phi^{2} N\right) \\
\widehat{P}_{2}^{*}\left(\phi Y, \phi^{2} N\right)
\end{array}\right) \\
& =:\left(\begin{array}{c}
2 \alpha\left(\nabla_{(i}\left(\phi Y_{j}\right)\right. \\
\beta\left(\nabla_{i}\left(\phi Y_{j}\right)\right)+\alpha\left(\nabla_{i} \nabla_{j}\left(\phi^{2} N\right)\right)
\end{array}\right),
\end{aligned}
$$

MÉMOIRES DE LA SMF 94 
and this defines the $\widehat{P}_{1}^{*}, \widehat{P}_{2}^{*}$ and $\beta$ operations (recall that $\alpha$ has been defined in (2.6)). Invertibility of $\alpha$ shows that

$$
\left\|2 \alpha\left(\nabla_{(i} Y_{j}\right)\right\|_{L^{2}} \geqslant C\left\|\nabla_{(i} Y_{j)}\right\|_{L^{2}} .
$$

We have

$$
\int_{M} \nabla_{(i} Y_{j} \nabla^{(i} Y^{j)}=\frac{1}{2}\left(\int_{M} \nabla_{i} Y_{j} \nabla^{i} Y^{j}+\int_{M} \nabla_{i} Y_{j} \nabla^{j} Y^{i}\right),
$$

and Stokes' theorem gives

$$
\int_{M} \nabla_{i} Y_{j} \nabla^{j} Y^{i}=-\int_{M}\left(\nabla^{j} \nabla_{i} Y_{j}\right) Y^{i}+\int_{\partial M}\left(\nabla_{i} Y_{j}\right) Y^{i} \nu^{j}
$$

supposing for the moment that $Y$ is $C^{2}$. Using $\nabla^{j} \nabla_{i} Y_{j}=\nabla_{i} \nabla^{j} Y_{j}+(\operatorname{Ric}(Y, \cdot))_{i}$, and integrating again by parts,

one is led to

$$
-\int_{M} \nabla_{i} \nabla^{j} Y_{j} Y^{i}=\int_{M} \nabla^{j} Y_{j} \nabla_{i} Y^{i}-\int_{\partial M} \nabla^{j} Y_{j} Y^{i} \nu_{i}
$$

$$
\begin{aligned}
\left.\int_{M} \nabla_{(i} Y_{j} \nabla^{(i} Y^{j}\right)=\frac{1}{2}\left(\int_{M}|\nabla Y|^{2}+(\operatorname{div} Y)^{2}\right. & -\operatorname{Ric}(Y, Y) \\
& \left.+\int_{\partial M}\left[\left(\nabla_{i} Y_{j}\right) Y^{i}-\left(\nabla_{i} Y^{i}\right) Y_{j}\right] \nu^{j}\right) .
\end{aligned}
$$

We have thus showed that for $C^{2}$ compactly supported vector fields we have

$$
|b(Y)|+\left\|2 \alpha\left(\nabla_{(i} Y_{j)}\right)\right\|_{L^{2}}+\|\operatorname{Ric}(Y, Y)\|_{L^{1}} \geqslant C\|\nabla Y\|_{L^{2}},
$$

and it should be clear that this remains true for vector fields which are only differentiable once. To continue, we use (2.16) with $Y$ replaced with $\phi \psi Y$; the hypothesis that $\operatorname{Ric}(g) \in \phi^{-2} L_{\phi}^{\infty}$ allows us to write

$$
|b(\phi \psi Y)|+\left\|2 \alpha\left(\nabla_{(i}\left(\phi \psi Y_{j}\right)\right)\right\|_{L^{2}}+\|\psi Y\|_{L^{2}} \geqslant c\|\nabla(\phi \psi Y)\|_{L^{2}} .
$$

We have

$$
\begin{aligned}
& \left\|2 \alpha\left(\nabla_{(i}\left(\phi Y_{j}\right)\right)\right\|_{L_{\psi}^{2}}=\left\|2 \alpha\left(\psi \nabla_{(i}\left(\phi Y_{j)}\right)\right)\right\|_{L^{2}} \\
& \left.=\| 2 \alpha\left(\nabla_{(i}\left(\psi \phi Y_{j)}\right)\right)-2 \alpha\left(\left(\nabla_{(i} \psi\right) \phi Y_{j}\right)\right) \|_{L^{2}} \\
& \geqslant\left\|2 \alpha\left(\nabla_{(i}\left(\phi \psi Y_{j)}\right)\right)\right\|_{L^{2}}-C\left\|\left(\nabla_{(i} \psi\right) \phi Y_{j)}\right\|_{L^{2}} \\
& \geqslant C \|\left(\nabla(\phi \psi Y)\left\|_{L^{2}}-C|b(\phi \psi Y)|-C\right\| \psi Y \|_{L^{2}}\right. \\
& -C\left\|\left(\nabla_{(i} \psi\right) \phi Y_{j}\right\|_{L^{2}} \\
& \geqslant C \|\left(\nabla(\phi \psi) Y+\phi \psi \nabla Y\left\|_{L^{2}}-C|b(\phi \psi Y)|-C\right\| \psi Y \|_{L^{2}}\right. \\
& \left.-C \|\left(\nabla_{(i} \psi\right) \phi Y_{j}\right) \|_{L^{2}} \\
& \geqslant C\|\phi \psi \nabla Y\|_{L^{2}}-C\|\nabla(\phi \psi) Y\|_{L^{2}}-C|b(\phi \psi Y)|-C\|\psi Y\|_{L^{2}} \\
& \left.-C \|\left(\nabla_{(i} \psi\right) \phi Y_{j}\right) \|_{L^{2}}
\end{aligned}
$$

which finally gives

$$
|b(\phi \psi Y)|+\|Y\|_{L_{\psi}^{2}}+\left\|2 \alpha\left(\nabla_{(i}\left(\phi Y_{j}\right)\right)\right\|_{L_{\psi}^{2}} \geqslant C\|\phi \psi \nabla Y\|_{L^{2}} .
$$


Invertibility of $\alpha$ leads us to

$$
\begin{aligned}
\left\|\alpha\left(\nabla \nabla\left(\phi^{2} N\right)\right)\right\|_{L_{\psi}^{2}} & \geqslant C\left\|\nabla \nabla\left(\phi^{2} N\right)\right\|_{L_{\psi}^{2}} \\
& \left.\geqslant C\left\|\phi^{2} \nabla \nabla N\right\|_{L_{\psi}^{2}}-2 C \| \nabla\left(\phi^{2}\right) \nabla N\right)\left\|_{L_{\psi}^{2}}-C\right\| \nabla \nabla\left(\phi^{2}\right) N \|_{L_{\psi}^{2}},
\end{aligned}
$$

so that

$$
\left\|\alpha\left(\nabla \nabla\left(\phi^{2} N\right)\right)\right\|_{L_{\psi}^{2}}+\|N\|_{\dot{H}_{\phi, \psi}^{1}} \geqslant C\left\|\phi^{2} \nabla \nabla N\right\|_{L_{\psi}^{2}} .
$$

Using the hypothesis that $K \in W_{\phi}^{0, \infty}$ we obtain

$$
\begin{aligned}
\left\|\alpha\left(\nabla_{i} \nabla_{j}\left(\phi^{2} N\right)\right)\right\|_{L_{\psi}^{2}} & =\left\|\widehat{P}_{2}^{*}\left(\phi Y, \phi^{2} N\right)-\beta\left(\nabla_{i}\left(\phi Y_{j}\right)\right)\right\|_{L_{\psi}^{2}} \\
& \leqslant\left\|\widehat{P}_{2}^{*}\left(\phi Y, \phi^{2} N\right)\right\|_{L_{\psi}^{2}}+\left\|\beta\left(\nabla_{i}\left(\phi Y_{j}\right)\right)\right\|_{L_{\psi}^{2}} \\
\leqslant & \leqslant \widehat{P}_{2}^{*}\left(\phi Y, \phi^{2} N\right)\left\|_{L_{\psi}^{2}}+C\right\| \nabla_{i}\left(\phi Y_{j}\right) \|_{L_{\psi}^{2}} \\
\leqslant & \left\|\widehat{P}_{2}^{*}\left(\phi Y, \phi^{2} N\right)\right\|_{L_{\psi}^{2}}+C\left\|\widehat{P}_{1}^{*}\left(\phi Y, \phi^{2} N\right)\right\|_{L_{\psi}^{2}} \\
& +C\|Y\|_{L_{\psi}^{2}}+C|b(\phi \psi Y)|,
\end{aligned}
$$

and in the last step we have used (2.18). The lower order terms are controlled using the hypotheses $K \in W_{\phi}^{1, \infty} \cap \phi^{-2} W_{\phi}^{0, \infty}$ and Ric $(g) \in \phi^{-2} W_{\phi}^{0, \infty}$ (compare the proof of Lemma 2.5), leading to (2.14).

We have the following equivalent of Lemma 2.7 for the map considered in Lemma 2.6:

LemmA 2.8. - Suppose that $g \in W_{\mathrm{loc}}^{1, \infty}$, that (A.2) holds with $0 \leqslant i \leqslant 2$, and that

$$
\begin{aligned}
\operatorname{Ric}(g) & \in \phi^{-2} L^{\infty}, \\
K & \in \phi^{-1} W_{\phi}^{1, \infty} .
\end{aligned}
$$

Then for any $C^{1}$ vector field $Y$ and $C^{2}$ function $N$, both compactly supported on $\bar{M}$, we have

$$
C\left(\left\|\Phi P^{*}(Y, N)\right\|_{L_{\psi}^{2}}+\|Y\|_{L_{\psi}^{2}}+\|N\|_{\stackrel{H}{\phi, \psi}_{1}^{1}}+|b(\phi \psi Y)|^{1 / 2}\right) \geqslant\|Y\|_{\stackrel{H}{\phi, \psi}^{1}}+\|N\|_{\dot{H}_{\phi, \psi}^{2}},
$$

where

$$
b(Y)=\int_{\partial M}\left(\nabla_{i} Y_{j} Y^{i}-\nabla_{i} Y^{i} Y_{j}\right) \nu^{j} .
$$

Proof. - The proof is essentially identical with that of Lemma 2.7, with the inequality (2.17) replaced by

$$
\begin{aligned}
\left\|2 \phi \alpha\left(\nabla_{(i} Y_{j}\right)\right\|_{L_{\psi}^{2}} \geqslant C\|\phi \psi \nabla Y\|_{L^{2}}-C \| \nabla(\phi \psi) Y & \|_{L^{2}}-C|b(\phi \psi Y)| \\
& -C\|\psi Y\|_{L^{2}}-C\left\|Y_{(i} \nabla_{j)}(\psi \phi)\right\|_{L^{2}},
\end{aligned}
$$

and inequality (2.19) replaced by

$$
\left\|\phi^{2} \alpha(\nabla \nabla N)\right\|_{L_{\psi}^{2}} \geqslant C\left\|\phi^{2} \nabla \nabla N\right\|_{L_{\psi}^{2}} .
$$

MÉMOIRES DE LA SMF 94 


\section{CHAPTER 3}

\section{ISOMORPHISM THEOREMS}

In this section, we assume that we have a solution $\left(K_{0}, g_{0}\right)$ to the constraint map, with possibly a non-trivial kernel for the associated operator $P_{0}^{*}$, defined as $P^{*}$ with $(K, g)$ replaced by $\left(K_{0}, g_{0}\right)$. We present here a general abstract method to construct "solutions-up-to-kernel" to the constraint equations which are close to $\left(K_{0}, g_{0}\right)$; our argument is a straightforward generalization of [30]. (In particular if the kernel is trivial we obtain solutions.)

Proposition 3.1. - Under the hypotheses of Lemma 2.7 with $(K, g)=\left(K_{0}, g_{0}\right)$, let $\mathcal{K}_{0}$ be the kernel of

$$
P_{0}^{*} \Phi: \stackrel{\circ}{H}_{\phi, \psi}^{1}\left(g_{0}\right) \times \stackrel{\circ}{H}_{\phi, \psi}^{2}\left(g_{0}\right) \longrightarrow L_{\psi}^{2}\left(g_{0}\right) \times L_{\psi}^{2}\left(g_{0}\right),
$$

and let $\mathcal{K}_{0}{ }^{\perp_{g_{0}}}$ be its $L_{\psi}^{2}\left(g_{0}\right) \oplus L_{\psi}^{2}\left(g_{0}\right)$-orthogonal. Assume there exists a compact set $\mathscr{K} \subset M$ such that for all $\stackrel{\circ}{H}_{\phi, \psi}^{1}\left(g_{0}\right)$ vector fields $Y$ and $\stackrel{\circ}{H}_{\phi, \psi}^{2}\left(g_{0}\right)$ functions $N$, both supported in $M \backslash \mathscr{K}$ we have

$$
C\left\|P_{0}^{*} \Phi(Y, N)\right\|_{L_{\psi}^{2}\left(g_{0}\right)} \geqslant\|Y\|_{L_{\psi}^{2}\left(g_{0}\right)}+\|N\|_{\dot{H}_{\phi, \psi}^{1}\left(g_{0}\right)} .
$$

Then there exists a constant $C^{\prime}$ such that

- for all $(K, g)$ close to $\left(K_{0}, g_{0}\right)$ in $\left(W_{\phi}^{1, \infty}\left(g_{0}\right) \cap \phi^{-2} L^{\infty}\left(g_{0}\right)\right) \times W_{\phi}^{2, \infty}\left(g_{0}\right)$ norm,

$$
\text { - and for all }(Y, N) \in \mathcal{K}_{0}^{\perp g} \cap\left(\stackrel{\circ}{H}_{\phi, \psi}^{1}(g) \times \stackrel{\circ}{H}_{\phi, \psi}^{2}(g)\right) \text {, }
$$

it holds that

$$
C^{\prime}\left\|P^{*} \Phi(Y, N)\right\|_{L_{\psi}^{2}(g)} \geqslant\|Y\|_{\dot{H}_{\phi, \psi}^{1}(g)}+\|N\|_{\dot{H}_{\phi, \psi}^{2}(g)} .
$$

REMARK 3.2. - The conclusion still holds if (3.1) is replaced by

$$
C\left(\left\|P^{*}\left(\phi Y, \phi^{2} N\right)\right\|_{L_{\psi}^{2}}+\left\|\left(\phi Y, \phi^{2} N\right)\right\|_{X}\right) \geqslant\|Y\|_{L_{\psi}^{2}}+\|N\|_{H_{\phi, \psi}^{1}},
$$

where $X$ is a normed space such that we have a compact inclusion $\phi \stackrel{\circ}{H}_{\phi, \psi}^{1} \times \phi^{2} \stackrel{\circ}{H}_{\phi, \psi}^{2} \subset X$; however, (3.1) is sufficient for our purposes. 
Proof. - For $(K, g)=\left(K_{0}, g_{0}\right)$, this is proved by a standard argument, compare $[\mathbf{1 , 1 6}]$ : assuming that the inequality fails, there is a sequence

$$
\left(Y_{n}, N_{n}\right) \in\left(\stackrel{\circ}{H}_{\phi, \psi}^{1}\left(g_{0}\right) \times \stackrel{\circ}{H}_{\phi, \psi}^{2}\left(g_{0}\right)\right) \cap \mathcal{K}_{0}{ }_{g_{0}}
$$

with norm 1 such that $\left\|P_{0}^{*} \Phi\left(Y_{n}, N_{n}\right)\right\|_{L_{\psi}^{2}\left(g_{0}\right)}$ approaches zero as $n$ tends to infinity. One obtains a contradiction with injectivity on $\left(\stackrel{\circ}{H}_{\phi, \psi}^{1}\left(g_{0}\right) \times \stackrel{\circ}{H}_{\phi, \psi}^{2}\left(g_{0}\right)\right) \cap \mathcal{K}_{0}{ }^{\perp g_{0}}$ by using the Rellich-Kondrakov compactness on a conditionally compact open set $\mathscr{O} \supset \mathscr{K}$, applying (2.14) with $b(\phi \psi Y)=0$, and (3.1), to $Y$ and $N$ multiplied by suitable cutoff functions; we simply note that (2.14) holds without the boundary term for smooth compactly supported fields ${ }^{(1)}$, hence on $\mathcal{K}_{0}{ }^{\perp_{g_{0}}} \cap\left(\stackrel{\circ}{H}_{\phi, \psi}^{1}\left(g_{0}\right) \times \stackrel{\circ}{H}_{\phi, \psi}^{2}\left(g_{0}\right)\right)$ by density. Increasing $C^{\prime}$ if necessary, the inequality at $\left(K_{0}, g_{0}\right)$ together with straightforward algebra shows that the inequality remains true for $(K, g)$ close to $\left(K_{0}, g_{0}\right)$.

Similarly one obtains:

Proposition 3.3. - Under the hypotheses of Lemma 2.8 with $(K, g)=\left(K_{0}, g_{0}\right)$, let $\mathcal{K}_{0}$ be kernel of

$$
\Phi P_{0}^{*}: \stackrel{\circ}{H}_{\phi, \psi}^{1}\left(g_{0}\right) \times \stackrel{\circ}{H}_{\phi, \psi}^{2}\left(g_{0}\right) \longrightarrow L_{\psi}^{2}\left(g_{0}\right) \times L_{\psi}^{2}\left(g_{0}\right),
$$

and let $\mathcal{K}_{0}{ }^{\perp_{g_{0}}}$ be its $L_{\psi}^{2}\left(g_{0}\right) \oplus L_{\psi}^{2}\left(g_{0}\right)$-orthogonal. Assume there exists a compact set $\mathscr{K} \subset M$ such that for all $\stackrel{\circ}{H}_{\phi, \psi}^{1}\left(g_{0}\right)$ vector fields $Y$ and $\stackrel{\circ}{H}_{\phi, \psi}^{2}\left(g_{0}\right)$ functions $N$, both supported in $M \backslash \mathscr{K}$ we have

$$
C\left\|\Phi P_{0}^{*}(Y, N)\right\|_{L_{\psi}^{2}\left(g_{0}\right)} \geqslant\|Y\|_{L_{\psi}^{2}\left(g_{0}\right)}+\|N\|_{\dot{H}_{\phi, \psi}^{1}\left(g_{0}\right)} .
$$

Then there exists a constant $C^{\prime}$ such that for all $(K, g)$ close to $\left(K_{0}, g_{0}\right)$ in $\phi^{-1} W_{\phi}^{1, \infty}\left(g_{0}\right) \times W_{\phi}^{2, \infty}\left(g_{0}\right)$ norm, and for all $(Y, N) \in \mathcal{K}_{0}^{\perp g} \cap\left(\stackrel{\circ}{H}_{\phi, \psi}^{1}(g) \times \stackrel{\circ}{H}_{\phi, \psi}^{2}(g)\right)$ it holds that

$$
C^{\prime}\left\|\Phi P^{*}(Y, N)\right\|_{L_{\psi}^{2}(g)} \geqslant\|Y\|_{\stackrel{\circ}{\phi, \psi}^{1}(g)}+\|N\|_{\stackrel{\circ}{\phi, \psi}^{2}(g)} .
$$

Set

$$
\mathcal{L}_{\phi, \psi}:=\Phi \psi^{-2} P \psi^{2} P^{*} \Phi .
$$

We denote by $\pi_{\mathcal{K}_{0}^{\perp_{g}}}$ the $L_{\psi}^{2}(g)$ projection onto $\mathcal{K}_{0}^{\perp_{g}}$. We are ready now to prove:

TheOrem 3.4. - Let $k \geqslant 0, g_{0} \in W_{\text {loc }}^{k+4, \infty}$, suppose that (A.2) holds with $0 \leqslant i \leqslant$ $4+k$, and that

$$
\begin{gathered}
\operatorname{Ric}\left(g_{0}\right) \in \phi^{-2} W_{\phi}^{k+2, \infty}\left(g_{0}\right), \\
K_{0} \in W_{\phi}^{k+3, \infty}\left(g_{0}\right) \cap \phi^{-2} W_{\phi}^{k+2, \infty}\left(g_{0}\right) .
\end{gathered}
$$

${ }^{(1)}$ We use the analysts' convention that a manifold $M$ is always open; thus a manifold $M$ with non-empty boundary $\partial M$ does not contain its boundary; instead, $\bar{M}:=M \cup \partial M$ is a manifold with boundary in the differential geometric sense. Unless explicitly specified otherwise no conditions on $M$ are made - e.g. that $\partial M$, if non-empty, is compact - except that $M$ is a smooth manifold; similarly no conditions e.g. on completeness of $(M, g)$, or on its radius of injectivity, are made.

MÉMOIRES DE LA SMF 94 
We further assume that the weights $\phi$ and $\psi$ have the scaling property, cf. the end of Appendix $A$ and Appendix B. If there exists a compact set $\mathscr{K} \subset M$ such that for all $\stackrel{\circ}{H}_{\phi, \psi}^{1}\left(g_{0}\right)$ vector fields $Y$ and $\stackrel{\circ}{H}_{\phi, \psi}^{2}\left(g_{0}\right)$ functions $N$, both supported in $M \backslash \mathscr{K}$, the inequality (3.1) holds, then for all $(K, g)$ close to $\left(K_{0}, g_{0}\right)$ in $\left(W_{\phi}^{k+3, \infty}\left(g_{0}\right) \cap \phi^{-2} W_{\phi}^{k+2, \infty}\left(g_{0}\right)\right) \times W_{\phi}^{k+4, \infty}\left(g_{0}\right)$ norm, the map

$$
\pi_{\mathcal{K}_{0}^{\perp g}} \mathcal{L}_{\phi, \psi}: \mathcal{K}_{0}^{\perp g} \cap\left(\stackrel{\circ}{H}_{\phi, \psi}^{k+3}(g) \times \stackrel{\circ}{H}_{\phi, \psi}^{k+4}(g)\right) \longrightarrow \mathcal{K}_{0}^{\perp g} \cap\left(\stackrel{\circ}{H}_{\phi, \psi}^{k+1}(g) \times \stackrel{\circ}{H}_{\phi, \psi}^{k}(g)\right)
$$

is an isomorphism such that the norm of its inverse is bounded independently ${ }^{(2)}$ of $(K, g)$.

REMARK 3.5. - It is easily seen (see Equation (3.8) below and Remark 2.2) that, in our context, the image of $\mathcal{L}_{\phi, \psi}$ is orthogonal to the kernel of $P^{*} \Phi$. We emphasize, however, that the projection $\pi_{\mathcal{K}_{0}^{\perp_{g}}}$ in (3.6) is on the orthogonal to the kernel of $P_{0}^{*} \Phi$, and not on that of $P^{*} \Phi$.

Proof. - For $(\delta J, \delta \rho) \in \mathcal{K}_{0}^{\perp_{g}} \cap\left(L_{\psi}^{2}(g) \times L_{\psi}^{2}(g)\right)$ let $\mathcal{F}$ be the following (continuous) functional defined on $\mathcal{K}_{0}^{\perp_{g}} \cap\left(\stackrel{\circ}{H}_{\phi, \psi}^{1}(g) \times \stackrel{\circ}{H}_{\phi, \psi}^{2}(g)\right)$ :

$$
\mathcal{F}(Y, N):=\int_{M}\left(\frac{1}{2}\left|P^{*} \Phi(Y, N)\right|_{g}^{2}-\langle(Y, N),(\delta J, \delta \rho)\rangle_{g}\right) \psi^{2} d \mu_{g}
$$

we set

$$
\mu_{F}=\inf _{(Y, N) \in \mathcal{K}_{0}^{\perp g} \cap\left(\stackrel{H}{H}_{\phi, \psi}^{1}(g) \times \stackrel{\circ}{H}_{\phi, \psi}^{2}(g)\right)} \mathcal{F}(Y, N) .
$$

We claim that $\mathcal{F}$ is coercive: indeed, Proposition 3.1 and the Schwarz inequality give

$$
\begin{aligned}
& \mathcal{F}(Y, N) \geqslant C\left(\|Y\|_{\stackrel{\circ}{\phi, ~}^{1}(g)}+\|N\|_{\dot{H}_{\phi, \psi}^{2}(g)}\right)^{2}-\|(Y, N)\|_{L_{\psi}^{2}(g)}\|(\delta J, \delta \rho)\|_{L_{\psi}^{2}(g)} \\
& \geqslant C\left(\|Y\|_{\dot{H}_{\phi, \psi}^{1}(g)}+\|N\|_{\dot{H}_{\phi, \psi}^{2}(g)}\right)^{2}-\left(\|Y\|_{\dot{H}_{\phi, \psi}^{1}(g)}+\|N\|_{\dot{H}_{\phi, \psi}^{2}(g)}\right)\|(\delta J, \delta \rho)\|_{L_{\psi}^{2}(g)} \text {. }
\end{aligned}
$$

Standard results on convex, proper, coercive, l.s.c. (cf., e.g., [36, Proposition 1.2, p. 35]) functionals show that $\mu_{F}$ is achieved by some

$$
(Y, N) \in \mathcal{K}_{0}^{\perp_{g}} \cap\left(\stackrel{\circ}{H}_{\phi, \psi}^{1}(g) \times \stackrel{\circ}{H}_{\phi, \psi}^{2}(g)\right)
$$

satisfying

$$
\begin{aligned}
& \forall(\delta Y, \delta N) \in \stackrel{\circ}{H}_{\phi, \psi}^{1}(g) \times \stackrel{\circ}{H}_{\phi, \psi}^{2}(g) \\
& \left.\qquad \int_{M}\left\langle P^{*} \Phi(Y, N), P^{*} \Phi(\delta Y, \delta N)\right\rangle_{g}-\langle(\delta Y, \delta N),(\delta J, \delta \rho)\rangle_{g}\right) \psi^{2} d \mu_{g}=0 .
\end{aligned}
$$

It follows that $(Y, N) \in \mathcal{K}_{0}^{\perp_{g}} \cap\left(\stackrel{\circ}{H}_{\phi, \psi}^{1}(g) \times \stackrel{\circ}{H}_{\phi, \psi}^{2}(g)\right)$ is a weak solution of the equation

$$
\Phi \psi^{-2} P \psi^{2} P^{*} \Phi(Y, N)=(\delta J, \delta \rho) .
$$

The variational equation (3.7) satisfies the hypotheses of [54, Section 6.4, pp. 242243] with $s_{j}, t_{k}$ as in Corollary 2.4, and with $m_{1}=1, m_{2}=2, h_{0}=-2$. By elliptic

${ }^{(2)}$ The bound on the norm might depend upon $\left(K_{0}, g_{0}\right)$. 
regularity [54, Theorem 6.4.3, p. 246] and by standard scaling arguments ( $c f$. the discussion at the end of Appendix A) for $(\delta J, \delta \rho) \in \stackrel{\circ}{H}_{\phi, \psi}^{k+1}(g) \times \stackrel{\circ}{H}_{\phi, \psi}^{k}(g)$, we have $(Y, N) \in \stackrel{\circ}{H}_{\phi, \psi}^{k+3}(g) \times \stackrel{\circ}{H}_{\phi, \psi}^{k+4}(g)$, and surjectivity follows. To prove bijectivity, we note that the operator $\pi_{\mathcal{K}_{0}^{\perp_{g}}} \mathcal{L}_{\phi, \psi}$ is injective: indeed, if $(Y, N) \in \mathcal{K}_{0}^{\perp_{g}}$ is in the kernel of $\pi_{\mathcal{K}_{0}^{\perp_{g}}} \mathcal{L}_{\phi, \psi}$, then (see Remark 2.2)

$$
0=\left\langle\mathcal{L}_{\phi, \psi}(Y, N),(Y, N)\right\rangle_{L_{\psi}^{2}(g) \oplus L_{\psi}^{2}(g)}=\left\langle P^{*} \Phi(Y, N), P^{*} \Phi(Y, N)\right\rangle_{L_{\psi}^{2}(g) \oplus L_{\psi}^{2}(g)},
$$

so $(Y, N)=0$ from inequality $(3.2)$.

There is yet another operator which is of interest in our context,

$$
L_{\phi, \psi}:=\psi^{-2} P \Phi \psi^{2} \Phi P^{*} .
$$

Similarly to Theorem 3.4, using Proposition 3.3 instead of 3.1, we have:

Theorem 3.6. - Let $k \geqslant 0, g_{0} \in W_{\text {loc }}^{k+4, \infty}$, suppose that (A.2) holds with $0 \leqslant i \leqslant$ $4+k$, that

$$
\begin{aligned}
\operatorname{Ric}\left(g_{0}\right) & \in \phi^{-2} W_{\phi}^{k+2, \infty}\left(g_{0}\right), \\
K_{0} & \in \phi^{-1} W_{\phi}^{k+3, \infty}\left(g_{0}\right),
\end{aligned}
$$

and that the weights $\phi$ and $\psi$ have the scaling property, cf. end of Appendix A. If there exists a compact set $\mathscr{K} \subset M$ such that for all $\stackrel{\circ}{\phi}_{\phi, \psi}^{1}\left(g_{0}\right)$ vector fields $Y$ and $\stackrel{\circ}{H}_{\phi, \psi}^{2}\left(g_{0}\right)$ functions $N$, both supported in $M \backslash \mathscr{K}$, the inequality (3.4) holds, then for all $(K, g)$ close to $\left(K_{0}, g_{0}\right)$ in $\phi^{-1} W_{\phi}^{k+3, \infty}\left(g_{0}\right) \times W_{\phi}^{k+4, \infty}\left(g_{0}\right)$ norm, the map

$$
\pi_{\mathcal{K}_{0}^{\perp_{g}}} L_{\phi, \psi}: \mathcal{K}_{0}^{\perp_{g}} \cap\left(\stackrel{\circ}{H}_{\phi, \psi}^{k+3}(g) \times \stackrel{\circ}{H}_{\phi, \psi}^{k+4}(g)\right) \longrightarrow \mathcal{K}_{0}^{\perp_{g}} \cap\left(\stackrel{\circ}{H}_{\phi, \psi}^{k+1}(g) \times \stackrel{\circ}{H}_{\phi, \psi}^{k}(g)\right)
$$

is an isomorphism such that the norm of its inverse is bounded independently of $(K, g)$.

Whenever the weighted Sobolev spaces are such that the constraints map is defined and differentiable we obtain:

Theorem 3.7. - Under the hypotheses of Theorem 3.4, if the map

$$
\begin{gathered}
\mathcal{K}_{0}^{\perp_{g}} \cap\left(\stackrel{\circ}{H}_{\phi, \psi}^{k+3}(g) \times \stackrel{\circ}{H}_{\phi, \psi}^{k+4}(g)\right) \longrightarrow \mathcal{K}_{0}^{\perp_{g}} \cap\left(\stackrel{\circ}{H}_{\phi, \psi}^{k+1}(g) \times \stackrel{\circ}{H}_{\phi, \psi}^{k}(g)\right) \\
(Y, N) \longmapsto \pi_{\mathcal{K}_{0}^{\perp_{g}}} \psi^{-2} \Phi\left\{\left(\begin{array}{c}
J \\
\rho
\end{array}\right)\left[(K, g)+\psi^{2} P^{*} \Phi(Y, N)\right]-\left(\begin{array}{c}
J \\
\rho
\end{array}\right)(K, g)\right\}
\end{gathered}
$$

is differentiable in a neighborhood $\mathscr{U}_{k}$ of zero, then it is bijective in a (perhaps smaller) neighborhood $\mathscr{V}_{k}$ of zero. In particular there exists $\varepsilon>0$ such that for all $(K, g)$ close to $\left(K_{0}, g_{0}\right)$ in $\left(W_{\phi}^{k+3, \infty}\left(g_{0}\right) \cap \phi^{-2} W_{\phi}^{k+2, \infty}\left(g_{0}\right)\right) \times W_{\phi}^{k+4, \infty}\left(g_{0}\right)$, and for all pairs 
$(\delta J, \delta \rho) \in \psi^{2} \Phi^{-1}\left(\stackrel{\circ}{H}_{\phi, \psi}^{k+1}(g) \times \stackrel{\circ}{H}_{\phi, \psi}^{k}(g)\right)$ with norm less than $\varepsilon$, there exists a solution $(\delta K, \delta g)=\psi^{2} P^{*} \Phi(Y, N) \in \psi^{2}\left(\stackrel{\circ}{H}_{\phi, \psi}^{k+2}(g) \times \stackrel{\circ}{H}_{\phi, \psi}^{k+2}(g)\right)$, close to zero, of the equation

$$
\pi_{\mathcal{K}_{0}^{\perp g}} \psi^{-2} \Phi\left\{\left(\begin{array}{c}
J \\
\rho
\end{array}\right)(K+\delta K, g+\delta g)-\left(\begin{array}{c}
J \\
\rho
\end{array}\right)(K, g)\right\}=\pi_{\mathcal{K}_{0}^{\perp g}} \psi^{-2} \Phi\left(\begin{array}{c}
\delta J \\
\delta \rho
\end{array}\right) .
$$

REMARK 3.8. - The question of differentiability of the map (3.10), or even of its existence, will depend upon the weight functions $\phi$ and $\psi$, and requires a case-by-case treatment.

Proof. - We apply Proposition G.1 with

- $A$ a neighborhood of $\left(K_{0}, g_{0}\right)$ in $\left(W_{\phi}^{k+3, \infty}\left(g_{0}\right) \cap \phi^{-2} W_{\phi}^{k+2, \infty}\left(g_{0}\right)\right) \times W_{\phi}^{k+4, \infty}\left(g_{0}\right)$,

- $x=(K, g), \delta x=(\delta K, \delta g)$,

- $V_{x}=\psi^{2}\left(\stackrel{\circ}{H}_{\phi, \psi}^{k+2}(g) \times \stackrel{\circ}{H}_{\phi, \psi}^{k+2}(g)\right), W_{x}=\mathcal{K}_{0}^{\perp_{g}} \cap\left(\stackrel{\circ}{H}_{\phi, \psi}^{k+1}(g) \times \stackrel{\circ}{H}_{\phi, \psi}^{k}(g)\right)$

- and

$$
f_{x}(\delta x)=\pi_{\mathcal{K}_{0}^{\perp g}} \psi^{-2} \Phi\left\{\left(\begin{array}{c}
J \\
\rho
\end{array}\right)(K+\delta K, g+\delta g)-\left(\begin{array}{c}
J \\
\rho
\end{array}\right)(K, g)\right\} .
$$

We also have the following analogue of Theorem 3.7, with an identical proof, based on Theorem 3.6:

Theorem 3.9. - Under the hypotheses of Theorem 3.6, if the map

$$
\begin{gathered}
\mathcal{K}_{0}^{\perp_{g}} \cap\left(\stackrel{\circ}{H}_{\phi, \psi}^{k+3}(g) \times \stackrel{\circ}{H}_{\phi, \psi}^{k+4}(g)\right) \longrightarrow \mathcal{K}_{0}^{\perp_{g}} \cap\left(\stackrel{\circ}{H}_{\phi, \psi}^{k+1}(g) \times \stackrel{\circ}{H}_{\phi, \psi}^{k}(g)\right) \\
(Y, N) \longmapsto \pi_{\mathcal{K}_{0}^{\perp g}} \psi^{-2}\left\{\left(\begin{array}{c}
J \\
\rho
\end{array}\right)\left[(K, g)+\psi^{2} \Phi^{2} P^{*}(Y, N)\right]-\left(\begin{array}{c}
J \\
\rho
\end{array}\right)(K, g)\right\}
\end{gathered}
$$

is differentiable in a neighborhood of zero, then it is bijective in a (perhaps smaller) neighborhood of zero. Thus, there exists $\varepsilon>0$ such that for all $(K, g)$ close to $\left(K_{0}, g_{0}\right)$ in

$$
\phi^{-1} W_{\phi}^{k+3, \infty}\left(g_{0}\right) \times W_{\phi}^{k+4, \infty}\left(g_{0}\right),
$$

and for all pairs $(\delta J, \delta \rho) \in \psi^{2}\left(\stackrel{\circ}{H}_{\phi, \psi}^{k+1}(g) \times \stackrel{\circ}{H}_{\phi, \psi}^{k}(g)\right)$ with norm less than $\varepsilon$, there exists a solution

$$
(\delta K, \delta g)=\Phi \psi^{2} \Phi P^{*}(Y, N) \in \psi^{2}\left(\phi H_{\phi, \psi}^{k+2}(g) \times \phi^{2} H_{\phi, \psi}^{k+2}(g)\right),
$$

close to zero, of the equation

$$
\pi_{\mathcal{K}_{0}^{\perp g}} \psi^{-2}\left\{\left(\begin{array}{c}
J \\
\rho
\end{array}\right)(K+\delta K, g+\delta g)-\left(\begin{array}{c}
J \\
\rho
\end{array}\right)(K, g)\right\}=\pi_{\mathcal{K}_{0}^{\perp g}} \psi^{-2}\left(\begin{array}{c}
\delta J \\
\delta \rho
\end{array}\right) .
$$

The last results allow us to construct solutions of the nonlinear equation in weighted Sobolev spaces. The drawback of working in such spaces is that the differentiability of the perturbative solutions is considerably worse than that of the starting data $\left(K_{0}, g_{0}\right)$, even when solutions with zero sources are considered. In the usual analysis 
of nonlinear PDE's with implicit-function techniques the higher regularity is obtained by bootstrap arguments. In our set-up this does not work, because the coefficients of the equations do not have enough regularity for the bootstrap. It has been shown by Corvino $[\mathbf{3 0}]$ that there exists a (non-standard) way of getting a partial improvement on the regularity of solutions. This carries over to the general weighted spaces setting considered here provided some further properties of the weights are assumed:

(1) First, note that (A.2) can be rewritten as $\phi \in C_{\phi, \phi^{-1}}^{\ell-1}, \psi \in C_{\phi, \psi^{-1}}^{\ell-1}, \varphi \in C_{\phi, \varphi^{-1}}^{\ell-1}$. When dealing with Hölder spaces one also needs to assume Hölder continuity of the derivatives of the weights, so (renaming $\ell-1$ to $\ell$ ) we will assume:

$$
\phi \in C_{\phi, \phi^{-1}}^{\ell, \alpha}, \quad \psi \in C_{\phi, \psi^{-1}}^{\ell, \alpha}, \quad \varphi \in C_{\phi, \varphi^{-1}}^{\ell, \alpha} .
$$

(2) As discussed in Appendix B, the following conditions are useful for deriving the scaling property: Let us denote by $B_{p}$ the open ball of centre $p$ with radius $\phi(p) / 2$. We assume that there exist constants $C_{1}, C_{2}, C_{3}>0$ such that for all $p \in M$ and all $y \in B_{p}$, we have

$$
\begin{aligned}
& C_{1}^{-1} \phi(p) \leqslant \phi(y) \leqslant C_{1} \phi(p), \\
& C_{2}^{-1} \varphi(p) \leqslant \varphi(y) \leqslant C_{2} \varphi(p), \\
& C_{3}^{-1} \psi(p) \leqslant \psi(y) \leqslant C_{3} \psi(p) .
\end{aligned}
$$

(3) Since the tool to handle non-linearities in this paper is the inverse function theorem, we need to make sure that the changes in the initial data are small as compared to the data themselves. A necessary condition for that is that the new metric be uniformly equivalent to the original one. For example, in the setting of Theorem 3.6, one way of ensuring this is

$$
\psi^{2} \phi^{2} C_{\phi, \varphi}^{k, \alpha}\left(g_{0}\right) \subset C_{\phi, 1}^{k, \alpha}\left(g_{0}\right) .
$$

This will hold under the following condition:

Proposition 3.10. - The inequality

$$
\psi^{2} \phi^{2} \varphi^{-1} \leqslant C
$$

implies (3.19).

In order to check this the reader might wish to prove first that the conditions imposed so far imply that

Lemma 3.11. - If $u \in C_{\phi, \varphi_{1}}^{k, \alpha}(g)$ and $v \in C_{\phi, \varphi_{2}}^{k, \alpha}(g)$, with one of the $\varphi_{a}$ 's satisfying (3.17) and $\phi$ satisfying (3.15) with $\ell \geqslant k$, then $u v \in C_{\phi, \varphi_{1} \varphi_{2}}^{k, \alpha}(g)$.

Lemma 3.11 can be used to show an equivalent of Lemma 2.5 in weighted Hölder spaces.

MÉMOIRES DE LA SMF 94 
(4) The last condition will be the contents of Definition 3.12 that follows. We emphasize that all the conditions spelled out here will be satisfied in all the applications we have in mind.

DEFinition 3.12. - We will say that an operator $L$ from $\stackrel{\circ}{H}_{\phi, \psi}^{3} \times \stackrel{\circ}{H}_{\phi, \psi}^{4}$ to $\stackrel{\circ}{H}_{\phi, \psi}^{1} \times \stackrel{\circ}{H}_{\phi, \psi}^{0}$ satisfies the weighted elliptic regularity condition if there exists a constant $C$ such that for all $(Y, N)$ in $\stackrel{\circ}{H}_{\phi, \psi}^{3} \times \stackrel{\circ}{H}_{\phi, \psi}^{4}$ satisfying $L(Y, N) \in C_{\phi, \varphi}^{k+1, \alpha} \times C_{\phi, \varphi}^{k, \alpha}$ we have $(Y, N) \in$ $C_{\phi, \varphi}^{k+3, \alpha} \times C_{\phi, \varphi}^{k+4, \alpha}$ with

$$
\|(Y, N)\|_{C_{\phi, \varphi}^{k+3, \alpha} \times C_{\phi, \varphi}^{k+4, \alpha}} \leqslant C\left(\|L(Y, N)\|_{C_{\phi, \varphi}^{k+1, \alpha} \times C_{\phi, \varphi}^{k, \alpha}}+\|(Y, N)\|_{H_{\phi, \psi}^{3} \times H_{\phi, \psi}^{4}}\right) .
$$

Armed with those conditions we can pass to an existence theorem in weighted Hölder spaces:

Proposition 3.13 (Existence of solutions in weighted Hölder spaces, I)

Let $k \in \mathbb{N}, 0<\alpha<1$, assume that (3.15) with $\ell \geqslant k+4$ holds, and that (3.16)(3.18) and (3.20) hold, together with

$$
\psi^{2} \varphi^{-1} \leqslant C
$$

In addition to the hypotheses of Theorem 3.7, suppose that $g_{0} \in C^{k+4, \alpha}$, and that

$$
\operatorname{Ric}\left(g_{0}\right) \in \phi^{-2} C_{\phi, 1}^{k+2, \alpha}\left(g_{0}\right), \quad K_{0} \in C_{\phi, 1}^{k+3, \alpha}\left(g_{0}\right) \cap \phi^{-2} C_{\phi, 1}^{k+2, \alpha}\left(g_{0}\right) .
$$

We further assume that the weights $\phi, \varphi$ and $\psi$ have the scaling property, $c f$. the end of Appendix $A$ and Appendix B. Suppose, next, that we have the continuous inclusion

$$
\psi^{2} C_{\phi, \varphi^{2}}^{i, \alpha}(g) \subset \stackrel{\circ}{H}_{\phi, \psi}^{i}(g)
$$

for $i=k, k+1$, with the inclusion norms uniformly bounded for $g$ close to $g_{0}$ in $C_{\phi, 1}^{k+4, \alpha}\left(g_{0}\right)$. Assume finally that $\mathcal{L}_{\phi, \psi}(K, g)$ satisfies the weighted elliptic regularity condition, with a uniform constant $C$ in $(3.21)$ for $(K, g)$ close to $\left(K_{0}, g_{0}\right)$ in

$$
\left(C_{\phi, 1}^{k+3, \alpha}\left(g_{0}\right) \cap \phi^{-2} C_{\phi, 1}^{k+2, \alpha}\left(g_{0}\right)\right) \times C_{\phi, 1}^{k+4, \alpha}\left(g_{0}\right) .
$$

If the source $(\delta J, \delta \rho)$ is in

$$
\psi^{2} \Phi^{-1}\left(\stackrel{\circ}{H}_{\phi, \psi}^{k+1}(g) \times \stackrel{\circ}{H}_{\phi, \psi}^{k}(g)\right) \cap \psi^{2} \Phi^{-1}\left(C_{\phi, \varphi}^{k+1, \alpha}(g) \times C_{\phi, \varphi}^{k, \alpha}(g)\right),
$$

with sufficiently small norm, then the solution obtained in Theorem 3.7 is in

$$
\psi^{2}\left(\stackrel{\circ}{H}_{\phi, \psi}^{k+2}(g) \times \stackrel{\circ}{H}_{\phi, \psi}^{k+2}(g)\right) \cap \psi^{2}\left(C_{\phi, \varphi}^{k+2, \alpha}(g) \times C_{\phi, \varphi}^{k+2, \alpha}(g)\right) .
$$

Proof. - We start with a lemma, which we leave as an exercise to the reader (here Lemma 3.11 together with Equations (3.20) and (3.22) are useful):

LEMMA 3.14. - Under the conditions of Proposition 3.13, the map

$$
\begin{gathered}
C_{\phi, \varphi}^{k+3, \alpha}(g) \times C_{\phi, \varphi}^{k+4, \alpha}(g) \longrightarrow C_{\phi, \varphi}^{k+1, \alpha}(g) \times C_{\phi, \varphi}^{k, \alpha}(g) \\
(Y, N) \longmapsto \psi^{-2} \Phi\left\{\left(\begin{array}{c}
J \\
\rho
\end{array}\right)\left[(K, g)+\psi^{2} P^{*} \Phi(Y, N)\right]-\left(\begin{array}{c}
J \\
\rho
\end{array}\right)(K, g)\right\}
\end{gathered}
$$

is smooth in a neighborhood $\mathscr{U}_{k}$ of zero. 
Returning to the proof of Proposition 3.13, we use the notations of the proof of Theorem 3.7 and we apply Proposition G.2 with $E_{x}=\psi^{2}\left(C_{\phi, \varphi}^{k+2, \alpha}(g) \times C_{\phi, \varphi}^{k+2, \alpha}(g)\right)$, $F_{x}=C_{\phi, \varphi}^{k+1, \alpha}(g) \times C_{\phi, \varphi}^{k, \alpha}(g)$,

$$
G_{x}=\mathcal{K}_{0}^{\perp g} \cap\left(\psi^{2}\left(C_{\phi, \varphi^{2}}^{k+1, \alpha}(g) \times C_{\phi, \varphi^{2}}^{k, \alpha}(g)\right)\right),
$$

and with $A$ - a neighborhood of $\left(K_{0}, g_{0}\right)$ in

$$
\begin{aligned}
{\left[W_{\phi}^{k+3, \infty}\left(g_{0}\right) \cap \phi^{-2} W_{\phi}^{k+2, \infty}\left(g_{0}\right) \times\right.} & \left.W_{\phi}^{k+4, \infty}\left(g_{0}\right)\right] \\
& \cap\left[C_{\phi, 1}^{k+3, \alpha}\left(g_{0}\right) \cap \phi^{-2} C_{\phi, 1}^{k+2, \alpha}\left(g_{0}\right) \times C_{\phi, 1}^{k+4, \alpha}\left(g_{0}\right)\right] .
\end{aligned}
$$

We have continuous inclusions $G_{x} \subset F_{x}$ and $G_{x} \subset W_{x}$ by (3.23). The condition 1 . of Proposition G.2 holds by the hypothesis that $\mathcal{L}_{\phi, \psi}$ satisfies the weighted elliptic regularity condition, and the form of the right inverse used here. Condition 2. and 3. there hold because $J$ and $\rho$ are twice-differentiable (actually smooth) functions of their arguments by Lemma 3.14.

REMARK 3.15. - There is an intriguing mismatch between the order of differentiability of the initial data set $(K, g)$ at which the inverse function theorem is being applied, and the order of differentiability of the final data $(K+\delta K, g+\delta g)$. This seems unavoidable in our setup, and leads to several unpleasant features such as dependence of the neighborhoods on which we can solve the equations upon the degree of differentiability, or failure to produce a Banach manifold structure for the set of solutions, etc. In a forthcoming publication we will give a partial cure to this problem [24].

We continue with Hölder continuous solutions in the setup of Theorem 3.9:

Proposition 3.16 (Existence of solutions in weighted Hölder spaces, II)

Let $k \in \mathbb{N}, 0<\alpha<1$, assume that (3.15) with $\ell \geqslant k+4$ holds, and that (3.16)(3.18) and (3.20) hold. In addition to the hypotheses of Theorem 3.9, suppose that $g_{0} \in C^{k+4, \alpha}$, and that

$$
\operatorname{Ric}\left(g_{0}\right) \in \phi^{-2} C_{\phi, 1}^{k+2, \alpha}\left(g_{0}\right), \quad K_{0} \in \phi^{-1} C_{\phi, 1}^{k+3, \alpha}\left(g_{0}\right) .
$$

We further assume that the weights $\phi, \varphi$ and $\psi$ have the scaling property, $c f$. the end of Appendix A and Appendix B. Suppose, next, that we have the continuous inclusions

$$
\psi^{2} \phi^{2} C_{\phi, \varphi^{2}}^{i, \alpha}(g) \subset \stackrel{\circ}{H}_{\phi, \psi}^{i}(g)
$$

for $i=k, k+1$, with the inclusion norms uniformly bounded for $g$ close to $g_{0}$ in $C_{\phi, 1}^{k+4, \alpha}\left(g_{0}\right)$. Assume finally that $L_{\phi, \psi}(K, g)$ satisfies the weighted elliptic regularity condition, with a uniform constant $C$ in $(3.21)$ for $(K, g)$ close to $\left(K_{0}, g_{0}\right)$ in $\phi^{-1} C_{\phi, 1}^{k+3, \alpha}\left(g_{0}\right) \times C_{\phi, 1}^{k+4, \alpha}\left(g_{0}\right)$. If the source $(\delta J, \delta \rho)$ is in

$$
\psi^{2}\left(\stackrel{\circ}{H}_{\phi, \psi}^{k+1}(g) \times \stackrel{\circ}{H}_{\phi, \psi}^{k}(g)\right) \cap \psi^{2}\left(C_{\phi, \varphi}^{k+1, \alpha}(g) \times C_{\phi, \varphi}^{k, \alpha}(g)\right),
$$

MÉMOIRES DE LA SMF 94 
with sufficiently small norm, then the solution obtained in Theorem 3.9 is in

$$
\psi^{2}\left(\phi \stackrel{\circ}{H}_{\phi, \psi}^{k+2}(g) \times \phi^{2} \stackrel{\circ}{H}_{\phi, \psi}^{k+2}(g)\right) \cap \psi^{2}\left(\phi C_{\phi, \varphi}^{k+2, \alpha}(g) \times \phi^{2} C_{\phi, \varphi}^{k+2, \alpha}(g)\right) .
$$

Propositions 3.13 and 3.16 give existence of Hölder continuous solutions. We can apply the usual bootstrap arguments to those solutions to obtain smoothness, when all the objects at hand are smooth (however, as already pointed out, the bootstrap does not appear to work for solutions in Sobolev spaces):

Proposition 3.17 (Higher regularity). — Let $k \in \mathbb{N}, \alpha \in(0,1)$, assume that (3.15) with $\ell \geqslant k+4$ holds, and that (3.16)-(3.18) and (3.20) hold. Suppose moreover that the scaling property, as spelled out at the end of Appendix A, holds. Assume that $(K, g) \in C^{k+3, \alpha} \times C^{k+4, \alpha}$ and $(Y, N) \in C_{\phi, \varphi}^{3, \alpha}(g) \times C_{\phi, \varphi}^{4, \alpha}(g)$.

(1) If (3.22) holds and if

$$
\left(\begin{array}{l}
J \\
\rho
\end{array}\right)\left[(K, g)+\psi^{2} P^{*} \Phi(Y, N)\right]-\left(\begin{array}{l}
J \\
\rho
\end{array}\right)[(K, g)] \in \psi^{2} \Phi^{-1}\left(C_{\phi, \varphi}^{k+1, \alpha}(g) \times C_{\phi, \varphi}^{k, \alpha}(g)\right),
$$

then $(Y, N) \in C_{\phi, \varphi}^{k+3, \alpha}(g) \times C_{\phi, \varphi}^{k+4, \alpha}(g)$, and thus

$$
(\delta K, \delta g):=\psi^{2} P^{*} \Phi(Y, N) \in \psi^{2}\left(C_{\phi, \varphi}^{k+2, \alpha}(g) \times C_{\phi, \varphi}^{k+2, \alpha}(g)\right) .
$$

(2) Similarly, if

$$
\left(\begin{array}{l}
J \\
\rho
\end{array}\right)\left[(K, g)+\psi^{2} \Phi^{2} P^{*}(Y, N)\right]-\left(\begin{array}{l}
J \\
\rho
\end{array}\right)[(K, g)] \in \psi^{2}\left(C_{\phi, \varphi}^{k+1, \alpha}(g) \times C_{\phi, \varphi}^{k, \alpha}(g)\right),
$$

then $\left.(Y, N) \in C_{\phi, \varphi}^{k+3, \alpha}(g) \times C_{\phi, \varphi}^{k+4, \alpha}(g)\right)$, thus

$$
(\delta K, \delta g) \in \psi^{2}\left(\phi C_{\phi, \varphi}^{k+2, \alpha}(g) \times \phi^{2} C_{\phi, \varphi}^{k+2, \alpha}(g)\right) .
$$

Proof. - It suffices to rewrite the rescaled non-linear elliptic equation (3.27) for $(Y, N)$ as a linear elliptic equation for $(Y, N)$ and freeze coefficients (depending on $(K+\delta K, g+\delta g)$ hence on $(Y, N))$. The interior Hölder estimates [54, Theorem 6.2.5, p. 223] on the sets $\widehat{\Omega}_{\alpha}$ appearing in the definition of scaling property give the local regularity, and the scaling property gives the global weighted regularity.

In situations in which $P^{*}$ has trivial kernel the above theorems produce solutions of the constraint equations. As made clear by the analysis of Corvino [30], solutions can be obtained even when a non-trivial kernel is present in the following circumstances: Suppose that the kernel $\mathcal{K}_{0}$ of $P^{*}$ at $\left(K_{0}, g_{0}\right)$ is non-trivial, set $k=\operatorname{dim} \mathcal{K}_{0}$. Assume we are given a family of pairs $\left(K_{Q, \lambda}, g_{Q, \lambda}\right)$, where $\lambda \in\left[\lambda_{0}, \infty[\right.$ and $Q \in U$, where $U$ is an open in $\mathbb{R}^{k}$, such that $x_{Q, \lambda}:=\left(K_{Q, \lambda}, g_{Q, \lambda}\right)$ goes to $\left(K_{0}, g_{0}\right)$ in $A$ when $\lambda$ goes to infinity, uniformly in $Q \in U$. Assume, in the setup of Theorem 3.7, further that

$$
\psi^{-2} \Phi\left(\begin{array}{c}
\delta J_{Q, \lambda} \\
\delta \rho_{Q, \lambda}
\end{array}\right):=\psi^{-2} \Phi\left\{\left(\begin{array}{l}
J \\
\rho
\end{array}\right)\left(K_{Q, \lambda}, g_{Q, \lambda}\right)-\left(\begin{array}{l}
J \\
\rho
\end{array}\right)\left(K_{0}, g_{0}\right)\right\},
$$

goes to zero in $\stackrel{\circ}{H}_{\phi, \psi}^{k+1}\left(g_{Q, \lambda}\right) \times \stackrel{\circ}{H}_{\phi, \psi}^{k}\left(g_{Q, \lambda}\right)$ when $\lambda$ goes to infinity, uniformly in $Q \in U$. 
If in the setup of Theorem 3.9, assume instead that the same holds for the family

$$
\psi^{-2}\left(\begin{array}{c}
\delta J_{Q, \lambda} \\
\delta \rho_{Q, \lambda}
\end{array}\right):=\psi^{-2}\left\{\left(\begin{array}{c}
J \\
\rho
\end{array}\right)\left(K_{Q, \lambda}, g_{Q, \lambda}\right)-\left(\begin{array}{c}
J \\
\rho
\end{array}\right)\left(K_{0}, g_{0}\right)\right\},
$$

Then for $\lambda$ large enough, $\left(\begin{array}{c}\delta J_{Q, \lambda} \\ \delta \rho_{Q, \lambda}\end{array}\right)$ is less than $\varepsilon$ for all $Q \in U$. So, in the setup of Theorem 3.7, we can solve

$$
f_{x_{Q, \lambda}}\left(\delta x_{Q, \lambda}\right)=-\pi_{\mathcal{K}^{\perp_{Q, \lambda}}} \psi^{-2} \Phi\left(\begin{array}{c}
\delta J_{Q, \lambda} \\
\delta \rho_{Q, \lambda}
\end{array}\right),
$$

while in the setup of Theorem 3.9 we omit the $\Phi$ factor in (3.28); recall that $f_{x}$ has been defined in (3.12). Let $e_{(i)}, i=1, \ldots, k$, be any basis of $\mathcal{K}_{0}$, we define the family of maps

$$
F_{\lambda}: U \longrightarrow \mathbb{R}^{k}
$$

$$
Q \longmapsto\left(\left\langle\psi^{-2} \Phi\left\{\left(\begin{array}{c}
J \\
\rho
\end{array}\right)\left(K_{Q, \lambda}+\delta K_{Q, \lambda}, g_{Q, \lambda}+\delta g_{Q, \lambda}\right)-\left(\begin{array}{c}
J \\
\rho
\end{array}\right)\left(K_{0}, g_{0}\right)\right\}, e_{(i)}\right\rangle_{\mathcal{K}_{0}}\right),
$$

assuming that we are in the context of Theorem 3.7. In the case of Theorem 3.9 the $\Phi$ factor should be removed from (3.29). We note the following result:

LEMmA 3.18. - Let $U$ and $V$ be open sets in $\mathbb{R}^{n}$, suppose that $G$ is a homeomorphism from $U$ to $V$, and consider a family $\left\{G_{\lambda}\right\}_{\lambda \in \mathbb{R}}$ of continuous functions from $U$ to $\mathbb{R}^{n}$ which converge uniformly to $G$ when $\lambda$ goes to infinity. Then for all $y$ in $V$, if $\lambda$ is large enough, there exists $x_{\lambda} \in U$ such that

$$
G_{\lambda}\left(x_{\lambda}\right)=y .
$$

Proof. - Consider the family of maps $u_{\lambda}:=\operatorname{Id}-G_{\lambda} \circ G^{-1}$ from $V$ to $\mathbb{R}^{n}$, the $u_{\lambda}$ 's converge uniformly to 0 when $\lambda$ goes to infinity. Let $y$ in $V$ and let $r>0$ be such that the closed ball $\overline{B(y, r)}$ is included in $V$. If $\lambda$ is large enough, $\sup _{z \in \overline{B(y, r)}}\left|u_{\lambda}(z)\right| \leqslant r$, then the map $z \mapsto y+u_{\lambda}(z)$ is a continuous map from $\overline{B(y, r)}$ to $\overline{B(y, r)}$. From the Brouwer fixed point theorem (cf., e.g. [42]) there exists $y_{\lambda} \in \overline{B(y, r)}$ such that $y_{\lambda}=y+u_{\lambda}\left(y_{\lambda}\right)$, we then set $x_{\lambda}=G^{-1}\left(y_{\lambda}\right)$.

If there exists a function $h(\lambda)$ such that $G_{\lambda}:=h(\lambda) F_{\lambda}$ satisfies the condition of Lemma 3.18, and assuming further that 0 is in $V$, then for all $\lambda$ large enough we can choose $Q_{\lambda}$ such that $F_{\lambda}\left(Q_{\lambda}\right)=0$, hence

$$
\left(\begin{array}{c}
J \\
\rho
\end{array}\right)\left(K_{Q_{\lambda}, \lambda}+\delta K_{Q_{\lambda}, \lambda}, g_{Q_{\lambda}, \lambda}+\delta g_{Q_{\lambda}, \lambda}\right)=\left(\begin{array}{c}
J \\
\rho
\end{array}\right)\left(K_{0}, g_{0}\right) .
$$


It is important to emphasize that if $\left(K_{Q, \lambda}, g_{Q, \lambda}\right)-\left(K_{0}, g_{0}\right)$ is not in $V_{x_{Q, \lambda}}$, then $\left(x_{Q, \lambda}+\delta x_{Q, \lambda}\right) \neq\left(K_{0}, g_{0}\right)$, i.e., we have constructed a solution different from the original one. Summarising, we have shown:

TheOREm 3.19. - Under the hypotheses just described, the projection operators $\pi_{\mathcal{K}_{0}^{\perp g}}$ in (3.11) and (3.14) can be removed for all $\lambda$ large enough. 



\section{CHAPTER 4}

\section{AN ASYMPTOTIC INEQUALITY}

The isomorphism theorems of the previous section all rely on the asymptotic estimate (3.1). The object of this section is to reduce the proof of that estimate to two simpler estimates, one involving only $Y$ and the other involving only $N$. It turns out that some decay conditions are needed for that:

Definition 4.1. - We will say that $(M, g, K, \phi)$ satisfy the asymptotic condition a) if there exists a sequence $U_{i} \subset M$ of open relatively compact sets such that $\bar{U}_{i} \subset U_{i+1}$ (closure in $M$, not in $\bar{M}$ ) with

$$
M=\cup_{i=1}^{\infty} U_{i},
$$

and

$$
\begin{gathered}
\lim _{i \rightarrow \infty}\|K\|_{L^{\infty}\left(M \backslash U_{i}\right)}=\lim _{i \rightarrow \infty}\left\|\phi^{2} K\right\|_{L^{\infty}\left(M \backslash U_{i}\right)}=\lim _{i \rightarrow \infty}\|\phi \nabla K\|_{L^{\infty}\left(M \backslash U_{i}\right)}=0, \\
\lim _{i \rightarrow \infty}\left\|\phi^{2} \operatorname{Ric}(g)\right\|_{L^{\infty}\left(M \backslash U_{i}\right)}=0 .
\end{gathered}
$$

We will say that $(M, g, K, \phi)$ satisfy the asymptotic condition b) if (4.1) and (4.2b) hold and if instead of (4.2a) we have

$$
\lim _{i \rightarrow \infty}\|\phi K\|_{L^{\infty}\left(M \backslash U_{i}\right)}=\lim _{i \rightarrow \infty}\left\|\phi^{2} \nabla K\right\|_{L^{\infty}\left(M \backslash U_{i}\right)}=0,
$$

For any vector field $Y$ set

$$
S(Y)_{i j}:=\nabla_{(i} Y_{j)}=\frac{1}{2}\left(\nabla_{i} Y_{j}+\nabla_{j} Y_{i}\right) .
$$

We can now give a sufficient condition for (3.1):

Lemma 4.2. - Under the hypotheses of Lemma 2.7, assume that $(M, g, K, \phi)$ satisfies the asymptotic condition a). Then (3.1) is equivalent to the requirement that there exists a compact set and a constant $C$ such that for all smooth $(Y, N)$ supported 
outside this compact set we have

$$
\begin{gathered}
C \| S(\phi Y))\left\|_{L_{\psi}^{2}}\left(g_{0}\right) \geqslant\right\| Y \|_{L_{\psi}^{2}}\left(g_{0}\right), \\
C\left\|\nabla \nabla\left(\phi^{2} N\right)\right\|_{L_{\psi}^{2}}\left(g_{0}\right) \geqslant\|N\|_{\stackrel{H}{\phi, \psi}^{1}}\left(g_{0}\right) .
\end{gathered}
$$

Proof. - Setting $Y=0$ or $N=0$ in (3.1) one obtains (4.5) by straightforward manipulations (replacing the compact set $\mathscr{K}$ of Proposition 3.1 by a larger compact set if necessary). In order to prove the reverse implication let us start by establishing the inequality

$$
\|Y\|_{\stackrel{\leftrightarrow}{\phi}_{\phi, \psi}^{1}\left(g_{0}\right)}+\|N\|_{\stackrel{\circ}{\phi, \psi}_{\phi}^{1}\left(g_{0}\right)} \leqslant C\left\|P^{*}\left(\phi Y, \phi^{2} N\right)\right\|_{L_{\psi}^{2}\left(g_{0}\right)},
$$

for all $(Y, N)$ supported in $M \backslash \bar{U}_{i}$, for $i$ large enough. Let $P_{a}^{*}, a=1,2$, be defined as

$$
P^{*}(Y, N)=:\left(\begin{array}{c}
P_{1}^{*}(Y, N) \\
P_{2}^{*}(Y, N)
\end{array}\right)
$$

from Equation (2.4) one finds

$$
\left\|-2 \alpha(S(\phi Y))+P_{1}^{*}\left(\phi Y, \phi^{2} N\right)\right\|_{L_{\psi}^{2}\left(g_{0}\right)} \leqslant C\left\|\phi^{2} K\right\|_{L^{\infty}}\|N\|_{L_{\psi}^{2}\left(g_{0}\right)},
$$

where $\alpha$ is as in (2.15). Equation (4.5a) together with Equation (2.18) (with $b=0$ there) yield

$$
\begin{aligned}
c\|Y\|_{\dot{H}_{\phi, \psi}^{1}\left(g_{0}\right)} & \leqslant 2\|Y\|_{L_{\psi}^{2}\left(g_{0}\right)}+2\|\alpha(S(\phi Y))\|_{L_{\psi}^{2}\left(g_{0}\right)} \\
& \leqslant C\|S(\phi Y)\|_{L_{\psi}^{2}\left(g_{0}\right)}+2\|\alpha(S(\phi Y))\|_{L_{\psi}^{2}\left(g_{0}\right)} \leqslant C^{\prime}\|\alpha(S(\phi Y))\|_{L_{\psi}^{2}\left(g_{0}\right)} \\
& =C^{\prime}\left\|\alpha(S(\phi Y))+P_{1}^{*}\left(\phi Y, \phi^{2} N\right)-P_{1}^{*}\left(\phi Y, \phi^{2} N\right)\right\|_{L_{\psi}^{2}\left(g_{0}\right)} \\
& \leqslant C^{\prime}\left(\left\|P_{1}^{*}\left(\phi Y, \phi^{2} N\right)\right\|_{L_{\psi}^{2}\left(g_{0}\right)}+C\left\|\phi^{2} K\right\|_{L^{\infty}}\|N\|_{L_{\psi}^{2}\left(g_{0}\right)}\right) .
\end{aligned}
$$

Applying (4.5b) it holds that

$$
\|N\|_{\hat{H}_{\phi, \psi}^{1}\left(g_{0}\right)} \leqslant C\left\|\nabla \nabla\left(\phi^{2} N\right)\right\|_{L_{\psi}^{2}\left(g_{0}\right)} \leqslant C^{2} \| \alpha\left(\nabla \nabla\left(\phi^{2} N\right) \|_{L_{\psi}^{2}\left(g_{0}\right)} .\right.
$$

From Equation (2.4) we have

$$
\begin{aligned}
& \left\|\alpha\left(\nabla \nabla\left(\phi^{2} N\right)\right)-P_{2}^{*}\left(\phi Y, \phi^{2} N\right)\right\|_{L_{\psi}^{2}\left(g_{0}\right)} \\
& \leqslant C\left(\|K\|_{L^{\infty}\left(M \backslash U_{i}\right)}\|\nabla(\phi Y)\|_{L_{\psi}^{2}\left(g_{0}\right)}+\|\phi \nabla K\|_{L^{\infty}\left(M \backslash U_{i}\right)}\|Y\|_{L_{\psi}^{2}\left(g_{0}\right)}\right. \\
& \left.\quad+\left(\left\|\phi^{2} \operatorname{Ric}\right\|_{L^{\infty}\left(M \backslash U_{i}\right)}+\left\|(\phi K)^{2}\right\|_{L^{\infty}\left(M \backslash U_{i}\right)}\right)\|N\|_{L_{\psi}^{2}\left(g_{0}\right)}\right) \\
& \leqslant \varepsilon\left(\|Y\|_{\dot{H}_{\phi, \psi}^{1}\left(g_{0}\right)}+\|N\|_{L_{\psi}^{2}\left(g_{0}\right)}\right),
\end{aligned}
$$

where $\varepsilon$ can be made as small as desired by choosing $i$ large enough. It then follows from Equation (4.9) that

$$
\|N\|_{\stackrel{H}{\phi, \psi}^{1}\left(g_{0}\right)} \leqslant C\left\|P_{2}^{*}\left(\phi Y, \phi^{2} N\right)\right\|_{L_{\psi}^{2}\left(g_{0}\right)}+C \varepsilon\left(\|Y\|_{\stackrel{H}{\phi, \psi}^{1}\left(g_{0}\right)}+\|N\|_{L_{\psi}^{2}\left(g_{0}\right)}\right) .
$$


Adding (4.8) and (4.11), and choosing $i$ large enough — so that $\varepsilon$ is small enough one obtains Equation (4.6). We note that from (4.6) by similar manipulations one can further obtain

$$
\|Y\|_{\stackrel{\leftrightarrow}{\phi, ~}_{\phi}^{1}\left(g_{0}\right)}+\|N\|_{\stackrel{\leftrightarrow}{\phi, \psi}^{2}\left(g_{0}\right)} \leqslant C\left\|P^{*}\left(\phi Y, \phi^{2} N\right)\right\|_{L_{\psi}^{2}\left(g_{0}\right)},
$$

but this is irrelevant for our purposes.

An identical calculation yields:

Lemma 4.3. - Under the hypotheses of Lemma 2.8, assume that $(M, g, K, \phi)$ satisfies the asymptotic condition b). Then (3.4) is equivalent to the requirement that for all smooth $(Y, N)$ supported outside a compact set we have

$$
\begin{gathered}
C \| \phi S(Y))\left\|_{L_{\psi}^{2}}\left(g_{0}\right) \geqslant\right\| Y \|_{L_{\psi}^{2}}\left(g_{0}\right), \\
C\left\|\phi^{2} \nabla \nabla N\right\|_{L_{\psi}^{2}}\left(g_{0}\right) \geqslant\|N\|_{\stackrel{H}{\phi, \psi}^{1}}\left(g_{0}\right) .
\end{gathered}
$$





\section{CHAPTER 5}

\section{COMPACT BOUNDARIES}

Let us justify the inequality (4.5a) in a neighborhood of a compact boundary $\partial M$. We assume that the metric is as in Appendix C, in particular Equation (C.3) holds. We start with the following:

Proposition 5.1. - Let $s \neq-1 / 2$ and suppose that

$$
|\operatorname{Hess}(x)|=o\left(x^{-1}\right) \text {. }
$$

Then there exists a neighborhood $\mathcal{O}_{s}$ of $\partial M$ such that for every $C^{1}$ vector field with compact support in $\mathcal{O}_{s} \backslash \partial M$ we have

$$
\int x^{2 s}|Y|^{2} \leqslant C \int x^{2 s+2}|S(Y)|^{2}
$$

for some constant $C$, where $S$ is defined by Equation (4.4).

REMARK 5.2. - The restriction $s \neq-1 / 2$ is sharp, which can be seen by considering the family of vector fields $\chi_{n}\left(1-\chi_{n_{0}}\right) Y, n \geqslant n_{0}$, where $Y$ is a Killing vector which does not vanish on $\partial M$, and where the cut-off functions $\chi_{n}$ are defined as $\chi_{n}(x)=1$ for $x \geqslant 1 / n, \chi_{n}(x)=\ln (2 n x) / \ln 2$ for $1 /(2 n) \leqslant x \leqslant 1 / n, \chi_{n}(x)=0$ otherwise. The resulting $\chi_{n}$ 's are not $C^{1}$, but this is enough to invalidate (5.1); in any case, a small smooth perturbation of $\chi_{n}$ will yield the required $C^{1}$ example.

Proof. - The result is a straightforward consequence of Corollary D.4.

We shall consider metrics which can be quite singular near the boundary; this is mainly motivated by the applications to conformally compactifiable metrics, see Section 6 below. To control the boundary behavior of $g$ we thus introduce the following definition:

Definition 5.3. - Let $k \in \mathbb{N}$ and let $\mathcal{W}$ be a space of symmetric tensors on $M$. We shall say that $g$ has an $(\mathcal{W}, k)$-behavior at $\partial M$ if there exists a metric $g_{\bar{M}}$ on $\bar{M}$ of class $C^{k}(\bar{M})$ such that $g-g_{\bar{M}} \in \mathcal{W}$. 
In the remainder of this section we assume that $\bar{M}$ is a compact manifold with boundary. We take the weight function $\phi$ as

$$
\phi:=x,
$$

where $x$ is any defining function for $\partial M$. For $k \in \mathbb{N}$ and $s \in \mathbb{R}$, we define

$$
\stackrel{\circ}{\mathrm{H}}_{k}^{s}(g):=\stackrel{\circ}{H}_{x, x^{-s-n / 2}}^{k}(g) \text {. }
$$

The labeling of the spaces here is motivated by the following decay property ( $c f$., e.g., $[\mathbf{1}])$

$$
f \in \stackrel{\circ}{\mathrm{H}}_{k}^{\beta}(g), k>n / 2 \quad \Longrightarrow \quad f=o\left(x^{\beta}\right)
$$

We also have

$$
x^{\sigma} \in \stackrel{\circ}{H}_{k}^{s}(g) \text { iff } \sigma>s+(n-1) / 2 .
$$

Let us define in the same way

$$
\mathrm{C}_{k, \alpha}^{s}(g):=C_{x, x^{-s}}^{k, \alpha}(g)
$$

When studying boundary behavior of solutions of PDE's near boundaries, alternative useful classes of weighted spaces are obtained as follows: in a collar neighborhood of $\partial M$ one introduces coordinate systems $\left(x, v^{A}\right)$, with $\partial M$ being given by the equation $\{x=0\}$. Instead of adding a weight factor $x$ for each derivative, one adds $x$ factors to the $\partial_{x}$ derivatives only. Functions in such weighted spaces have more tangential regularity, as compared with functions in the $\mathrm{H}$ spaces or $\mathrm{C}$ spaces. However, some of the simple scaling arguments which we have been using so far do not apply, and considerably more work is required (see, e.g., $[\mathbf{3}]$ ) to obtain a priori estimates in such spaces. While those alternative spaces could probably be used in our context here, leading to solutions with higher regularity, we have not attempted to carry through a systematic study.

We start with the following:

THEOREM 5.4. - Let $\bar{M}$ be a compact manifold with boundary, let $k \geqslant 0$, and suppose that $g_{0}$ is a metric on $M$ which has $\left(W_{x}^{k+4, \infty}, k+4\right)$-behavior at $\partial M$, with

$$
\begin{gathered}
x^{2}\left|\operatorname{Ric}\left(g_{0}\right)\right|_{g_{0}} \longrightarrow{ }_{x \rightarrow 0} 0, \\
x|\nabla \nabla x|_{g_{0}} \longrightarrow{ }_{x \rightarrow 0} 0, \\
K_{0} \in x^{-1} W_{x}^{k+3, \infty}\left(g_{0}\right), \quad x\left|K_{0}\right|_{g_{0}}+x^{2}\left|\nabla K_{0}\right|_{g_{0}} \longrightarrow{ }_{x \rightarrow 0} 0 .
\end{gathered}
$$

Then for all $s \neq(n-1) / 2,(n-3) / 2$ and all $(K, g)$ close to $\left(K_{0}, g_{0}\right)$ in $x^{-1} W_{x}^{k+3, \infty}\left(g_{0}\right) \times$ $W_{x}^{k+4, \infty}\left(g_{0}\right)$ norm, the map

$$
\left.\pi_{\mathcal{K}_{0}^{\perp g}} L_{x, x^{s-n / 2}}: \mathcal{K}_{0}^{\perp_{g}} \cap\left(\stackrel{\circ}{\mathrm{H}}_{k+3}^{-s}(g) \times{\stackrel{\circ}{\mathrm{H}_{k+4}^{-s}}}^{-s}\right)\right) \longrightarrow \mathcal{K}_{0}^{\perp_{g}} \cap\left(\circ_{\mathrm{H}}^{-s}(g) \times \stackrel{\circ}{\mathrm{H}}_{k}^{-s}(g)\right)
$$

is an isomorphism such that the norm of its inverse is bounded independently of $(K, g)$.

Remark 5.5. - Conditions (5.4a)-(5.4b) will hold if there exists $\alpha>0$ such that $g$ has $\left(x^{\alpha} W_{x}^{k+4, \infty}, k+4\right)$-behavior at $\partial M$. 
Proof. - We wish to apply Theorem 3.6, in order to do that we need to establish the inequality (3.4) for $Y$ 's and $N$ 's supported outside of a sufficiently a large ball. For $s \neq(n-1) / 2$, Proposition 5.1 yields

$$
\|Y\|_{\mathrm{H}_{0}^{-s}} \leqslant C\|x S(Y)\|_{\mathrm{H}_{0}^{-s}} .
$$

Applying Proposition C.3 twice we find that for $s \neq(n-1) / 2,(n-3) / 2$ it holds that

$$
\|N\|_{\stackrel{\mathrm{H}}{0}_{0}^{-s}} \leqslant C\|x \nabla N\|_{\stackrel{\circ}{0}_{0}^{-s}} \leqslant C^{2}\left\|x^{2} \nabla \nabla N\right\|_{\stackrel{\mathrm{H}}{0}^{-s}} \leqslant C^{3}\left\|x^{2} \alpha(\nabla \nabla N)\right\|_{\mathrm{H}_{0}^{-s}},
$$

where $\alpha$ is as in (2.6). Now $(M, g, K, x)$ satisfy the asymptotic condition $b)$ of Definition 4.1 with $U_{i}=\{x>1 / i\}$, and Lemma 4.3 shows that we can apply Theorem 3.6.

Our first main application of the abstract results of the previous sections is surjectivity up to kernel of $P^{*}$ of the constraint map. In particular surjectivity is obtained if no kernel is present; a case with kernel will be analyzed in Section 8.1.

TheOREm 5.6. - Under the hypotheses of Theorem 5.4 with $s \geqslant n-2, s>1$ if $n=3, k>n / 2$, the map

$$
\begin{aligned}
& \mathcal{K}_{0}^{\perp_{g}} \cap\left(\stackrel{\circ}{\mathrm{H}}_{k+3}^{-s}(g) \times \stackrel{\circ}{\mathrm{H}}_{k+4}^{-s}(g)\right) \longrightarrow \mathcal{K}_{0}^{\perp g} \cap\left(\stackrel{\circ}{\mathrm{H}}_{k+1}^{-s}(g) \times \stackrel{\circ}{\mathrm{H}}_{k}^{-s}(g)\right) \\
& (Y, N) \longmapsto \pi_{\mathcal{K}_{0}^{\perp_{g}}} x^{-2 s+n}\left\{\left(\begin{array}{c}
J \\
\rho
\end{array}\right)\left[(K, g)+x^{2 s-n} \Phi^{2} P^{*}(Y, N)\right]-\left(\begin{array}{c}
J \\
\rho
\end{array}\right)(K, g)\right\}
\end{aligned}
$$

is bijective in a neighborhood of zero. More precisely, there exists $\varepsilon>0$ such that for all $(K, g)$ in $x^{-1} W_{x}^{k+3, \infty}\left(g_{0}\right) \times W_{x}^{k+4, \infty}\left(g_{0}\right)$ for which

$$
\left\|\left(K-K_{0}, g-g_{0}\right)\right\|_{\left.x^{-1} W_{x}^{k+3, \infty}\left(g_{0}\right)\right) \times W_{x}^{k+4, \infty}\left(g_{0}\right)}<\varepsilon
$$

and for all pairs $(\delta J, \delta \rho) \in \stackrel{\circ}{\mathrm{H}}_{k+1}^{s-n}(g) \times \stackrel{\circ}{\mathrm{H}}_{k}^{s-n}(g)$ satisfying

$$
\|(\delta J, \delta \rho)\|_{\dot{H}_{k+1}^{s-n}(g) \times \dot{H}_{k}^{s-n}(g)}<\varepsilon
$$

there exists a solution $\left.(\delta K, \delta g)=x^{2 s-n} \Phi^{2} P^{*}(Y, N) \in{\stackrel{\circ}{\mathrm{H}^{2}}}_{k+2}^{s-n+1}(g) \times \stackrel{\circ}{\mathrm{H}}_{k+2}^{s-n+2}(g)\right)$, close to zero, of the equation

$$
\pi_{\mathcal{K}_{0}^{\perp g}} x^{-2 s+n}\left\{\left(\begin{array}{c}
J \\
\rho
\end{array}\right)(K+\delta K, g+\delta g)-\left(\begin{array}{c}
J \\
\rho
\end{array}\right)(K, g)\right\}=\pi_{\mathcal{K}_{0}^{\perp g}} x^{-2 s+n}\left(\begin{array}{c}
\delta J \\
\delta \rho
\end{array}\right) .
$$

Proof. - The conditions $s \geqslant n-1$ and $k>n / 2$ ensure that the map of Equation (5.7) is well defined and differentiable in a neighborhood of zero; a relatively straightforward though lengthy check of that can be done using weighted Moser inequalities (see [26] for proofs in a slightly different context; the arguments there adapt to the current setting in a straightforward way). The result follows then from Theorem 3.9.

We also have solutions with Hölder regularity: 
Proposition 5.7. - Let $\bar{M}$ be a compact manifold with boundary, let $k \geqslant k_{0}:=$ $\lfloor n / 2\rfloor+1$ (the smallest integer strictly larger than $n / 2), \alpha \in(0,1)$, and suppose that $g_{0}$ is a metric on $M$ which has $\left(\mathrm{C}_{k+4, \alpha}^{0}, k+5\right)$-behavior at $\partial M$, with

$$
\begin{gathered}
x^{2}\left|\operatorname{Ric}\left(g_{0}\right)\right|_{g_{0}} \longrightarrow{ }_{x \rightarrow 0} 0, \\
x|\nabla \nabla x|_{g_{0}} \longrightarrow{ }_{x \rightarrow 0} 0 \\
K_{0} \in \mathrm{C}_{k+3, \alpha}^{-1}\left(g_{0}\right), \quad x\left|K_{0}\right|_{g_{0}}+x^{2}\left|\nabla K_{0}\right|_{g_{0}} \longrightarrow{ }_{x \rightarrow 0} 0 .
\end{gathered}
$$

There exists $\varepsilon>0$ such that if $(K, g)$ in $\mathrm{C}_{k+3, \alpha}^{-1}\left(g_{0}\right) \times \mathrm{C}_{k+4, \alpha}^{0}\left(g_{0}\right)$, and if

$$
\begin{array}{r}
\left\|\left(K-K_{0}, g-g_{0}\right)\right\|_{x^{-1} W_{x}^{k_{0}+3, \infty}\left(g_{0}\right) \times W_{x}^{k_{0}+4, \infty}\left(g_{0}\right)}<\varepsilon \\
\|(\delta J, \delta \rho)\|_{\mathrm{C}_{k_{0}+1, \alpha}^{t}(g) \times \mathrm{C}_{k_{0}, \alpha}^{t}(g)}+\|(\delta J, \delta \rho)\|_{\dot{H}_{k_{0}+1}^{t}(g) \times \dot{\mathrm{H}}_{k_{0}}^{t}(g)}<\varepsilon
\end{array}
$$

for some $t \geqslant-2, t>-2$ if $n=3$, then the solution $(\delta K, \delta g)$ given by Theorem 5.6 (with $s=t+n)$ is in $\mathrm{C}_{k_{0}+2, \alpha}^{t+1}(g) \times \mathrm{C}_{k_{0}+2, \alpha}^{t+2}(g)$. If moreover

$$
(\delta J, \delta \rho) \in \mathrm{C}_{k+1, \alpha}^{t}(g) \times \mathrm{C}_{k, \alpha}^{t}(g)
$$

then the solution given by Theorem 5.6 is in $\mathrm{C}_{k+2, \alpha}^{t+1}(g) \times \mathrm{C}_{k+2, \alpha}^{t+2}(g)$.

Remark 5.8. - All the hypotheses in Proposition 5.7 will hold if $g_{0}$ has $\left(\mathrm{C}_{k+4, \alpha}^{\beta}, k+5\right)$ behavior at $\partial M$, for some $\beta>0$, with $K_{0} \in \mathrm{C}_{k+3, \alpha}^{\beta-1}$; in particular they will hold if $\left(K_{0}, g_{0}\right) \in C^{k+4}(\bar{M}) \times C^{k+5}(\bar{M})$.

Proof. - Under the current hypotheses all the conditions of Proposition 3.16 with $k$ there equal to $k_{0}$ are met. (The weighted elliptic regularity condition of Definition 3.12 is satisfied by the calculation (B.4), Appendix B.) The higher Hölder regularity follows from Proposition 3.17.

A useful class of solutions is obtained by taking the weight to decay exponentially at the boundary: the weighting functions are then chosen to be $\phi=x^{2}$ and $\psi=e^{s / x}$. The main interest of this class of spaces stems from the inclusion

$$
\cap_{k \in \mathbb{N}} C_{x^{2}, e^{s / x}}^{k+\alpha} \subset C^{\infty}(\bar{M}),
$$

which holds on a compact manifold with boundary $\bar{M}$ for any $s>0$. Here the space $C^{\infty}(\bar{M})$ denotes the space of tensor fields which extend smoothly to $\partial M$, together with all their derivatives; in fact all fields belonging to the left-hand-side of the inclusion above can be smoothly extended by a zero tensor field. It is shown at the end of Appendix B that the spaces $H_{x^{2}, e^{s / x}}^{k}$ satisfy the hypotheses of Lemma B.1; the latter asserts that the scaling property holds for those spaces. This gives:

Theorem 5.9. - Let $\bar{M}$ be a compact manifold with boundary, let $s>0, k>n / 2$ and suppose that $g_{0}$ is a metric on $M$ which has $\left(W_{x^{2}}^{k+4, \infty}, k+4\right)$-behavior at $\partial M$, 
with

$$
\begin{gathered}
x^{4}\left|\operatorname{Ric}\left(g_{0}\right)\right|_{g_{0}} \longrightarrow{ }_{x \rightarrow 0} 0, \\
x|\nabla \nabla x|_{g_{0}} \longrightarrow{ }_{x \rightarrow 0} 0, \\
K_{0} \in x^{-2} W_{x^{2}}^{k+3, \infty}\left(g_{0}\right), \quad x^{2}\left|K_{0}\right|_{g_{0}}+x^{4}\left|\nabla K_{0}\right|_{g_{0}} \longrightarrow{ }_{x \rightarrow 0} 0 .
\end{gathered}
$$

There exists $\varepsilon>0$ such that for all $(K, g)$ in $x^{-2} W_{x^{2}}^{k+3, \infty}\left(g_{0}\right) \times W_{x^{2}}^{k+4, \infty}\left(g_{0}\right)$ for which

$$
\left\|\left(K-K_{0}, g-g_{0}\right)\right\|_{x^{-2} W_{x^{2}}^{k+3, \infty}\left(g_{0}\right) \times W_{x^{2}}^{k+4, \infty}\left(g_{0}\right)}<\varepsilon,
$$

and for all pairs $(\delta J, \delta \rho) \in H_{x^{2}, e^{s / x}}^{k+1}(g) \times H_{x^{2}, e^{s / x}}^{k}(g)$ satisfying

$$
\|(\delta J, \delta \rho)\|_{H_{x^{2}, e^{s / x}}^{k+1}(g) \times H_{x^{2}, e^{s / x}}^{k}(g)}<\varepsilon
$$

there exists a solution $\left.(\delta K, \delta g)=e^{-2 s / x} \Phi^{2} P^{*}(Y, N) \in x^{2} H_{x^{2}, e^{s / x}}^{k+2}(g) \times x^{4} H_{x^{2}, e^{s / x}}^{k+2}(g)\right)$, close to zero, of the equation

$$
\pi_{\mathcal{K}_{0}^{\perp g}} e^{2 s / x}\left\{\left(\begin{array}{c}
J \\
\rho
\end{array}\right)(K+\delta K, g+\delta g)-\left(\begin{array}{c}
J \\
\rho
\end{array}\right)(K, g)\right\}=\pi_{\mathcal{K}_{0}^{\perp g}} e^{2 s / x}\left(\begin{array}{c}
\delta J \\
\delta \rho
\end{array}\right) .
$$

Proof. - As before, we apply Theorems 3.6 and 3.9. We first show the inequality (3.4) for $Y$ 's and $N$ 's supported in a sufficiently small neighborhood of $\partial M$. For $s \neq 0$, consider the equality in Corollary D.5. Taking absolute values of both sides, and applying Cauchy-Schwarz to the left term in the resulting equality one obtains

$$
\|Y\|_{H_{x^{2}, e^{s / x}}^{0}} \leqslant C\left\|x^{2} S(Y)\right\|_{H_{x^{2}, e^{s / x}}^{0}} .
$$

Applying Proposition C.4 with $u:=x^{2} \nabla N$ and with $u=N$, we find that for $s \neq 0$ it holds that

$$
\begin{aligned}
\left\|x^{4} \nabla \nabla N\right\|_{H_{x^{2}, e^{s / x}}^{0}} & \geqslant C_{1}\left\|x^{2} \nabla N\right\|_{H_{x^{2}, e^{s / x}}^{0}} \\
& =\frac{C_{1}}{2}\left\|x^{2} \nabla N\right\|_{H_{x^{2}, e^{s / x}}^{0}}+\frac{C_{1}}{2}\left\|x^{2} \nabla N\right\|_{H_{x^{2}, e^{s / x}}^{0}} \\
& \geqslant \frac{C_{1}}{2}\left\|x^{2} \nabla N\right\|_{H_{x^{2}, e^{s / x}}^{0}}+C_{2}\|N\|_{H_{x^{2}, e^{s / x}}^{0}} \\
& \geqslant C_{3}\|N\|_{H_{x^{2}, e^{s / x}}^{1}} .
\end{aligned}
$$

The inequality (3.4) is then satisfied. Now $(M, g, K, x)$ satisfy the asymptotic condition b) of Definition 4.1 with $U_{i}=\{x>1 / i\}$, and Lemma 4.3 shows that we can apply Theorem 3.6. The conditions $s>0$ and $k>n / 2$ ensure that the map of Equation (3.13) is well defined and differentiable in a neighborhood of zero. (Here one should use weighted Moser inequalities, which can be established by the methods of $[\mathbf{2 6}]$ together with the scaling arguments of Appendix B.) The result follows then from Theorem 3.9. 
It is easy to check that the spaces $H_{x^{2}, e^{s / x}}^{k}$ in Theorem 5.9 can be replaced by the spaces $H_{x^{2}, x^{2 a} e^{s / x}}^{k}$, for any $a \in \mathbb{R}$, we leave the details to the reader. The need for such a generalization arises when wishing to pass from weighted Sobolev spaces to exponentially weighted Hölder spaces: indeed, Equation (B.4), Appendix B, gives

$$
\|u\|_{C_{x^{2}, x^{2 a} e^{s / x}}^{k+m, \alpha}} \leqslant C\left(\|P u\|_{C_{x^{2}, x^{2 a} e^{s / x}}^{k, \alpha}(M)}+\|u\|_{L_{x^{2(a-n / 2)} e^{s / x}}^{2(M)}}\right) .
$$

and leads to the following proposition, the details are left to the reader:

Proposition 5.10. - Let $\bar{M}$ be a compact manifold with boundary, let $k \geqslant k_{0}:=$ $\lfloor n / 2\rfloor+1$ (the smallest integer strictly larger than $n / 2$ ), and suppose that $g_{0}$ is a metric on $M$ which has $\left(C_{x^{2}, 1}^{k+4, \alpha}, k+5\right)$-behavior at $\partial M$. Assume that (5.10) holds. Then there exists $\varepsilon>0$ such that if $(K, g)$ in $x^{-2} C_{x^{2}, 1}^{k+3, \alpha}\left(g_{0}\right) \times C_{x^{2}, 1}^{k+4, \alpha}\left(g_{0}\right)$ with

$$
\left\|\left(K-K_{0}, g-g_{0}\right)\right\|_{x^{-2} W_{x^{2}}^{k_{0}+3, \infty}\left(g_{0}\right) \times W_{x^{2}}^{k_{0}+4, \infty}\left(g_{0}\right)}<\varepsilon,
$$

and if

$$
\|(\delta J, \delta \rho)\|_{C_{x^{2}, e^{t / x}}^{k_{0}+1, \alpha}(g) \times C_{x^{2}, e^{t / x}}^{k_{0}, \alpha}}+\|(\delta J, \delta \rho)\|_{H_{x^{2}, x^{-n} e^{t / x}}^{k_{0}+1}(g) \times H_{x^{2}, x^{-n} e^{t / x}}^{k_{0}}(g)}<\varepsilon
$$

with $t>0$, then the solution $(\delta K, \delta g)$ given by Theorem 5.9 is in $x^{2} C_{x^{2}, e^{t / x}}^{k_{0}+2, \alpha}(g) \times$ $x^{4} C_{x^{2}, e^{t / x}}^{k_{0}+2, \alpha}(g)$. If moreover $(\delta J, \delta \rho) \in C_{x^{2}, e^{t / x}}^{k+1, \alpha}(g) \times C_{x^{2}, e^{t / x}}^{k, \alpha}(g)$ then the solution given by Theorem 5.9 is in $x^{2} C_{x^{2}, e^{t / x}}^{k+2, \alpha}(g) \times x^{4} C_{x^{2}, e^{t / x}}^{k+2, \alpha}(g)$.

Choose some $\alpha>0$ and define the Fréchet spaces $C_{x^{2}, e^{s / x}}^{\infty}(g)$ as the collection of all functions or tensor fields which are in $C_{x^{2}, e^{s / x}}^{k, \alpha}(g)$ whatever $k \in \mathbb{N}$, equipped with the family of semi-norms $\left\{\|\cdot\|_{C_{x^{2}, e^{s / x}}^{k, \alpha}}, k \in \mathbb{N}\right\}$. We then have:

CoROllary 5.11. - Under the hypotheses of the preceding proposition, if $(\delta J, \delta \rho) \in$ $C_{x^{2}, e^{s / x}}^{\infty}(g) \times C_{x^{2}, e^{s / x}}^{\infty}(g)$, and if $(K, g) \in C^{\infty}(\bar{M}) \times C^{\infty}(\bar{M})$, then the solution given by Theorem 5.9 is in

$$
x^{2} C_{x^{2}, e^{s / x}}^{\infty}(g) \times x^{4} C_{x^{2}, e^{s / x}}^{\infty}(g) \subset C^{\infty}(\bar{M}) \times C^{\infty}(\bar{M}) .
$$

In fact $(\delta K, \delta g)$ can be smoothly extended by zero across $\partial M$. 


\section{CHAPTER 6}

\section{CONFORMALLY COMPACTIFIABLE INITIAL DATA}

A Riemannian manifold $(M, g)$ will be said to be conformally compactifiable if

(1) $\bar{M}=M \cup \partial M$ is a compact manifold with non-empty boundary;

(2) let $x$ be any defining function for $\partial M$, then the tensor field $x^{2} g$ extends by continuity to a continuous Riemannian metric $\bar{g}$ on $\bar{M}$.

This definition encompasses Riemannian manifolds such as hyperbolic space. The associated initial data occur in the context of space-times which are asymptotically flat in lightlike directions $[\mathbf{3}, \mathbf{4}, \mathbf{3 9}]$, or in that of asymptotically anti-de Sitter spacetimes $[\mathbf{3 8}, \mathbf{4 8}]$.

The topological setup here is thus identical to that of the previous section, but the metrics $g$ differ from the ones used there by a rescaling factor $x^{2}$. It turns out that there is a simple correspondence of the functional spaces $\stackrel{\mathrm{H}}{k}_{k}^{\alpha}$ from the previous section with a class of natural weighted spaces associated to conformally compactifiable metrics: since $g=x^{-2} \bar{g}$ on $M$ we obviously have

$$
L_{\psi}^{2}(g)=L_{x^{-n / 2} \psi}^{2}(\bar{g}) .
$$

Further, assuming that (A.2) holds for $0 \leqslant i \leqslant k$ with $\phi=x$ and $g$ there replaced by $\bar{g}$ (recall that this will hold if $\bar{g}$ has $\left(W_{x}^{k, \infty}, k\right)$-behavior at $\partial M$ in the sense of Definition 5.3, in particular that will be the case if $\bar{g}$ is $C^{k}(\bar{M})$ ) it is simple to check that

$$
H_{1, \psi}^{k}(g)=H_{x, x^{-n / 2} \psi}^{k}(\bar{g})
$$

for tensors; $0 \leqslant i \leqslant k-1$ in (A.2) would suffice for functions. It is therefore natural (see (5.2)-(5.3)) to define

$$
\stackrel{\circ}{\mathcal{H}}_{k}^{s}(g):=\stackrel{\circ}{H}_{1, x^{-s}}^{k}(g)=\stackrel{\circ}{\mathrm{H}}_{k}^{s}(\bar{g}) .
$$

Let us define in the same way

$$
\mathcal{C}_{k, \alpha}^{s}(g):=C_{x, x^{-s}}^{k, \alpha}(\bar{g})=\mathrm{C}_{k, \alpha}^{s}(\bar{g})
$$


We note that

$$
x^{\sigma} \in \mathcal{H}_{k}^{s} \quad \text { iff } \sigma>s+(n-1) / 2
$$

Similarly to (5.3) we have

$$
f \in \mathcal{H}_{k}^{\beta}(g), k>n / 2 \quad \Longrightarrow \quad f=o\left(x^{\beta}\right) .
$$

We will be mainly interested in conformally compactifiable metrics such that $x^{2} g$ has $\left(\mathcal{H}_{k}^{\alpha}, k\right)$ - or $\mathcal{C}_{k, \alpha}^{s}$-behavior at the conformal boundary, $\alpha>0$; such metrics arise naturally when solving the constraint equations via the conformal method $[\mathbf{3}, \mathbf{4}]$.

We will need some estimates on $P^{*}(Y, N)$ extending those of Section 4 , when

$$
K=\lambda g+L,
$$

where $\lambda$ is a uniformly bounded function on $M$. We will further assume that

$$
|L|_{\bar{g}}=o\left(x^{-2}\right) \text { and }|\nabla L|_{\bar{g}}=o\left(x^{-3}\right) .
$$

If we use this particular choice of $K$ in equation (2.4), we find

$$
\begin{aligned}
P^{*}(Y, N)=\left(\begin{array}{c}
P_{1}^{*}(Y, N) \\
P_{2}^{*}(Y, N)
\end{array}\right) \\
\quad=\left(\begin{array}{l}
2\left(\nabla_{(i} Y_{j}-\nabla^{l} Y_{l} g_{i j}-g_{i j} \lambda N+n \lambda N g_{i j}\right)+o\left(x^{-2}\right) N \\
2 \lambda\left(\nabla^{l} Y_{l} g_{i j}-\nabla_{(i} Y_{j)}\right)-\Delta N g_{i j}+\nabla_{i} \nabla_{j} N-N \operatorname{Ric}(g)_{i j} \\
+2 N \lambda^{2} g_{i j}-2 N n \lambda^{2} g_{i j}+o\left(x^{-2}\right)(\nabla Y)+o\left(x^{-3}\right)(Y)+o\left(x^{-2}\right) N
\end{array}\right),
\end{aligned}
$$

where $o\left(x^{\alpha}\right)$ denotes a tensor the $\bar{g}$-norm of which is $o\left(x^{\alpha}\right)$. We then have

$$
\begin{aligned}
\lambda P_{1}^{*}(Y, N)+P_{2}^{*}(Y, N)=-\Delta N g_{i j} & +\nabla_{i} \nabla_{j} N-N \operatorname{Ric}(g)_{i j} \\
& +o\left(x^{-2}\right)(\nabla Y)+o\left(x^{-3}\right)(Y)+o\left(x^{-2}\right) N .
\end{aligned}
$$

If we prove that for all $(Y, N)$ supported in a neighborhood $\mathcal{O}$ of $\partial M$,

$$
\begin{gathered}
\|S(Y)\|_{\mathcal{H}_{0}^{s}} \geqslant C\|Y\|_{\mathcal{H}_{0}^{s}}, \\
\|-\Delta N g+\nabla \nabla N-N \operatorname{Ric}(g)\|_{\mathcal{H}_{0}^{s}} \geqslant C\|N\|_{\mathcal{H}_{1}^{s}} .
\end{gathered}
$$

then we will have from (6.5a) and (2.18):

$$
\|Y\|_{\mathcal{H}_{1}^{\circ}} \leqslant C\left(\|Y\|_{\mathcal{H}_{0}^{s}}+\|S(Y)\|_{\mathcal{H}_{0}^{s}}\right) \leqslant C^{\prime}\|S(Y)\|_{\mathcal{H}_{0}^{\circ}} \leqslant C^{\prime \prime}\left(\left\|P_{1}^{*}(Y, N)\right\|_{\mathcal{H}_{0}^{\circ}}+\|N\|_{\mathcal{H}_{0}^{s}}\right),
$$

and from (6.5b) and (6.4),

$$
\|\lambda\|_{L^{\infty}}\left\|P_{1}^{*}(Y, N)\right\|_{\mathcal{H}_{0}^{s}}+\left\|P_{2}^{*}(Y, N)\right\|_{\mathcal{H}_{0}^{s}} \geqslant C\|N\|_{\mathcal{H}_{1}^{s}}-\varepsilon\|Y\|_{\mathcal{H}_{1}^{s}},
$$

where $\varepsilon$ is arbitrary close to zero, reducing $\mathcal{O}$ if necessary. Finally $\varepsilon(6.6)+(6.7)$ gives

$$
\left(\|\lambda\|_{L^{\infty}}+1\right)\left\|P_{1}^{*}(Y, N)\right\|_{\mathcal{H}_{0}^{s}}+\left\|P_{2}^{*}(Y, N)\right\|_{\mathcal{H}_{0}^{s}} \geqslant C\|N\|_{\mathcal{H}_{1}^{s}},
$$

then for $\varepsilon$ small, we obtain the asymptotic inequality (3.4) (with $\Phi=$ id) for $P^{*}$ :

$$
\left(\|\lambda\|_{L^{\infty}}+1\right)\left\|P_{1}^{*}(Y, N)\right\|_{\mathcal{H}_{0}^{s}}+\left\|P_{2}^{*}(Y, N)\right\|_{\mathcal{H}_{0}^{s}} \geqslant C\|Y\|_{\mathcal{H}_{0}^{s}}+C\|N\|_{\mathcal{H}_{1}^{s}} .
$$


Let us justify the inequality (6.5a) in a neighborhood of a compact boundary $\partial M$. We have the following:

Proposition 6.1. - Let $s \neq(n-1) / 2,(n+1) / 2$. Then there exists a neighborhood $\mathcal{O}_{s}$ of $\partial M$ such that for every $C^{1}$ vector field with compact support in $\mathcal{O}_{s} \backslash \partial M$ we have

$$
\int x^{2 s}|Y|^{2} d \mu_{g} \leqslant C \int x^{2 s}|S(Y)|^{2} d \mu_{g},
$$

for some constant $C$, where $S$ is defined by Equation (4.4).

REMARK 6.2. - The argument given in Remark 5.2 shows that the restriction $s \neq$ $(n+1) / 2$ is sharp. We suspect that the restriction $s \neq(n-1) / 2$ can be removed.

Proof. - This is just a rewriting of Corollary D.12.

THEOREM 6.3. - Let $\bar{M}$ be a compact manifold with boundary, let $k \geqslant 0$, and suppose that $g_{0}$ is a conformally compact metric on $M$ such that $g_{0}=x^{-2} \bar{g}_{0}$, with $\bar{g}_{0}$ having $\left(W_{x}^{k+4, \infty}, k+4\right)$-behavior at $\partial M$. Assume that

(6.9) $K_{0}=\lambda_{0} g_{0}+L_{0}, \quad L_{0}, \lambda_{0} \in W_{1}^{k+3, \infty}\left(g_{0}\right),\left|L_{0}\right|_{g_{0}} \longrightarrow{ }_{x \rightarrow 0} 0, \quad\left|\nabla L_{0}\right|_{g_{0}} \longrightarrow{ }_{x \rightarrow 0} 0$.

Then for all $s \neq(n-3) / 2,(n-1) / 2,(n+1) / 2$ and all $(K, g)$ close to $\left(K_{0}, g_{0}\right)$ in the $W_{1}^{k+3, \infty}\left(g_{0}\right) \times W_{1}^{k+4, \infty}\left(g_{0}\right)$ norm, the map

$$
\pi_{\mathcal{K}_{0}^{\perp g}} L_{1, x^{s}}: \mathcal{K}_{0}^{\perp_{g}} \cap\left(\stackrel{\circ}{\mathrm{H}}_{k+3}^{-s}(g) \times \stackrel{\circ}{\mathrm{H}}_{k+4}^{-s}(g)\right) \longrightarrow \mathcal{K}_{0}^{\perp_{g}} \cap\left(\stackrel{\circ}{\mathrm{H}}_{k+1}^{-s}(g) \times \stackrel{\circ}{\mathrm{H}}_{k}^{-s}(g)\right)
$$

is an isomorphism such that the norm of its inverse does not depend upon $(K, g)$.

Proof. - We wish to apply Theorem 3.6. It follows from the discussion above that we only need to establish the inequality (6.5) for $Y$ 's and $N$ 's supported outside of a sufficiently a large compact set. For $s \neq(n-1) / 2,(n+1) / 2$, Proposition 6.1 yields

$$
\|Y\|_{\mathrm{H}_{0}^{-s}} \leqslant C\|S(Y)\|_{\mathrm{H}_{0}^{-s}} .
$$

Applying Proposition D.13 we find that for $s \neq(n-3) / 2,(n-1) / 2,(n+1) / 2$ it holds that

$$
\|N\|_{\mathrm{H}_{1}^{-s}} \leqslant C\|-\Delta N g+\nabla \nabla N-N \operatorname{Ric}(g)\|_{\mathrm{H}_{0}^{-s}},
$$

which is what had to be established.

A proof identical to that of Theorem 5.6 yields:

THEOREM 6.4. - Under the hypotheses of Theorem 6.3 with

$$
s \geqslant 0, \quad s \neq(n-3) / 2,(n-1) / 2,(n+1) / 2, \quad k>n / 2,
$$


the map

$$
\begin{gathered}
\mathcal{K}_{0}{ }^{\perp_{g}} \cap\left(\stackrel{\circ}{\mathrm{H}}_{k+3}^{-s}(g) \times \stackrel{\circ}{\mathrm{H}}_{k+4}^{-s}(g)\right) \longrightarrow \mathcal{K}_{0}{ }^{\perp_{g}} \cap\left(\stackrel{\circ}{\mathrm{H}}_{k+1}^{-s}(g) \times \stackrel{\circ}{\mathrm{H}}_{k}^{-s}(g)\right) \\
(Y, N) \longmapsto \pi_{\mathcal{K}_{0} \perp_{g}} x^{-2 s}\left\{\left(\begin{array}{c}
J \\
\rho
\end{array}\right)\left[(K, g)+x^{2 s} P^{*}(Y, N)\right]-\left(\begin{array}{c}
J \\
\rho
\end{array}\right)(K, g)\right\}
\end{gathered}
$$

is bijective in a neighborhood of zero. Thus, there exists $\varepsilon>0$ such that for all $(K, g)$ close to $\left(K_{0}, g_{0}\right)$ in $W_{1}^{k+3, \infty}\left(g_{0}\right) \times W_{1}^{k+4, \infty}\left(g_{0}\right)$, and for all pairs $(\delta J, \delta \rho) \in \stackrel{\circ}{\mathrm{H}}_{k+1}^{s}(g) \times$ $\stackrel{\circ}{\mathrm{H}}_{k}^{s}(g)$ with norm less than $\varepsilon$, there exists a solution $(\delta K, \delta g)=x^{2 s} P^{*}(Y, N) \in$ $\left.\stackrel{\circ}{\mathrm{H}}_{k+2}^{s}(g) \times \stackrel{\circ}{\mathrm{H}}_{k+2}^{s}(g)\right)$, close to zero, of the equation

$$
\pi_{\mathcal{K}_{0}{ }^{\perp_{g}}} x^{-2 s}\left\{\left(\begin{array}{c}
J \\
\rho
\end{array}\right)(K+\delta K, g+\delta g)-\left(\begin{array}{c}
J \\
\rho
\end{array}\right)(K, g)\right\}=\pi_{\mathcal{K}_{0}{ }^{{ }_{g}}} x^{-2 s}\left(\begin{array}{c}
\delta J \\
\delta \rho
\end{array}\right) .
$$

REMARK 6.5. - For metrics which are sufficiently regular at the conformal boundary it can be shown that any non-trivial solution of the equation $P^{*}(Y, N)=0$ satisfies $|N|+|Y|_{g} \sim 1 / x$ near $x=0$. This shows that if $0 \leqslant s<(n+1) / 2$, then $\mathcal{K}_{0}=\{0\}$, so that no projection operator is necessary in (6.13).

Proof. - The conditions $s \geqslant 0$ and $k>n / 2$ ensure that the map of Equation (6.12) is well defined and differentiable in a neighborhood of zero; a relatively straightforward though lengthy check of that can be done using weighted Moser inequalities (see [26] for proofs in a slightly different context; the arguments there adapt to the current setting in a straightforward way). The solvability of the equation (6.13) follows then from Theorem 3.9.

A proof identical to that of Proposition 5.7 gives solutions with Hölder regularity: Proposition 6.6. - Let $\bar{M}$ be a compact manifold with boundary, let $k \geqslant k_{0}:=$ $\lfloor n / 2\rfloor+1$ (the smallest integer strictly larger than $n / 2), \alpha \in(0,1)$, and suppose that $g_{0}$ is a conformally compact metric on $M$ such that $g_{0}=x^{-2} \bar{g}_{0}$, with $\bar{g}_{0}$ having $\left(\mathcal{C}_{k+4, \alpha}^{0}, k+5\right)$-behavior at $\partial M$. Assume that

(6.14) $K_{0}=\lambda_{0} g_{0}+L_{0}, \quad L_{0}, \lambda_{0} \in \mathcal{C}_{k+3, \alpha}^{0}\left(g_{0}\right),\left|L_{0}\right|_{g_{0}} \longrightarrow{ }_{x \rightarrow 0} 0, \quad\left|\nabla L_{0}\right|_{g_{0}} \longrightarrow{ }_{x \rightarrow 0} 0$.

Then for all $t \geqslant 0, t \neq(n-3) / 2,(n-1) / 2,(n+1) / 2$ there exists $\varepsilon>0$ such that if $(K, g)$ in $\mathcal{C}_{k+3, \alpha}^{0}\left(g_{0}\right) \times \mathcal{C}_{k+4, \alpha}^{0}\left(g_{0}\right)$, and if

$$
\begin{aligned}
& \left\|\left(K-K_{0}, g-g_{0}\right)\right\|_{W_{1}^{k_{0}+3, \infty}\left(g_{0}\right) \times W_{1}^{k_{0}+4, \infty}\left(g_{0}\right)}<\varepsilon \\
& \|(\delta J, \delta \rho)\|_{\mathcal{C}_{k_{0}+1, \alpha}^{t}(g) \times \mathcal{C}_{k_{0}, \alpha}^{t}(g)}+\|(\delta J, \delta \rho)\|_{{\stackrel{\mathrm{H}}{k_{0}+1}}^{t}}(g) \times{\stackrel{\circ}{\mathrm{H}_{k_{0}}^{t}}}^{(g)}<\varepsilon,
\end{aligned}
$$

then the solution $(\delta K, \delta g)$ given by Theorem 6.4 (with $s=t$ ) is in $\mathcal{C}_{k_{0}+2, \alpha}^{t}(g) \times$ $\mathcal{C}_{k_{0}+2, \alpha}^{t}(g)$. If moreover $(\delta J, \delta \rho) \in \mathcal{C}_{k+1, \alpha}^{t}(g) \times \mathcal{C}_{k, \alpha}^{t}(g)$ then the solution given by Theorem 6.4 is in $\mathcal{C}_{k+2, \alpha}^{t}(g) \times \mathcal{C}_{k+2, \alpha}^{t}(g)$.

One has very similar results in exponentially weighted Hölder and Sobolev spaces as at the end of Section 5 , the details are left to the reader.

MÉMOIRES DE LA SMF 94 


\section{CHAPTER 7}

\section{ASYMPTOTICALLY FLAT INITIAL DATA}

Definition 7.1. - Let $W$ be a space of symmetric tensors on $\mathbb{R}^{n} \backslash \overline{B\left(R_{0}\right)}, R_{0} \geqslant$ 1 , where $B\left(R_{0}\right)$ is an open coordinate ball of radius $R_{0}$ in $\mathbb{R}^{n}$. We shall say that $(M, g)$ is $W$-asymptotically flat if there exists a set $\mathscr{K} \subset M$ and a diffeomorphism $\chi^{-1}: M \backslash \mathscr{K} \rightarrow \mathbb{R}^{n} \backslash \overline{B\left(R_{0}\right)}$ such that

$$
\left(\chi^{*} g\right)_{i j}-\delta_{i j} \in W .
$$

The region $M_{\text {ext }}:=M \backslash \mathscr{K}$ will be called an end of $M$. $M$ will be said to have compact interior if $\mathscr{K}$ is compact.

In the above definition we have assumed that $M$ has only one end, there is an obvious natural generalization of the above notion to any finite number of ends; the results below generalize without any difficulties to such cases. We emphasize that in Definition 7.1 the manifold $M$ is allowed to have a compact boundary. We will often use the symbol $r$ to denote a function $f$ on $M$ such that $f \circ \chi$ coincides with the radius $r$ on $\mathbb{R}^{n} \backslash B(R)$ for $R$ large enough. The requirement $R_{0} \geqslant 1$ has been made for notational convenience, to guarantee that the function $r$, which will be used as a weight on $M_{\text {ext }}$, is strictly positive there.

The simplest choice for the $W$ spaces above are the $C_{k}^{\alpha}$ Hölder spaces, defined as the spaces of functions such that

$$
\|f\|_{C_{k}^{\alpha}}:=\left\|\left(1+r^{2}\right)^{-\alpha / 2} f\right\|_{C_{k}^{0}}<\infty,
$$

where $\|\cdot\|_{C_{k}^{0}}$ is the sum of sup norms of $f$ and its derivatives up to order $k$, with each derivative entering with a supplementary factor of $r$. If

$$
f=f_{j_{1} \ldots j_{\ell}}^{i_{1} \ldots i_{i_{1}}} \otimes \cdots \otimes \partial_{i_{k}} \otimes d x^{j_{1}} \otimes \cdots \otimes d x^{j_{\ell}}
$$

is a tensor field, then (7.1) should be used for each entry $f^{i_{1} \ldots i_{k}}{ }_{j_{1} \ldots j_{\ell}}$ of $f$, with respect to the natural coordinates $x^{i}$ on $\mathbb{R}^{n}$, and a sum over the $i_{p}$ 's and $j_{q}$ 's of the norms $\left\|f^{i_{1} \ldots i_{k}}{ }_{j_{1} \ldots j_{\ell}}\right\|_{C_{k}^{\alpha}}$ should be made. 
Somewhat sharper results can be obtained when working with manifolds for which $W$ is a weighted Sobolev space. We shall say that $f \in r^{\alpha} W_{r}^{k, \infty}$ if $\left(1+r^{2}\right)^{-\alpha / 2} f \in$ $W_{r}^{k, \infty}$. This is equivalent to the requirement that $f \in C_{k-1}^{\alpha}$, and that the distributional $k$ 'th derivatives of $f$ satisfy a weighted Lipschitz condition. The metrics, solutions of the constraint equations which are obtained by our methods, are $\left(r^{-\alpha} W_{r}^{k+2, \infty}+\stackrel{\circ}{H}_{r, r^{-\sigma}}^{k}\right)$-asymptotically flat with some $\alpha, \sigma>0, k \geqslant 2$.

It is convenient to relabel the $\stackrel{\circ}{H}_{r, r^{\alpha}}^{k}$ spaces as follows: for $k \in \mathbb{N}$ and $\beta \in \mathbb{R}$ we set

$$
\stackrel{\mathscr{H}}{ }_{k}^{\beta}:=\stackrel{\circ}{H}_{r, r^{-n / 2-\beta}}^{k},
$$

so that the $\mathscr{\mathscr { H }}_{k}^{\beta}$ spaces are the weighted Sobolev spaces of (A.1) with $\phi=r \circ \chi^{-1}$ and $\psi$ a power of $r \circ \chi^{-1}$; the labeling here follows [7], and is motivated by the simple inclusions $[7]$

$$
C_{k}^{\beta^{\prime}} \subset \dot{\mathscr{H}}_{k}^{\beta}, \beta^{\prime}<\beta ; \quad \dot{\mathscr{H}}_{k}^{\beta} \subset C_{\lfloor k-n / 2\rfloor}^{\beta}, k>n / 2 .
$$

In fact $[7]$

$$
f \in \dot{\mathscr{H}}_{k}^{\beta}, k>n / 2 \quad \Longrightarrow \quad f=o\left(r^{\beta}\right) .
$$

In order to apply Theorem 3.6 we need to establish the inequality (3.4) for tensor fields with compact support in the asymptotic region, and we will use Lemma 4.3 for that. In addition to (C.7), we assume that the Hessian Hess $r$ satisfies, in the preferred coordinates on the asymptotic region,

$$
(\operatorname{Hess} r)_{i j}=\frac{1}{r}\left(\delta_{i}^{j}-n_{i} n_{j}\right)+o\left(r^{-1}\right) .
$$

(Equation (7.5) will clearly hold without the error term for a flat metric; similarly (7.5) will hold for metrics which are $C_{1}^{\alpha}$-asymptotically flat, for some $\alpha>0$; we note that (7.5) implies (C.8).) For the convenience of the reader we restate Proposition D.9:

Proposition 7.2. - Let $S$ be defined by Equation (4.4). Assume that $g \in W_{\text {loc }}^{1, \infty}$, that

$$
\left|g_{i j}-\delta_{j}^{i}\right| \leqslant \varepsilon \text { on }\left\{r \geqslant R_{\varepsilon}\right\}
$$

and that (7.5) holds. Then for $s \in \mathbb{R} \backslash\{0,1\}$ there exist constants $R=R(s)$ and $C_{s}$ such that for all differentiable vector fields $Y$ compactly supported outside of a ball of radius $R$ we have

$$
\int r^{-2 s-n}|Y|^{2} \leqslant C_{s} \int r^{-2 s-n+2}|S(Y)|^{2}
$$

REMARK 7.3. - The result is sharp, compare the argument in Remark 5.2.

Proposition 7.2 gives, in essence, the inequality needed in Theorem 3.6 for $s \notin$ $\{0,1\}$. This leads to the following rewording of Theorem 3.6 in the asymptotically 
flat context (there is also an equivalent of Theorem 3.4 here, we leave the transcription to the reader):

THEOREM 7.4. - Let $g_{0}$ be $r^{-\alpha} W_{r}^{k+4, \infty}$-asymptotically flat for some $\alpha>0$ and $k \in \mathbb{N}$, suppose that

$$
K_{0} \in r^{-1} W_{r}^{k+3, \infty}, \quad K_{0}=o\left(r^{-1}\right) .
$$

Then for all $\sigma \in \mathbb{R} \backslash\{0,1\}$ and for all $(K, g)$ close to $\left(K_{0}, g_{0}\right)$ in $r^{-1} W_{r}^{k+3, \infty}\left(g_{0}\right) \times$ $W_{r}^{k+4, \infty}\left(g_{0}\right)$ norm, the map

$$
\pi_{\mathcal{K}_{0}^{\perp_{g}}} L_{r, r^{-n / 2-\sigma}}: \mathcal{K}_{0}^{\perp_{g}} \cap\left(\dot{\mathscr{H}}_{k+3}^{\sigma}(g) \times \dot{\mathscr{H}}_{k+4}^{\sigma}(g)\right) \longrightarrow \mathcal{K}_{0}^{\perp_{g}} \cap\left(\dot{\mathscr{H}}_{k+1}^{\sigma}(g) \times \dot{\mathscr{H}}_{k}^{\sigma}(g)\right)
$$

is an isomorphism such that the norm of its inverse does not depend upon $(K, g)$.

Before passing to its proof, we note that Theorem 7.4 immediately implies:

Corollary 7.5. - Under the hypotheses of Theorem 7.4, the space of linearized fields $(\delta K, \delta g) \in \dot{\mathscr{H}}_{k+1}^{-n-\sigma+1} \times \dot{\mathscr{H}}_{k+2}^{-n-\sigma+2}$ splits as a direct sum $\operatorname{KerP} \oplus B$, with the restriction of the linearization $P$ of the constraint map to $B$ being an isomorphism of $B$ and of

$$
\mathcal{K}^{\perp_{g}} \cap\left(\dot{\mathscr{H}}_{k+1}^{-n-\sigma} \times \dot{\mathscr{H}}_{k}^{-n-\sigma}\right) .
$$

In particular if there are no solutions $(Y, N) \in \dot{\mathscr{H}}_{1}^{\sigma} \times \dot{\mathscr{H}}_{2}^{\sigma}$ of the equation $P^{*}(Y, N)=$ 0 , then the map

$$
\dot{\mathscr{H}}_{k+2}^{-n-\sigma+1} \times \dot{\mathscr{H}}_{k+2}^{-n-\sigma+2} \ni(\delta K, \delta g) \longrightarrow(\delta J, \delta \rho):=P(\delta K, \delta g) \in \dot{\mathscr{H}}_{k+1}^{-n-\sigma} \times \dot{\mathscr{H}}_{k}^{-n-\sigma}
$$

is surjective.

Proof of Theorem 7.4. - We wish to apply Theorem 3.6, in order to do that we need to establish the inequality (3.4) for $Y$ 's and $N$ 's supported outside of a sufficiently a large ball. For $\sigma \neq 0,1$, Proposition 7.2 yields

$$
\|Y\|_{\dot{\mathscr{H}}_{0}^{\sigma}} \leqslant C\|S(Y)\|_{\dot{\mathscr{H}}_{0}^{\sigma-1}} .
$$

Applying Proposition C.5 twice we find that for $\sigma \neq 0,1$ it holds that

$$
\|N\|_{\dot{\mathscr{C}}_{1}^{\sigma}} \leqslant C\|\nabla N\|_{\mathscr{\mathscr { H }}_{0}^{\sigma-1}} \leqslant C^{2}\|\nabla \nabla N\|_{\dot{\mathscr{C}}_{0}^{\sigma-2}} \leqslant C^{3}\|\alpha(\nabla \nabla N)\|_{\dot{\mathscr{H}}_{0}^{\sigma-2},},
$$

where $\alpha$ is as in (2.15). Now $(M, g, K, r)$ satisfy the asymptotic condition a) of Definition 4.1 with $U_{i}=\left(\chi^{-1}\right)^{-1}\left(B(i) \backslash \overline{B\left(R_{0}\right)}\right) \cup U$, where $U$ is a relatively compact open neighborhood of $\mathscr{K}\left(\chi, R_{0}\right.$ and $\mathscr{K}$ as in Definition 7.1), and Lemma 4.3 shows that we can apply Theorem 3.6.

Elements in the kernel of $P^{*}$ are called Killing initial data (KIDs) [9]. Existence of non-zero KIDs implies existence of non-zero Killing vectors in the associated vacuum space-time (see $[\mathbf{8}, \mathbf{9}]$ and references therein). We have the following corollary of Theorem 7.4: 
Corollary 7.6. - Let $g_{0}$ be $r^{-\alpha} W_{r}^{k+4, \infty}$-asymptotically flat for some $\alpha>0$ and $k \in \mathbb{N}$ and suppose that Equation (7.8) holds.

(1) If $\sigma \leqslant 0$, or if

(2) $\sigma \notin\{0,1\}$ and the set of nontrivial KIDs is empty,

then the conclusions of Theorem 7.4 hold without any projection.

Proof. - There are no nontrivial KIDs in $\dot{\mathscr{H}}_{1}^{\sigma} \times \check{\mathscr{H}}_{2}^{\sigma}$ for $\sigma \leqslant 0$, then $\mathcal{K}_{0}=\{0\}$.

Theorem 7.7. - Let $g_{0}$ be $r^{-\alpha} W_{r}^{k+4, \infty}$-asymptotically flat for some $\alpha>0$ and $k>n / 2$, suppose that

$$
K_{0} \in r^{-1} W_{r}^{k+3, \infty}, \quad K_{0}=o\left(r^{-1}\right) .
$$

Then for all $\sigma \geqslant 2-n, \sigma \notin\{0,1\}$ the nonlinear map

$$
\begin{gathered}
\mathcal{K}_{0}^{\perp_{g}} \cap\left(\dot{\mathscr{H}}_{k+3}^{\sigma}(g) \times \dot{\mathscr{H}}_{k+4}^{\sigma}(g)\right) \longrightarrow \mathcal{K}_{0}^{\perp_{g}} \cap\left(\dot{\mathscr{H}}_{k+1}^{\sigma}(g) \times \dot{\mathscr{H}}_{k}^{\sigma}(g)\right) \\
(Y, N) \longmapsto \pi_{\mathcal{K}_{0}^{\perp g}} r^{n+2 \sigma}\left\{\left(\begin{array}{c}
J \\
\rho
\end{array}\right)\left[(K, g)+r^{-n-2 \sigma} \Phi^{2} P^{*}(Y, N)\right]-\left(\begin{array}{c}
J \\
\rho
\end{array}\right)(K, g)\right\}
\end{gathered}
$$

is bijective in a neighborhood of zero. Thus, there exists $\varepsilon>0$ such that for all $(K, g)$ close to $\left(K_{0}, g_{0}\right)$ in $r^{-1} W_{r}^{k+3, \infty}\left(g_{0}\right) \times W_{r}^{k+4, \infty}\left(g_{0}\right)$, and for all pairs

$$
(\delta J, \delta \rho) \in \dot{\mathscr{H}}_{k+1}^{-n-\sigma}(g) \times \dot{\mathscr{H}}_{k}^{-n-\sigma}(g)
$$

with norm less than $\varepsilon$, there exists a solution

$$
\left.(\delta K, \delta g)=r^{-n-2 \sigma} \Phi^{2} P^{*}(Y, N) \in \dot{\mathscr{H}}_{k+2}^{-n-\sigma+1}(g) \times \dot{\mathscr{H}}_{k+2}^{-n-\sigma+2}(g)\right),
$$

close to zero, of the equation

$$
\pi_{\mathcal{K}_{0}^{\perp g}} r^{n+2 \sigma}\left\{\left(\begin{array}{c}
J \\
\rho
\end{array}\right)(K+\delta K, g+\delta g)-\left(\begin{array}{c}
J \\
\rho
\end{array}\right)(K, g)\right\}=\pi_{\mathcal{K}_{0}^{\perp g}} r^{n+2 \sigma}\left(\begin{array}{c}
\delta J \\
\delta \rho
\end{array}\right)
$$

Proof. - The conditions $\sigma \geqslant 2-n$ and $k>n / 2$ ensure that the map of Equation (7.12) is well defined and differentiable in a neighborhood of zero. The result follows then from Theorem 3.9.

Clearly, the projection operator in (7.13) is not needed when the hypotheses of Corollary 7.6 are satisfied.

We also have solutions with Hölder regularity:

Proposition 7.8. - Let $g_{0}$ be $C_{k+4, \beta}^{-\alpha}$-asymptotically flat for some $\alpha>0, \beta \in(0,1)$, and $k \geqslant k_{0}:=\lfloor n / 2\rfloor+1$ (the smallest integer strictly larger than $n / 2$ ), suppose that

$$
K_{0} \in C_{k+3, \beta}^{-1}, \quad K_{0}=o\left(r^{-1}\right) .
$$


Then for all $t \geqslant 2, t \notin\{n, n+1\}$ there exists $\varepsilon>0$ such that if $(K, g)$ in $C_{k+3, \beta}^{-1}\left(g_{0}\right) \times$ $C_{k+4, \beta}^{0}\left(g_{0}\right)$, and if

$$
\begin{array}{r}
\left\|\left(K-K_{0}, g-g_{0}\right)\right\|_{r^{-1} W_{r}^{k_{0}+3, \infty}\left(g_{0}\right) \times W_{r}^{k_{0}+4, \infty}\left(g_{0}\right)}<\varepsilon \\
\|(\delta J, \delta \rho)\|_{C_{k_{0}+1, \beta}^{-t}(g) \times C_{k_{0}, \beta}^{-t}(g)}+\|(\delta J, \delta \rho)\|_{\mathscr{\mathscr { H }}_{k_{0}+1}^{-t}(g) \times \mathscr{\mathscr { H }}_{k_{0}}^{-t}(g)}<\varepsilon
\end{array}
$$

then the solution $(\delta K, \delta g)$ given by Theorem 7.7 (with $\sigma=t-n)$ is in $C_{k_{0}+2, \beta}^{-t+1}(g) \times$ $C_{k_{0}+2, \beta}^{-t+2}(g)$. If moreover $(\delta J, \delta \rho) \in C_{k+1, \beta}^{-t}(g) \times C_{k, \beta}^{-t}(g)$ then the solution given by Theorem 7.7 is in $C_{k+2, \beta}^{-t+1}(g) \times C_{k+2, \beta}^{-t+2}(g)$.

Proof. - Under the current hypotheses all the conditions of Proposition 3.16 with $k$ there equal to $k_{0}$ are met. (The weighted elliptic regularity condition of Definition 3.12 is satisfied by the calculation (B.4), Appendix B.) The higher Hölder regularity follows from Proposition 3.17.

REMARK 7.9. - One has very similar results in exponentially weighted Hölder and Sobolev spaces as at the end of Section 5, the details are left to the reader. However, the exponentially weighted conditions seem difficult to verify in the current case, unless one is in a setting where the results of Section 5 can be applied. In such a case sharper results are obtained by using the theorems of that section. 



\section{CHAPTER 8}

\section{APPLICATIONS}

In this section we will give several applications of the general results proved so far. It should be clear that a key role in this approach is played by the kernel of $P^{*}$. As already mentioned in the previous section, elements of this kernel will be called Killing Initial Data (KIDs). Thus, a KID is a pair $(Y, N)$ such that

$$
P^{*}(Y, N)=0
$$

Our first application of the techniques developed so far concerns the construction of initial data which are exactly Kerrian outside of a compact set:

\subsection{Space-times that are Kerrian near $i^{0}$}

(A version of) the following result has been announced in [31]; we assume that the initial data manifold is three-dimensional:

THeOREM 8.1. - Let $g$ be $r^{-\alpha} W_{r}^{k+4, \infty}$-asymptotically flat for some $\alpha>1 / 2$ and $2 \leqslant k \in \mathbb{N}$, with $K \in r^{-\alpha-1} W_{r}^{k+3, \infty}$, and suppose that $(K, g)$ satisfies the vacuum constraint equations. We further assume that $(K, g)$ satisfy the $3+1$ equivalent of the parity conditions (E.9),

$$
\left|g_{i j}^{-}\right|+r\left|\partial_{k}\left(g_{i j}^{-}\right)\right|+r\left|K_{i j}^{-}\right| \leqslant C(1+r)^{-\alpha_{-}}, \quad \alpha_{-}>\alpha, \alpha+\alpha_{-}>2,
$$

so that all Poincaré charges of $(K, g)$ are finite and well defined, with the ADM fourmomentum being timelike. Then there exists $R_{1}<\infty$ such that for all $R \geqslant R_{1}$ there exists an initial data set

$$
\left(\widehat{K}_{R}, \widehat{g}_{R}\right) \in C^{k+2} \times C^{k+2}
$$

satisfying the vacuum constraint equations everywhere such that $\left(\widehat{K}_{R}, \widehat{g}_{R}\right)$ coincides with $(K, g)$ for $r \leqslant R$, and $\left(\widehat{K}_{R}, \widehat{g}_{R}\right)$ coincides with initial data for some Kerr metric for $r \geqslant 4 R$. If $K$ and $g$ are smooth, then $\left(\widehat{K}_{R}, \widehat{g}_{R}\right)$ is smooth. 
REMARK 8.2. - A family of $(n+1)$-dimensional generalizations of the Kerr metric has been found by Myers and Perry [55], we expect that it can be used to prove a corresponding result in $(n+1)$ dimensions. The argument below carries over to any dimension, the only element missing is an equivalent of Proposition F.1 for the family of translated, rotated, and boosted Myers-Perry metrics.

REMARK 8.3. - The factor 4 has been chosen for definiteness; an identical result holds with $\left(\widehat{K}_{R}, \widehat{g}_{R}\right)$ being Kerr for $r \geqslant \lambda R$ for any constant $\lambda>1$.

REMARK 8.4. - The ADM momentum and angular momentum of $\left(\widehat{K}_{R}, \widehat{g}_{R}\right)$ converges to that of $(K, g)$ as $R$ tends to infinity.

Proof. - The required initial data will be constructed by a gluing procedure, on an annulus, using a method due to Corvino $[\mathbf{3 0}, \mathbf{3 1}]$, together with Theorem 3.19. Let $e_{(i)}, i=1, \ldots, 10$, be any basis of the space of KIDs for Minkowski space-time, let $Q_{(i)}$ denote the Hamiltonian charge $Q\left(Y_{(i)}, N_{(i)}, K, g\right)$ of $(K, g)$ as given by (E.25) with $\left(Y_{(i)}, N_{(i)}\right)=e_{(i)}$. Let, as in Appendix F, $\mathscr{K}_{i^{0}}$ denote the set of initial data for boosted, rotated, and translated Kerr metrics, and let $\left(K_{Q}, g_{Q}\right)$ denote an initial data set in $\mathscr{K}_{i^{0}}$ with Hamiltonian charge $Q=\left(Q_{(i)}\right) \in \mathbb{R}^{10}$. For $R \in\left[R_{0}, \infty\right)$ let the scale-down map $\Phi_{R}$ be defined as

$$
\begin{aligned}
\Phi_{R}: \Gamma(R, 4 R):=\overline{B(0,4 R) \backslash B(0, R)} & \longrightarrow \Gamma(1,4), \\
x & \longmapsto x / R .
\end{aligned}
$$

Let $\chi \in C^{\infty}\left(\mathbb{R}^{3}\right)$ be a spherically symmetric cut-off function such that $0 \leqslant \chi \leqslant 1$, $\chi \equiv 1$ on $\Gamma(1,2)$, and $\chi \equiv 0$ on $\Gamma(3,4)$. On $\Gamma(1,4)$ set

$$
\begin{aligned}
g_{Q, R} & =R^{-2}\left(\chi \Phi_{R}^{*} g+(1-\chi) \Phi_{R}^{*} g_{Q}\right), \\
K_{Q, R} & =R^{-1}\left(\chi \Phi_{R}^{*} K+(1-\chi) \Phi_{R}^{*} K_{Q}\right) .
\end{aligned}
$$

Then the $g_{Q, R}$ 's form a family of metrics that converge, as $R$ tends to infinity, in weighted Sobolev topologies with arbitrary decay (at the boundary) index $t>1$, to the Euclidean metric $g_{0}$ on $\Gamma(1,4)$, while the $K_{Q, R}$ 's converge to $K_{0} \equiv 0$ :

$$
\left\|g_{Q, R}-g_{0}\right\|_{\dot{\mathrm{H}}_{k+2}^{t}}+\left\|K_{Q, R}\right\|_{\dot{\mathrm{H}}_{k+2}^{t-1}} \leqslant C_{s, k, Q} R^{-\beta}, \quad \beta:=\min (\alpha, 1)
$$

(recall that the weighted Sobolev spaces used here have been defined in (5.2)). Further the convergence is uniform in $Q$ on any compact set of $Q$ 's. We shall write $x$ for $(K, g)$, $x_{Q}$ for $\left(K_{Q}, g_{Q}\right)$, etc. We choose the index $t$ to be larger than or equal to $k+5-$ say $t=k+6$, in particular initial data in the space $\stackrel{\circ}{\mathrm{H}}_{k+2}^{t-1} \times \stackrel{\circ}{\mathrm{H}}_{k+2}^{t}$ vanish on $\partial \Gamma(1,4)$, as well as their first derivatives. Further, this ensures the continuous embedding $\stackrel{\circ}{\mathrm{H}}_{k+2}^{t-1} \subset H^{k+2}$.

It follows from (8.3) that for $R$ sufficiently large we have

$$
\left\|J\left(x_{Q, R}\right)\right\|_{\stackrel{\mathrm{H}}{k+1}_{k-2}^{t-2}}+\left\|\rho\left(x_{Q, R}\right)\right\|_{\dot{\mathrm{H}}_{k}^{t-2}} \leqslant C R^{-\beta},
$$


and Theorem 5.6 with $s=t-1$ provides a solution $\delta x_{Q, R} \in \stackrel{\circ}{H+2}_{k+1} \times \stackrel{\circ}{H}_{k+2}^{t}$ of Equation (5.8) satisfying

$$
\left\|\delta x_{Q, R}\right\|_{\stackrel{\mathrm{H}}{k+2}_{k+2}^{t-1} \times \stackrel{\mathrm{H}}{k+2}^{t}} \leqslant C R^{-\beta} .
$$

Set $\delta J=J\left(x_{Q, R}+\delta x_{Q, R}\right), \delta \rho=\rho\left(x_{Q, R}+\delta x_{Q, R}\right)$, let the parameter $\lambda$ in Theorem 3.19 be equal to $R$, and consider the map $F_{\lambda}$ defined in (3.29). We have

$$
\begin{aligned}
\left\langle e_{(i)}, \psi^{-2}\left(\begin{array}{c}
\delta J \\
\delta \rho
\end{array}\right)\right\rangle_{L_{\psi}^{2} \oplus L_{\psi}^{2}} & =\left\langle\left(Y_{(i)}, N_{(i)}\right), \psi^{-2}\left(\begin{array}{c}
\delta J \\
\delta \rho
\end{array}\right)\right\rangle_{L_{\psi}^{2} \oplus L_{\psi}^{2}} \\
& =\int_{\Gamma(1,4)}\left(Y_{(i)}^{j} \delta J_{j}+N_{(i)} \delta \rho\right) d \mu_{g} .
\end{aligned}
$$

Note that the weight factor $\psi$ has vanished from the last integral. By (E.26) we have

$$
\begin{aligned}
\left\langle e_{(i)}, \psi^{-2}\left(\begin{array}{c}
\delta J \\
\delta \rho
\end{array}\right)\right\rangle_{L_{\psi}^{2} \oplus L_{\psi}^{2}}= & \int_{\{r=4\}} \mathbb{U}^{\alpha \beta}\left(x_{Q, R}+\delta x_{Q, R}\right) d S_{\alpha \beta} \\
& -\int_{\{r=1\}} \mathbb{U}^{\alpha \beta}\left(x_{Q, R}+\delta x_{Q, R}\right) d S_{\alpha \beta}+O\left(R^{-2 \beta}\right),
\end{aligned}
$$

with the error term $O\left(R^{-2 \beta}\right)$ being uniform in $Q$ whenever $Q$ ranges over a compact set. Now, on $S(0,1)$ all the initial data considered coincide - up to a rescaling with $(g, K)$ together with their first derivatives, so that by (E.19) we have

$$
\int_{\{r=1\}} \mathbb{U}^{\alpha \beta} d S_{\alpha \beta}=R^{-1}\left\{Q_{(i)}^{0}+O\left(R^{-\delta}\right)\right\},
$$

where $Q_{(i)}^{0}$ denotes the Hamiltonian charge of $(K, g)$, for some $\delta>0$. Similarly, on $S(0,4)$ all the initial data considered coincide (up to scale-down) with $\left(K_{Q}, g_{Q}\right)$ together with their first derivatives, so that

$$
\int_{\{r=4\}} \mathbb{U}^{\alpha \beta} d S_{\alpha \beta}=R^{-1}\left\{Q_{(i)}+O\left((4 R)^{-\delta}\right)\right\},
$$

It follows that

$$
\left\langle e_{(i)}, \psi^{-2}\left(\begin{array}{c}
\delta J \\
\delta \rho
\end{array}\right)\right\rangle_{L_{\psi}^{2} \oplus L_{\psi}^{2}}=R^{-1}\left\{Q_{(i)}-Q_{(i)}^{0}+O\left(R^{-\min (\delta, 2 \beta-1)}\right)\right\} .
$$

This implies that, up to an additive constant, the maps $\lambda F_{\lambda}$ converge as $\lambda=R$ tends to infinity to the map $Q$ of Proposition F.1; that last map is a homeomorphism, as desired. The conclusion is obtained now from Theorem 3.19 by taking $V \subset \mathbb{R}^{10}$ to be a ball around $\left(Q_{(i)}^{0}\right)$ of a radius small enough so that $V$ is included in the image of the map $Q$ of Proposition F.1, with $U=Q^{-1}(V)$.

Finally, if $g$ and $K$ are smooth, then smooth solutions can be obtained by using the exponentially weighted spaces of Proposition 5.10, compare Corollary 5.11. In the construction above one should choose the cut-off function $\chi$ to be constant in a neighborhood of the boundary of the annulus $\Gamma(1,4)$. 


\subsection{Gluing asymptotically flat initial data sets}

The gluing technique used in Section 8.1 does apply to much more general situations, as follows: a family of vacuum initial data $\left\{\left(K_{\omega}, g_{\omega}\right)\right\}_{\omega \in \Omega}$ will be called a reference family if the following holds:

(1) There exists $R>0$ such that all the data sets $\left(K_{\omega}, g_{\omega}\right)$ are defined on $\mathbb{R}^{3} \backslash$ $B(0, R)$.

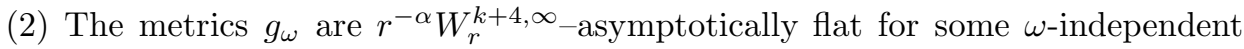
constant $\alpha>1 / 2$ and $2 \leqslant k \in \mathbb{N}$, with $K_{\omega} \in r^{-\alpha-1} W_{r}^{k+3, \infty}$, and with the norms in those spaces being bounded independently of $\omega \in \Omega$.

(3) The parity conditions (8.1) hold with $g$ and $K$ there replaced by $g_{\omega}$ and $K_{\omega}$, for some $\omega$-independent constants $\alpha_{-}$and $C$.

(4) The map which to $\left(K_{\omega}, g_{\omega}\right)$ assigns its Poincaré charges $\left(p^{\mu}(\omega), J^{\mu \nu}(\omega)\right)$ is a diffeomorphism between $\Omega$ and an open subset of $\mathbb{R}^{10}$ :

$$
\mathscr{U}_{\Omega}:=\left\{\left(p^{\mu}(\omega), J^{\mu \nu}(\omega)\right)\right\}_{\omega \in \Omega} \subset \mathbb{R}^{4} \times \mathbb{R}^{6} .
$$

There is an obvious equivalent of the definition above in the time symmetric context: in this case one assumes that $K_{\omega} \equiv 0$, and one requires $\mathscr{U}_{\Omega}$ to be an open subset of $\mathbb{R}^{4}$, with one parameter corresponding to mass, and three parameters corresponding to the centre of mass.

It is proved in Appendix $\mathrm{E}$ that the collection of initial data obtained from boosted and space-translated Kerr space-times provides an example of a reference family. More generally, consider any one parameter family of vacuum initial data sets $\left(K_{\lambda}, g_{\lambda}\right)$ on $\mathbb{R}^{3} \backslash B(0, R), \lambda \in(-\varepsilon, \varepsilon)$, which satisfies the decay and parity conditions of the definition of a reference family. For definiteness we shall suppose that the ADM four-momentum $p^{\mu}(\lambda)$ of $\left(K_{\lambda}, g_{\lambda}\right)$ is a $\lambda$-independent timelike future pointing vector, and that the length squared $J^{i j}(\lambda) J_{i j}(\lambda)$ of the ADM angular momentum $J^{i j}(\lambda)$ of $\left(K_{\lambda}, g_{\lambda}\right)$ varies smoothly in some open interval as $\lambda$ changes. Then scaling $\left(x^{i} \rightarrow a x^{i}\right.$, $g \rightarrow a^{-2} g$ for $a \in \mathbb{R}^{+}$), translating, and rotating the initial data, and boosting the initial data hypersurface in the associated maximal globally hyperbolic development, leads to a reference family such that the associated Poincaré charges form a neighborhood of $\left(p^{\mu}(0), J^{\mu \nu}(0)\right)$; this follows from the boost theorem $[\mathbf{1 5}, \mathbf{1 7}]$ together with the analysis in Appendix E (here one needs to apply the boost theorem first to the full metric, and then to its odd part).

Similarly, in the time symmetric context, an example of a reference family is provided by translated Schwarzschild initial data. More generally, if $g$ is a scalar flat metric on $\mathbb{R}^{3} \backslash B(0, R)$ which satisfies the decay and parity conditions of the definition of a reference family and which has non-vanishing ADM mass, then scaling and translating provides a time-symmetric reference family.

A repetition of the proof of Theorem 8.1 gives:

MÉMOIRES DE LA SMF 94 
THEOREM 8.5. - Under the hypotheses of Theorem 8.1, consider any reference family $\left\{\left(K_{\omega}, g_{\omega}\right)\right\}_{\omega \in \Omega}$ such that the associated set $\mathscr{U}_{\Omega}$ defined by (8.8) forms a neighborhood of the Poincaré charge $\left(p^{\mu}, J^{\mu \nu}\right)$ of $(K, g)$. Then the conclusion of Theorem 8.1 holds with the new initial data set coinciding in the asymptotic region with one of the members of the reference family rather than with one of the members of the Kerr family.

The point of Theorem 8.5 is that it provides large families of initial data with well controlled asymptotic behavior. As an example of application, let the reference family consist of stationary metrics. Such metrics have well understood asymptotic behavior (cf., e.g., $[\mathbf{6 0}]$ ), and large families of non-trivial solutions (defined and smooth outside of a compact set) have been constructed in $[\mathbf{4 9 , 5 7 , 5 9 ]}$. Theorem 8.5 allows one to modify an arbitrary initial data set in the asymptotic region so that it coincides with exactly stationary, but not necessarily Kerrian, data there. Further, there is a rather large freedom available. Now, a significant result of Dain, Damour and Schmidt $[\mathbf{3 4}, \mathbf{3 5}]$ implies that the resulting vacuum space-time will have a smooth $\mathscr{I}^{+}$complete to the past. Thus, initial data so constructed do have reasonably well controlled maximal globally hyperbolic developments. We will see in Section 8.6 below how to construct initial data that produce space-times with a complete smooth $\mathscr{I}$, by using a variation of the technique above.

Another possibility is to choose as the reference family appropriate subsets of the set of "almost stationary" metrics constructed in Section 8.3 below, see Theorem 8.9. The metrics there are stationary (or static, in the time-symmetric case) to an order as high as desired in an asymptotic expansion, without being exactly stationary outside of a compact set, which further increases the freedom available. One expects (compare [40]) that some of those metrics will also admit complete, or past-complete, conformal completions with a reasonably high degree of differentiability, but no rigorous statements of this kind are known so far.

All the constructions described so far can be repeated by specializing to the timesymmetric case, setting

$$
Y \equiv K \equiv 0
$$

throughout. In this context Theorem 8.5 can be rephrased as:

THEOREM 8.6. - Let $g$ be $r^{-\alpha} W_{r}^{k+4, \infty}$-asymptotically flat for some $\alpha>1 / 2$ and $2 \leqslant k \in \mathbb{N}$, and suppose that

$$
R(g)=0 .
$$

We further assume that $g$ satisfies the parity conditions

$$
\left|g_{i j}^{-}\right|+r\left|\partial_{k}\left(g_{i j}^{-}\right)\right| \leqslant C(1+r)^{-\alpha_{-}}, \quad \alpha_{-}>\alpha, \alpha+\alpha_{-}>2,
$$

so that the mass $m$ and the centre of mass $\vec{c}$ of $g$ are finite and well defined, with $m \neq 0$. Consider any time-symmetric reference family $\left\{\left(K_{\omega} \equiv 0, g_{\omega}\right)\right\}_{\omega \in \Omega}$ such that 
the associated set $\mathscr{U}_{\Omega}$ of masses and centres of mass forms a neighborhood of $(m, \vec{c})$. Then there exists $R_{1}<\infty$ such that for all $R \geqslant R_{1}$ there exists a scalar flat metric

$$
\widehat{g}_{R} \in C^{k+2}
$$

such that $\widehat{g}_{R}$ coincides with $g$ for $r \leqslant R$, and $\widehat{g}_{R}$ coincides with a member of the reference family for $r \geqslant 4 R$. If $g$ is smooth, and the $g_{\omega}$ 's are smooth, then $\widehat{g}_{R}$ can be chosen to be smooth.

\subsection{Initial data which are stationary to high asymptotic order}

The results in Section 7 can be used to construct large classes of asymptotically flat vacuum initial data sets with controlled asymptotic behavior. As an illustration, let $(K, g)$ be a solution of the stationary constraint equations defined on $\mathbb{R}^{3} \backslash B(0, R)$ for some $R$. Recall that such solutions are uniquely determined [12] by an infinite collection of Hansen multipole moments $\left\{\mathscr{P}_{m}(K, g)\right\}_{m \in \mathbb{N}}$, as defined in [60, Equation (3.5)], see also [43]. We will assume that the reader is familiar with $[\mathbf{6 0}]$ and we will use notation from there. In that reference it has been shown how to construct approximate solutions of the stationary equations to any asymptotic order: given any set $\mathscr{P}_{m}$ one can find functions $\Phi_{M}^{(m)}, \Phi_{S}^{(m)}, \Phi_{K}^{(m)}, \gamma^{(m)}$ which satisfy the reduced Einstein equations [60, Equations (3.1)-(3.4)] to order $O\left(r^{-(m+3)}\right)$. We wish, first, to show that this implies existence of initial data $\left(K\left(\mathscr{P}_{m}\right), g\left(\mathscr{P}_{m}\right)\right)$ satisfying the stationary - or static — Einstein equations up to terms $O\left(r^{-m-3}\right)$, provided that the NUT charge vanishes; in particular this will imply, in the notation of [60],

$$
\left(\begin{array}{c}
J \\
\rho
\end{array}\right)\left(K\left(\mathscr{P}_{m}\right), g\left(\mathscr{P}_{m}\right)\right)=\left(\begin{array}{c}
O^{\infty}\left(r^{-m-3}\right) \\
O^{\infty}\left(r^{-m-3}\right)
\end{array}\right) \in \dot{\mathscr{H}}_{k}^{-(m-\varepsilon)-3}, \varepsilon>0, k \in \mathbb{N} .
$$

We will say that the moments are static to order $m$ if the associated twist function $\omega^{(m)}$ vanishes. In this last case the proof of $(8.10)$ is straightforward: we set $K\left(\mathscr{P}_{m}\right):=0$, and (8.10) follows immediately from the equations in [60] with $\sigma=\omega=0$ there. In the general case some more work is required, we start with a lemma:

Lemma 8.7 (Approximate Poincaré Lemma). — Let

$$
\nu=A d y \wedge d z+B d z \wedge d x+C d x \wedge d y
$$

be a two form on $\mathbb{R}^{3}$, with coefficients of order $o\left(r^{-2}\right)$, such that

$$
d \nu=\left(\partial_{x} A+\partial_{y} B+\partial_{z} C\right) d x \wedge d y \wedge d z=O\left(r^{-(m+3)}\right) d x \wedge d y \wedge d z
$$

with $m>0$. Then there exists a one form $\sigma$ such that

$$
d \sigma=\nu+O\left(r^{-(m+2)}\right) .
$$

MÉMOIRES DE LA SMF 94 
Proof. - In the proof that follows we use the notation $X=(x, y, z)$ and $r=$ $\sqrt{x^{2}+y^{2}+z^{2}}$. Let us show that the one form $\sigma$ defined by the usual formula

$$
\begin{array}{r}
\sigma=-\left\{\left[\int_{1}^{\infty} A(t X) t d t\right](y d z-z d y)+\left[\int_{1}^{\infty} B(t X) t d t\right](z d x-x d z)\right. \\
\left.+\left[\int_{1}^{\infty} C(t X) t d t\right](x d y-y d x)\right\}
\end{array}
$$

satisfies the desired estimate. Indeed, the coefficient of the term $d x \wedge d y$ in $d \sigma$ is

$$
\begin{gathered}
-\int_{1}^{\infty}\left\{t^{2}\left[x \partial_{x} C(t X)-z \partial_{x} A(t X)-z \partial_{y} B(t X)+y \partial_{y} C(t X)\right]+2 t C(t X)\right\} d t \\
=-\int_{1}^{\infty}\left\{t^{2}\left[x \partial_{x} C(t X)+y \partial_{y} C(t X)+z \partial_{z} C(t X)+z O\left((t r)^{-(m+3)}\right)\right]+2 t C(t X)\right\} d t \\
=-\left.t^{2} C(t X)\right|_{1} ^{\infty}-\int_{1}^{\infty} z t^{2} O\left((t r)^{-(m+3)}\right) d t=C(X)+O\left(r^{-(m+2)}\right) .
\end{gathered}
$$

A similar calculation for the remaining terms gives the result.

REMARK 8.8. - If $\widetilde{\nu}$ is another two form satisfying conditions of Lemma 8.7 such that $\widetilde{\nu}-\nu=O\left(r^{-(m+2)}\right)$, then if we define $\widetilde{\sigma}$ as in the preceding proof we will have

$$
\tilde{\sigma}-\sigma=O\left(r^{-(m+1)}\right) \text {. }
$$

This shows in particular that in a stationary vacuum metric the approximate solution $\sigma^{(m)}$ as defined below will differ from the exact one by $O\left(r^{-(m+1)}\right)$.

Returning to our construction, let $\Phi_{M}^{(m)}, \Phi_{S}^{(m)}, \Phi_{K}^{(m)}$, and $\gamma^{(m)}$ be as in [60, Theorem 2], then Beig \& Simon's equations (2.8) and (2.9) are satisfied modulo $O\left(r^{-(m+3)}\right)$. In particular their equation (2.8) with $\Phi=\Phi_{K}^{(m)}-\Phi_{M}^{(m)}$ gives

$$
\Delta_{\gamma^{(m)}}\left(\lambda^{-1}\right)=2 \tau^{(m)} \lambda^{-1}+O\left(r^{-(m+3)}\right),
$$

where $\lambda \equiv \lambda^{(m)}$ is obtained from $\Phi_{M}^{(m)}, \Phi_{S}^{(m)}$, and $\Phi_{K}^{(m)}$ by inverting [60, Equation (2.6)] with $\Phi_{M}$ there replaced with $\Phi_{M}^{(m)}$, etc.; we define $\omega \equiv \omega^{(m)}$ in a similar way. Using again Beig and Simon's Equation (2.8) with $\Phi=\Phi_{S}^{(m)}$ gives

$$
\Delta_{\gamma^{(m)}}\left(\lambda^{-1} \omega\right)=2 \tau^{(m)} \lambda^{-1} \omega+O\left(r^{-(m+3)}\right) .
$$

Developing (8.12), and inserting (8.11) in the result one obtains

$$
\nabla^{i}\left(\lambda^{-2} \partial_{i} \omega\right)=O\left(r^{-(m+3)}\right) .
$$

Here $\nabla \equiv \nabla\left(\gamma^{(m)}\right)$ is the connection of the metric $\gamma^{(m)}$. Then, if we define $\nu \equiv$ $\nu^{(m)}:=-\lambda^{-2} *_{\gamma^{(m)}} d \omega$, we have

$$
d \nu=O\left(r^{-(m+3)}\right)
$$

Recall, now, that the coefficient of the power $r^{-1}$ in the expansion of $\omega$ is proportional to the NUT charge of the resulting space-time; usual asymptotic flatness forces the vanishing thereof. From now on we assume that this is the case; then $\omega=O\left(r^{-2}\right)$ thus 
$\nu=O\left(r^{-3}\right)$ so from Lemma 8.7 there exists a one form $\sigma^{(m)}$, solution of [60, Equation (2.4)] modulo $O\left(r^{-(m+3)}\right)$ at the right-hand-side. Set

$$
g^{(m)}:=\lambda^{(m)}\left(d t+\sigma_{i}^{(m)} d x^{i}\right)^{2}-\left(\lambda^{(m)}\right)^{-1} \gamma_{i j}^{(m)} d x^{i} d x^{j} .
$$

It then follows e.g. from [50, Section 16.2] that the Ricci tensor of the stationary spacetime metric $g^{(m)}$ has coordinate components which are $O\left(r^{-(m+3)}\right)$. By projecting on the initial data surface $\{t=0\}$ one obtains (8.10).

In order to continue, note that the collection $\mathscr{P}_{m}$ of multipole moments up to order $m$ can be viewed as an element of $\mathbb{R}^{N(m)}$, for some $N(m)$ (the exact value of which is irrelevant for our purposes); this leads to an obvious way of measuring the norm of $\mathscr{P}_{m}$.

Next, it should be clear that ten multipole moments out of the whole set $\mathscr{P}_{m}$ correspond to the global Poincaré charges of the space-time metric. For example, the $1 / r$ coefficient in the asymptotic expansion of $\lambda^{(m)}$ is related to the ADM mass of $g^{(m)}$. We denote by $\mathscr{P}^{Q}$ the relevant multipole moments, and by $\mathscr{P}_{m}^{*}$ the remaining ones, so that

$$
\mathscr{P}_{m}=\left(\mathscr{P}^{Q}, \mathscr{P}_{m}^{*}\right)
$$

We have the following:

TheOREM 8.9. - Let $m \in \mathbb{N}$ and let $\left(K_{0}, g_{0}\right)$ be a stationary solution of the vacuum Einstein equations defined on $\mathbb{R}^{3} \backslash B\left(R_{0}\right)$ with timelike $A D M$ momentum and with multipole moments up to order $m$ equal to $\mathscr{P}_{m}:=\mathscr{P}_{m}\left(K_{0}, g_{0}\right)$. There exists $\eta>0$ such that for any

$$
\left|\delta \mathscr{P}_{m}^{*}\right|<\eta
$$

there exists $\delta \mathscr{P}^{Q}$ and a smooth vacuum initial data set $(K, g)$ (not necessarily stationary) defined on $\mathbb{R}^{3} \backslash B\left(R_{0}\right)$ such that

$$
(K, g)-\left(K\left(\mathscr{P}_{m}+\delta \mathscr{P}_{m}\right), g\left(\mathscr{P}_{m}+\delta \mathscr{P}_{m}\right)\right) \in C_{\infty}^{-m-2+\varepsilon} \times C_{\infty}^{-m-1+\varepsilon}
$$

for any $\varepsilon>0$. In particular the first $m$ coefficients in an asymptotic expansion of $g$ in terms of inverse powers of $r$, and $m+1$ coefficients in that of $K$, coincide with those of the Simon-Beig approximate solution $\left(K\left(\mathscr{P}_{m}+\delta \mathscr{P}_{m}\right), g\left(\mathscr{P}_{m}+\delta \mathscr{P}_{m}\right)\right)$. An identical result holds in the class of time-symmetric initial data sets if one restricts oneself to moments associated to static space-times, provided that $K_{0} \equiv 0$.

REMARK 8.10. - The initial data set $(K, g)$ will coincide with $\left(K_{0}, g_{0}\right)$ in a neighborhood of $S\left(0, R_{0}\right)$.

Remark 8.11. - We emphasize that one is not free to choose the Poincaré charges $Q$ of the final initial data set $(K, g)$, those charges are determined by the original stationary initial data set and by the $\delta \mathscr{P}_{m}^{*}$ 's in a highly implicit manner. Further, we will have $Q-Q_{0}=O\left(\eta^{2}\right)$, where $Q_{0}$ are the Poincaré charges of $\left(K_{0}, g_{0}\right)$. 
REMARK 8.12. - It follows from the calculations of [60, Theorem 3], together with the properties of the weighted spaces in (8.14), that the "orbit space manifold" $(\mathscr{S}, \gamma)$, with $\gamma$ related to $g$ as in (8.13) with the " $(m)$ 's" removed, admits a one-point conformal compactification with a $C^{m, \alpha}$ conformally rescaled metric. In particular in the static case $(\mathscr{S}, g)$ has such a compactification.

REMARK 8.13. - Using the Schwarzschild initial data as $\left(K_{0}=0, g_{0}\right)$ one obtains a large family of static initial data with any arbitrarily prescribed finite set of small static multipole moments, except for the mass which is implicitly determined by the seed mass and the remaining multipoles. One has an obvious analogue of this result using the Kerr initial data as the reference family. Further, using Weyl metrics as $\left(K_{0}=0, g_{0}\right)$ one obtains large classes of time-symmetric initial data sets where the higher order multipoles are not necessarily small.

Proof. - The proof is essentially identical to that of Theorem 8.1. For definiteness we choose $\varepsilon=1 / 2$, the proof applies for any $0<\varepsilon<1$. Let $\mathcal{Z}_{k}^{m-1 / 2}$ denote a space of functions on $R^{3} \backslash B\left(0, R_{0}+1 / 2\right)$ which are exponentially weighted near the interior boundary $S\left(0, R_{0}+1 / 2\right)$ as in Proposition 5.10 with $t$ there equal to one, and which are weighted at infinity as in Theorem 7.7, with $\sigma$ there equal to $m-1 / 2$. (Thus, functions in $\mathcal{Z}_{k}^{m-1 / 2}$ behave as functions in $\dot{\mathscr{H}}_{k}^{m-1 / 2}$ for $r$ large.) Let $\chi \in C^{\infty}\left(\mathbb{R}^{3}\right)$ be a spherically symmetric cut-off function such that $0 \leqslant \chi \leqslant 1, \chi \equiv 1$ on $\Gamma\left(R_{0}, R_{0}+1\right)$, and $\chi \equiv 0$ on $\mathbb{R}^{3} \backslash B\left(0, R_{0}+2\right)$. Choose any $\mathscr{P}^{Q}$ satisfying

$$
\left|\mathscr{P}^{Q}\right| \leqslant \eta
$$

and on $\mathbb{R}^{3} \backslash B\left(0, R_{0}\right)$ set

$$
\begin{aligned}
g_{\delta \mathscr{P}} & =\chi g_{0}+(1-\chi) g(\mathscr{P}+\delta \mathscr{P}), \\
K_{\delta \mathscr{P}} & =\chi K_{0}+(1-\chi) K(\mathscr{P}+\delta \mathscr{P}) .
\end{aligned}
$$

We shall write $x_{\delta \mathscr{P}}$ for $\left(K_{\delta \mathscr{P}}, g_{\delta \mathscr{P}}\right), x_{0}$ for $\left(K_{0}, g_{0}\right)$, etc. Set $\varepsilon=1 / 2$, choose some $k$ large enough so that the existence and Hölder regularity results proved in the previous sections apply, it follows from (8.10) that we have

$$
\left\|J\left(x_{\delta} \mathscr{P}\right)\right\|_{\mathcal{Z}_{k+1}^{-(m-1 / 2+3)}}+\left\|\rho\left(x_{\delta} \mathscr{P}\right)\right\|_{\mathcal{Z}_{k}^{-(m-1 / 2+3)}} \leqslant C \eta .
$$

The arguments given in the proofs of Theorems 5.9 and 7.7 show that the hypotheses of Theorem 3.9 hold, and for $\eta$ small enough we obtain a solution $\delta x_{\delta \mathscr{P}} \in \mathcal{Z}_{k+2}^{-(m-1 / 2+2)} \times$ $\mathcal{Z}_{k+2}^{-(m-1 / 2+1)}$ of Equation (5.8) satisfying

$$
\left\|\delta x_{\delta} \mathscr{P}\right\|_{\mathcal{Z}_{k+2}^{-(m-1 / 2+2)} \times \mathcal{Z}_{k+2}^{-(m-1 / 2+1)}} \leqslant C \eta .
$$

Set $\delta J=J\left(x_{\delta \mathscr{P}}+\delta x_{\delta \mathscr{P}}\right), \delta \rho=\rho\left(x_{\delta \mathscr{P}}+\delta x_{\delta \mathscr{P}}\right)$. By Corollary $5.11 \delta x_{\delta \mathscr{P}}$ extends smoothly to $\mathbb{R}^{3} \backslash B\left(0, R_{0}\right)$ when extended by zero. As in the proof of Theorem 8.1 
we have

$$
\begin{aligned}
\left\langle e_{(i)}, \psi^{-2}\left(\begin{array}{c}
\delta J \\
\delta \rho
\end{array}\right)\right\rangle_{L_{\psi}^{2} \oplus L_{\psi}^{2}} & =\left\langle\left(Y_{(i)}, N_{(i)}\right), \psi^{-2}\left(\begin{array}{c}
\delta J \\
\delta \rho
\end{array}\right)\right\rangle_{L_{\psi}^{2} \oplus L_{\psi}^{2}} \\
& =\int_{\mathbb{R}^{3} \backslash B\left(0, R_{0}\right)}\left(Y_{(i)}^{j} \delta J_{j}+N_{(i)} \delta \rho\right) d \mu_{g} .
\end{aligned}
$$

We use the divergence identity (E.16) with $\left(K_{0}, g_{0}\right)$ as the background (instead of $(0, \delta)$, as was the case for Theorem 8.1$)$. The $3+1$ form of this identity reads

$$
\begin{aligned}
\left\langle e_{(i)}, \psi^{-2}\left(\begin{array}{c}
\delta J \\
\delta \rho
\end{array}\right)\right\rangle_{L_{\psi}^{2} \oplus L_{\psi}^{2}}=\lim _{R \rightarrow \infty} \int_{\{r=R\}} \mathbb{U}^{\alpha \beta}\left(x_{\delta \mathscr{P}}+\delta x_{\delta \mathscr{P}}\right) d S_{\alpha \beta} \\
-\int_{\left\{r=R_{0}\right\}} \mathbb{U}^{\alpha \beta}\left(x_{\delta} \mathscr{P}+\delta x_{\delta \mathscr{P}}\right) d S_{\alpha \beta}+O\left(\eta^{2}\right) .
\end{aligned}
$$

Now, the initial data coincide with $\left(g_{0}, K_{0}\right)$ in a neighborhood of $S\left(0, R_{0}\right)$, so that

$$
\int_{\left\{r=R_{0}\right\}} \mathbb{U}^{\alpha \beta} d S_{\alpha \beta}=0
$$

On the other hand, the limit as $R$ goes to infinity of the integral over $S(0, R)$ gives

$$
\int_{\{r=\infty\}} \mathbb{U}^{\alpha \beta} d S_{\alpha \beta}=\delta Q
$$

where $\delta Q$ is calculated from $\delta \mathscr{P}^{Q}$; we emphasize that $\delta x_{\delta} \mathscr{P}$ does not give a contribution to this integral because of the fast decay. It follows that

$$
\left\langle e_{(i)}, \psi^{-2}\left(\begin{array}{c}
\delta J \\
\delta \rho
\end{array}\right)\right\rangle_{L_{\psi}^{2} \oplus L_{\psi}^{2}}=\delta Q+O\left(\eta^{2}\right) .
$$

Let $F\left(\delta \mathscr{P}^{Q}\right)$ denote the left-hand-side of (8.18). Now, the map $\delta \mathscr{P}^{Q} \rightarrow \delta Q$ is a linear isomorphism. Further, it should be clear that $F$ is a differentiable function of $\delta \mathscr{P} Q$. The existence of a $\delta \mathscr{P}^{Q}$ such that $F\left(\delta \mathscr{P}^{Q}\right)$ vanishes can thus be inferred, for $\eta$ small enough, from the inverse function theorem. Alternatively, set

$$
G_{\eta}\left(\delta \mathscr{P}^{Q}\right)=\eta F\left(\frac{\delta \mathscr{P}^{Q}}{\eta}\right),
$$

and the existence of the required solution follows from Lemma 3.18.

One can repeat the construction of the proof with $m$ replaced by $m+1$, varying $\delta \mathscr{P}_{m+1}^{*}$ while keeping $\delta \mathscr{P}_{m}^{*}$ fixed, obtaining a finite dimensional family of distinct solutions with the same $\mathscr{P}_{m}^{*}+\delta \mathscr{P}_{m}^{*}$; this might require decreasing $\eta$. By induction, one can obtain a family of arbitrarily high dimension of distinct solutions with the same $\mathscr{P}_{m}^{*}+\delta \mathscr{P}_{m}^{*}$, for $\delta \mathscr{P}_{m}^{*}$ sufficiently small.

The above initial data are defined only on $\mathbb{R}^{3} \backslash B\left(R_{0}\right)$; however, one can now use Theorem 8.5 to construct initial data on $\mathbb{R}^{3}$, or on other asymptotically flat complete manifolds, which will coincide with the data constructed in Theorem 8.9 in the asymptotic region. 


\subsection{Space-times that are Kerrian near $\mathscr{I}^{+}$}

Space-times that are Kerrian in a neighborhood of a subset of $\mathscr{I}^{+}$are of course obtained by evolution of data which are Kerrian in a neighborhood of $i^{0}$. In some situations it might, however, be convenient to be able to construct such space-times starting directly from a hyperboloidal initial data hypersurface. It is not too difficult to adapt the original Corvino-Schoen technique to the hyperboloidal initial data setting, using the analysis above together with the relative mass identities of $[\mathbf{2 5 , 2 8}]$; this will be discussed elsewhere.

\subsection{Bondi-type asymptotic expansions at $\mathscr{I}^{+}$.}

Recall that Bondi et al. $[\mathbf{1 3}, \mathbf{5 8}]$ have proposed a set of free functions parameterising a certain asymptotic expansion of the metric at $\mathscr{I}^{+}$. It is of interest to enquire whether one can construct hyperboloidal initial data sets which would lead to spacetimes with a prescribed set of those functions. The results in Section 6 can be used to give perturbational answers to such questions, in the spirit of Theorem 8.9; this will be discussed elsewhere.

\subsection{Local and global extensions of initial data sets}

In this section ${ }^{(1)}$ we address the extension problem, that is, the following question: let us be given a vacuum initial data set $(M, K, g)$, where $\bar{M}=M \cup \partial M$ has a compact boundary $\partial M$, with the data $(K, g)$ extending smoothly, or in $C^{k}(\bar{M})$, to the boundary. Does there exist an extension across $\partial M$ of $(K, g)$ which satisfies the constraint equations? In the case where $K$ vanishes and $\partial M$ is mean convex an affirmative answer can be given by using a method ${ }^{(2)}$ due to Smith and Weinstein [61], which proceeds as follows: In a neighborhood of $\partial M$ we can write the metric in the form

$$
g=u^{2} d r^{2}+e^{2 v} \bar{\gamma}_{A B}\left(\widehat{\beta}^{A} d r+r d \theta^{A}\right)\left(\widehat{\beta}^{B} d r+r d \theta^{B}\right),
$$

where $\left(\theta^{1}, \theta^{2}\right)$ are local coordinates on $\partial M, \bar{\gamma}_{A B}$ is a fixed (independent of $r$ ) metric on $\partial M$, and $\widehat{\beta}=\widehat{\beta}^{A} \partial_{A}$ is the "shift vector". Further $r$ is a coordinate on a $\bar{M}$ neighborhood of $\partial M$ which is, say, negative and vanishes precisely on $\partial M$; to obtain (8.19) one needs further to assume that the mean extrinsic curvature $H$ of $\partial M$ has no zeros. We can extend the functions $v$ and $\widehat{\beta}^{B}$ to positive $r$ in an arbitrary way preserving their original differentiability. When $H>0$, the requirement that the

(1) A sketchy presentation of the analysis given in this section has been given in $[\mathbf{2 3}]$.

${ }^{(2)}$ Smith and Weinstein actually assume that $\partial M$ is a two-sphere, but this hypothesis is irrelevant for the discussion here. 
extended metric be Ricci-scalar flat becomes then a semi-linear parabolic equation for $u$ on $\partial M[\mathbf{6 1}]$ :

$$
r \partial_{r} u=\Gamma u^{2} \not \not u+\beta \cdot \not \supset u+A u-B u^{3},
$$

where the objects above are defined as follows: we set $\gamma_{A B}:=e^{2 v} \bar{\gamma}_{A B}$, we write $\not \Delta=r^{2} e^{2 v} \not_{\gamma}$ for the respective Laplacians of $\bar{\gamma}$ and $\gamma, \not \subset u$ is the tangential component of the gradient of $u, \Gamma=e^{-2 v} / \bar{H}, A=\bar{A} / \bar{H}$ and $B=\bar{B} / \bar{H}$, while

$$
\begin{aligned}
& \bar{\chi}=r u \chi=\left(1+r v_{r}\right) \gamma-\Pi / 2, \\
& \bar{H}=r u H=2+2 r v_{r}-e^{-2 v} \operatorname{div}_{\bar{\gamma}} \beta, \\
& \bar{A}=r \partial_{r} \bar{H}-\beta \cdot \not \bar{H}-\bar{H}+\frac{1}{2}|\bar{\chi}|_{\gamma}^{2}+\frac{1}{2} \bar{H}^{2}, \\
& \bar{B}=e^{-2 v}\left(1-\not v^{2}\right),
\end{aligned}
$$

with $\Pi=\mathcal{L}_{\widehat{\beta}} \gamma-$ the deformation tensor of $\widehat{\beta}$ and $\chi-$ the second fundamental form of the level sets of $r$. It follows from the results in [51] that Equation (8.20), with the obvious initial value, can always be solved for a small interval of $r$ 's when the initial metric and its extension are in, say, $C^{3}(\bar{M})$, obtaining a scalar-flat extension of $(M, 0, g)$. Similarly the results in [51] can be used to show that $u$ will be of class $C^{k+1}$ on the extended manifold if the remaining functions there are in $C^{2 k+1}$ (thus smooth if the initial metric is smooth up to boundary, and if the free functions above are smooth).

Our aim here is to prove two alternative extension results under smallness conditions, without the hypothesis that $K$ vanishes. Thus, assume we have a solution $(K, g) \in\left(C^{k+3, \alpha} \times C^{k+4, \alpha}\right)(\bar{M}), \alpha \in(0,1)$, of the vacuum constraints on a manifold $\bar{M}$ with compact boundary. Let $M_{0}$ be another manifold such that $\partial M_{0}$ is diffeomorphic to $\partial M$, and let $M^{\prime}$ be the manifold obtained by gluing $M$ with $M_{0}$ across $\partial M$. Let $x$ be any smooth function defined in a neighborhood $\mathscr{W}$ of $\partial M$ on $M^{\prime}$, with $\partial M=\{x=0\}$, with $d x$ nowhere vanishing on $\partial M$, and with $x>0$ on $M_{0}$. It is convenient to choose $\mathscr{V}:=\mathscr{W} \cap M_{0}$ to be diffeomorphic to $\partial M \times\left[0, x_{0}\right]$, with $x$ being a coordinate along the $\left[0, x_{0}\right]$ factor

Suppose, next, that there exists on $M_{0}$ a solution $\left(K_{0}, g_{0}\right)$ of the vacuum constraint equations which is in $\left(C^{k+3, \alpha} \times C^{k+4, \alpha}\right)\left(\bar{M}_{0}\right)$; we emphasize that we do not assume that $(K, g)$ and $\left(K_{0}, g_{0}\right)$ match across $\partial M$. We first extend $(K, g)$ to a pair $\left(K_{1}, g_{1}\right)$ defined on $M_{0}$ with the requirement that $\left(K_{1}, g_{1}\right)$ remains in $C^{k+3, \alpha} \times C^{k+4, \alpha}$; we do of course not assume that the extension is vacuum. For the purposes below it is convenient to make the extension so that $\left\|g_{1}-g_{0}\right\|_{C^{k+4, \alpha}(\mathscr{V})}+\left\|K_{1}-K_{0}\right\|_{C^{k+3, \alpha}(\mathscr{V})}$ is as small as possible. While we are not aware of an optimal prescription, a possible procedure which at least controls that norm is as follows: First, by using a partition of unity subordinate to a finite cover of a neighborhood of $\partial M_{0}$ the problem is reduced 
to that of extending functions. Given that, Corollary 3.3.2 of [2] with

$$
f_{i}=\left.\partial_{x}^{i} g\right|_{\partial M}-\left.\partial_{x}^{i} g_{0}\right|_{\partial M}, \quad i=0, \ldots, k+4,
$$

shows that there exists a $C^{k+4, \alpha}$ tensor field $f$ on $M_{0}$ such that $\partial_{x}^{i} f=f_{i}$ on $\partial M_{0}$. On $M_{0}$ we define $g_{1}$, a $C^{k+4, \alpha}$ extension of $g$, by

$$
g_{1}-g_{0}:=f,
$$

then $\partial_{x}^{i} g_{1}=\partial_{x}^{i} g$ for all $i=0, \ldots, k+4$. The proof of Lemma 3.3.1 and Corollary 3.3.2 in $[2]$ show that

$$
\left\|g_{1}-g_{0}\right\|_{C^{k+4, \alpha}(\mathscr{V})} \leqslant C \sum_{i=0}^{k+4}\left\|\left.\partial_{x}^{i} g\right|_{\partial M}-\left.\partial_{x}^{i} g_{0}\right|_{\partial M}\right\|_{C^{k+4-i, \alpha}(\partial M)} .
$$

The same procedure applies to extend $K$ to a $C^{k+3, \alpha}$ tensor field $K_{1}$ satisfying $\partial_{x}^{i} K_{1}=$ $\partial_{x}^{i} K$ for all $i=0, \ldots, k+3$ on $\partial M$, and

$$
\left\|K_{1}-K_{0}\right\|_{C^{k+3, \alpha}(\mathscr{V})} \leqslant C \sum_{i=0}^{k+3}\left\|\left.\partial_{x}^{i} K\right|_{\partial M}-\left.\partial_{x}^{i} K_{0}\right|_{\partial M}\right\|_{C^{k+3-i, \alpha}(\partial M)} .
$$

Let $\phi$ be any smooth function on $M^{\prime}$ which equals one on $M$ and on a small neighborhood $\mathscr{U} \subset \mathscr{V} \approx \partial M \times\left[0, x_{0}\right]$ of $\partial M$ in $M_{0}$, and vanishes away of $\mathscr{V}$. On $\mathscr{V}$ we set

$$
K^{\prime}=\phi K_{1}+(1-\phi) K_{0}, g^{\prime}=\phi g_{1}+(1-\phi) g_{0} .
$$

Since $J(K, g), \rho(K, g)$ vanish on $M$, while $J\left(K_{0}, g_{0}\right), \rho\left(K_{0}, g_{0}\right)$ vanish on $M_{0}$, we will have

$$
\left|\rho\left(K^{\prime}, g^{\prime}\right)\right|+\left|J\left(K^{\prime}, g^{\prime}\right)\right|_{g^{\prime}} \leqslant C\left(\left\|g_{1}-g_{0}\right\|_{C^{k+4, \alpha}(\mathscr{V})}+\left\|K_{1}-K_{0}\right\|_{C^{k+3, \alpha}(\mathscr{V})}\right) x^{k+2} ;
$$

for points at which $\phi=1$ the inequality is justified by Taylor expanding in $x$ at $\partial M$ and using the fact that $\left(K_{1}, g_{1}\right)$ satisfies the vacuum constraints on $M$; elsewhere this is justified by Taylor expanding $\rho$ and $J$ in $(K, g)$ around $\left(K_{0}, g_{0}\right)$ and using the fact that $\left(K_{0}, g_{0}\right)$ satisfies the vacuum constraints. In fact, one has

$$
\begin{aligned}
\left|\left(\nabla^{\prime}\right)^{(i)} \rho\left(K^{\prime}, g^{\prime}\right)\right|_{g^{\prime}}+ & \left|\left(\nabla^{\prime}\right)^{(i)} J\left(K^{\prime}, g^{\prime}\right)\right|_{g^{\prime}} \\
& \leqslant C\left(\left\|g_{1}-g_{0}\right\|_{C^{k+4, \alpha}(\mathscr{V})}+\left\|K_{1}-K_{0}\right\|_{C^{k+3, \alpha}(\mathscr{V})}\right) x^{k+2-i+\alpha},
\end{aligned}
$$

for all $0 \leqslant i \leqslant k+2$, with an analogous inequality holding for the Hölder quotient. So we have

$$
\begin{aligned}
& \left\|\rho\left(K^{\prime}, g^{\prime}\right)\right\|_{C_{k+2, \alpha}^{k+2+\alpha}\left(g^{\prime}, \mathscr{V}\right)}+\left\|J\left(K^{\prime}, g^{\prime}\right)\right\|_{C_{k+2, \alpha}^{k+2+\alpha}\left(g^{\prime}, \mathscr{V}\right)} \\
& \quad \leqslant C\left(\left\|g_{1}-g_{0}\right\|_{C^{k+4, \alpha}(\mathscr{V})}+\left\|K_{1}-K_{0}\right\|_{C^{k+3, \alpha}(\mathscr{V})}\right) .
\end{aligned}
$$

Assume, first, that there are no $(Y, N)$ 's such that $P^{*}(Y, N)=0$ on $\mathscr{V}$. If $\left(K_{1}, g_{1}\right)$ is sufficiently close to $\left(K_{0}, g_{0}\right)$ in $\left(C^{k+3, \alpha} \times C^{k+4, \alpha}\right)(\overline{\mathscr{V}})$ norm - equivalently, if $(K, g)$ 
and its derivatives up to appropriate order, as in (8.21)-(8.22), are sufficiently close to $\left(K_{0}, g_{0}\right)$ and its derivatives on $\partial M$, then for any $\alpha^{\prime}<\alpha$ the norm

$$
\left\|\rho\left(K^{\prime}, g^{\prime}\right)\right\|_{\dot{\mathrm{H}}_{k+2}^{k+2+\alpha^{\prime}-(n-1) / 2}\left(g^{\prime}, \mathscr{V}\right)}+\left\|J\left(K^{\prime}, g^{\prime}\right)\right\|_{\mathrm{H}_{k+2}^{k+2+\alpha^{\prime}-(n-1) / 2}\left(g^{\prime}, \mathscr{V}\right)}
$$

will also be small. If $k \geqslant\left[\frac{n}{2}\right]+1$ we can use Proposition 5.7 on $\mathscr{V}$ with $t=k+2+$ $\alpha^{\prime}-(n-1) / 2$, to conclude that there exists a solution

$$
(\delta K, \delta g) \in \mathrm{C}_{k+2, \alpha}^{k+3+\alpha^{\prime}-(n-1) / 2}\left(g^{\prime}, \mathscr{V}\right) \times \mathrm{C}_{k+2, \alpha}^{k+4+\alpha^{\prime}-(n-1) / 2}\left(g^{\prime}, \mathscr{V}\right) \subset\left(C^{k^{\prime}+2, \alpha} \times C^{k^{\prime}+2, \alpha}\right)(\overline{\mathscr{V}}),
$$

with all derivatives up to order $k^{\prime}+2$ vanishing on $\partial \mathscr{V}$, close to zero, of the vacuum constraint equations. Here $k^{\prime}$ is any integer satisfying

$$
k^{\prime} \leqslant k, k^{\prime} \leqslant k+1+\alpha^{\prime}-\alpha-(n-1) / 2<k+3 / 2-n / 2 .
$$

The above construction has a lot of if's attached, but it does provide new non-trivial extensions in the following, easy to achieve, situation:

(1) $(K, g)$ belongs to a one-parameter family of solutions $\left(K_{\lambda}, g_{\lambda}\right)$ of the vacuum constraint equations on $M$,

(2) the vacuum initial data set $\left(K_{0}, g_{0}\right)$, assumed above to be defined on $M_{0}$, arises from a vacuum initial data set defined on $M^{\prime}$, still denoted by $\left(K_{0}, g_{0}\right)$, with

(3) $\left(K_{\lambda}, g_{\lambda}\right)$ converging to $\left(\left.K_{0}\right|_{M},\left.g_{0}\right|_{M}\right)$ as $\lambda$ tends to zero in

$$
\left(C^{k+3, \alpha} \times C^{k+4, \alpha}\right)(\bar{M}) .
$$

(Replacing $M$ by a neighborhood of $\partial M$, it is of course sufficient for all the above to hold in a small neighborhood of $\partial M$.) In such a set-up, proceeding as above one obtains an extension for $\lambda$ small enough when $P^{*}$ has no kernel on $\mathscr{V}$.

The situation is somewhat more complicated when a kernel is present, though results can be obtained whenever the set-up of Theorem 3.19 applies. As an illustration, we consider a situation where $\bar{M}$ is a smooth compact submanifold, with smooth boundary, of $M^{\prime}=\mathbb{R}^{3}$. This involves no generality in the following sense: any two dimensional manifold can be embedded into $\mathbb{R}^{3}$, and so can a tubular neighborhood thereof (this will of course not be an isometric embedding in general). We allow $M$ to have more than one connected component. We will only be interested in a component of $\partial M$ which is two-sided, with one side thereof corresponding to $M$, and the other corresponding to an unbounded component of $\mathbb{R}^{3} \backslash M$ (we assume that such a component exists). A component of $\partial M$ with this property will be called an exterior boundary, and will be denoted by $\partial_{\text {ext }} M$. We assume $K_{0} \equiv 0$, and we let $g_{0}$ be the Euclidean metric on $\mathbb{R}^{3}$. Replacing $\bar{M}$ by a tubular neighborhood $\left(-x_{0}, 0\right] \times \partial_{\text {ext }} M$ we can thus identify $\bar{M}$ with a subset of $\mathbb{R}^{3}$. We note that the closure of $\bar{M}$ in $\mathbb{R}^{3}$ will then have a boundary with two components, $\left\{-x_{0}\right\} \times \partial_{\text {ext }} M$ and $\{0\} \times \partial_{\text {ext }} M$, but we will ignore $\left\{-x_{0}\right\} \times \partial_{\text {ext }} M$ if occurring, and consider only $\{0\} \times \partial_{\text {ext }} M$, which is the exterior boundary of the new $M$. From now on we write $\partial M$ for $\{0\} \times \partial_{\text {ext }} M$. 
We assume that $(K, g)$ are close to $\left(K_{0}, g_{0}\right)$ :

$$
\left\|g-g_{0}\right\|_{C^{k+4, \alpha}(\bar{M})}+\left\|K-K_{0}\right\|_{C^{k+3, \alpha}(\bar{M})}<\varepsilon ;
$$

such metrics can be constructed by the conformal method. We now repeat the construction of the proof of Theorem 8.1 with $R=1$ there, so that no rescalings of the metrics are performed. Equation (8.6) becomes

$$
\begin{aligned}
\left\langle e_{(i)}, \psi^{-2}\left(\begin{array}{c}
\delta J \\
\delta \rho
\end{array}\right)\right\rangle_{L_{\psi}^{2} \oplus L_{\psi}^{2}}= & \int_{\left\{x_{0}\right\} \times \partial M} \mathbb{U}^{\alpha \beta}\left(x_{Q, R}+\delta x_{Q, R}\right) d S_{\alpha \beta} \\
& -\int_{\{0\} \times \partial M} \mathbb{U}^{\alpha \beta}\left(x_{Q, R}+\delta x_{Q, R}\right) d S_{\alpha \beta}+O\left(\varepsilon^{2}\right),
\end{aligned}
$$

We set

$$
Q_{(i)}^{0}:=\int_{\{0\} \times \partial M} \mathbb{U}^{\alpha \beta} d S_{\alpha \beta} .
$$

It follows from (8.23) that there exist a constant $C>0$ such that $\left|Q^{0}\right| \leqslant C \varepsilon$. We restrict ourselves to $Q$ 's such that

$$
\left|Q-Q^{0}\right| \leqslant \varepsilon \Longrightarrow|Q| \leqslant(1+C) \varepsilon .
$$

Then

$$
\int_{\left\{x_{0}\right\} \times \partial M} \mathbb{U}^{\alpha \beta} d S_{\alpha \beta}=Q_{(i)}+O\left(\varepsilon^{2}\right),
$$

for $\varepsilon$ small enough. We are thus led to

$$
\left\langle e_{(i)}, \psi^{-2}\left(\begin{array}{c}
\delta J \\
\delta \rho
\end{array}\right)\right\rangle_{L_{\psi}^{2} \oplus L_{\psi}^{2}}=Q_{(i)}-Q_{(i)}^{0}+O\left(\varepsilon^{2}\right) .
$$

For $\varepsilon$ small enough one would like to conclude as before. There is, however, a difficulty which arises here because the map of Proposition F.3 degenerates at $m=0$, as is made clear by the need of dividing by $m$ in (F.6) when one wishes to determine $a_{i}$ from $J_{0 i}$. This leads to further conditions if one wishes the argument to go through: roughly speaking, one needs to assume that $m$ is of order of $\varepsilon$, that the ratio $|\vec{p}| / m$ is strictly bounded away from one, and that the ratio $J_{\mu \nu} / m$ is $o(\varepsilon)$; if that is the case, we can use the Lemma 3.18 with $U=V=B(0,1), x=\left(Q-Q^{0}\right) / \varepsilon, \lambda=1 / \varepsilon$, $G_{\lambda}(x)=\frac{1}{\varepsilon}\left(Q-Q^{0}+O\left(\varepsilon^{2}\right)\right)=x+O(\varepsilon)$ and $y=0$ to conclude. Rather than making general statements along those lines, with hypotheses which appear difficult to control, we shall assume that the antipodal map

$$
x^{i} \longrightarrow-x^{i}
$$

preserves $g$ and maps $K$ to $-K$; clearly $\left(K_{1}, g_{1}\right)$ can be constructed as to preserve this property, and we will only consider such extensions. Such data will be referred to 
as parity-covariant. ${ }^{(3)}$ Nontrivial parity covariant initial data $(K, g)$, as close to the Euclidean metric as one wishes, can be easily constructed by the conformal method - we do this, for completeness, in Appendix H.

Assume, first, that $K$ - and hence $K_{1}$ - vanishes. Now, the construction of Theorem 5.6 preserves all symmetries of initial data, so that gluing together "up to kernel" $g_{1}$ with (neither boosted nor translated) Schwarzschild metrics $g_{m}$ will lead to sets $\left(K_{1}+\delta K_{Q}=0, g_{1}+\delta g_{Q}\right)$ still being covariant under the antipodal map (8.26). One then obtains, by parity considerations,

$$
J_{\mu \nu}\left(K_{1}+\delta K_{Q}, g_{1}+\delta g_{Q}\right)=0
$$

similarly, the left-hand side of (8.25) vanishes for those projections which are associated with the $J_{\mu \nu}$ 's. Then, the only possibly non-zero component of the projection on the kernel is the one which corresponds to the mass. In that case no difficulties with the crossing of $m=0$ arise, and we can use on $M_{\text {ext }}$ the family of Schwarzschild metrics $g_{m}$ with $m \in(-\delta, \delta)$, with any $\delta \leqslant \min (1,1 / R)$. Rather than invoking the Brouwer fixed point theorem we note that if the reference Schwarzschild metric $g_{m}$ has mass $m=-\min (C \varepsilon, \delta)$ we obtain ${ }^{(4)}$ a strictly negative value of the projection (8.25) when $\varepsilon$ is small enough. The value $m=m_{0}+\varepsilon$ leads to a strictly positive value of the projection in (8.25) (decreasing $\varepsilon$ if necessary); since the left-hand-side of (8.25) depends continuously upon $m$ there exists $m \in\left(-C \varepsilon, m_{0}+\varepsilon\right)$ such that the left-hand side of (8.25) vanishes.

The case of non-vanishing parity-antisymmetric $K$ 's is handled as follows: let $0 \leqslant$ $\lambda<1$ and consider the set of $(K, g)$ satisfying

$$
\left|\overrightarrow{p_{0}}\right|_{\delta} \leqslant \lambda m_{0} .
$$

Equation (8.27) still holds, so that the only projections on the kernel which are nonzero are those associated with the mass and the momentum. Since the charges in (8.25) are smaller than $\varepsilon$, while the error term is one order higher, an argument along lines similar to those of Lemma 3.18 gives existence of a solution when $\varepsilon$ is small enough

Summarising, we have proved:

\footnotetext{
(3) One of the purposes of the parity conditions here is to ensure vanishing of the centre of mass. This last property also holds when both $g$ and $K$ are even. However, for even $K$ and small $m$ there arise some difficulties with non-zero angular momentum, essentially identical to those of non-zero centre of mass; see Section 8.9 for an analysis of one such example.

(4) At this stage one could use harmonic coordinates, and invoke the small data calculations of Bartnik [7] to conclude that the mass $m_{0}$ as defined by $Q^{0}$ must be positive, so that restricting oneself to the family of Schwarzschild metrics with $m \geqslant 0$ suffices. However, this is not necessary, and positivity of $m_{0}$ is actually a consequence of the positive energy theorem and of our argument here, regardless of the coordinate systems used, for data close enough to Minkowski ones.
} 
THEOREM 8.14. - Let $k \geqslant\left[\frac{n}{2}\right]+3$, and let $k^{\prime}$ be the largest integer strictly smaller than $k+(3-n) / 2$. Consider parity-covariant vacuum initial data sets $(K, g) \in$ $C^{k+2} \times C^{k+3}$ on a compact smooth submanifold $\bar{M}$ of $\mathbb{R}^{3}$ such that (8.28) is satisfied with some $0 \leqslant \lambda<1$, and let $\Omega$ be any parity invariant bounded domain with smooth boundary containing $\bar{M}$. If (8.23) holds with some $\varepsilon>0$ small enough, then there exists a vacuum $C^{k^{\prime}} \times C^{k^{\prime}}$ extension of $(K, g)$ across the exterior component of $\partial M$, with the extension being a (perhaps boosted) Schwarzschild solution outside of $\Omega$.

Identical results can be similarly obtained when the source fields $\rho$ and $J$ are prescribed a priori, rather than arising from some field theoretical model which has its own constraint equations. It is also clear that the arguments generalize to EinsteinMaxwell electro-vacuum constraint equations, though we did not attempt to carry through the details of such a construction.

\subsection{Localized Isenberg-Mazzeo-Pollack gluings}

In important recent papers, Isenberg, Mazzeo and Pollack have introduced a conformal gluing method for initial data sets $[\mathbf{4 5}, \mathbf{4 6}]$; this generalizes previous work of Joyce $[\mathbf{4 7}]$ which treats the purely Riemannian case. The problem addressed is the following: let $(M, K, g)$ be a vacuum initial data set on a not-necessarily connected manifold $M$; for simplicity we assume in this section that all the fields are smooth, though the results below can be stated under finite differentiability conditions. One also assumes that either $M$ is compact, or $(M, K, g)$ is asymptotically Euclidean, or $(M, K, g)$ is asymptotically hyperboloidal; on any compact component a non-degeneracy condition has moreover to be imposed. Let $p_{i} \in M, i=1,2$, and for $t$ small let $\widehat{M}_{t}$ be a manifold obtained by cutting from $M$ two geodesic balls $B\left(p_{i}, t\right)$ of radius $t$ centred at $p_{i}$, and gluing the left-over manifolds by adding a neck. It is shown in [46] that when $\operatorname{tr} K$ is constant over the $B\left(p_{i}, t\right)$ 's, then one can construct a one-parameter family of new initial data sets $\left(\widehat{M}_{t}, \widehat{K}_{t}, \widehat{g}_{t}\right), t \in\left(0, t_{0}\right)$ with the property that $\left(K_{t}, g_{t}\right)$ converges uniformly, in any $C^{k, \alpha}$ norm, on any compact subset of $M \backslash\left\{p_{1}, p_{2}\right\}$, to $(K, g)$. In fact, $\left(\widehat{K}_{t}, \widehat{g}_{t}\right)$ are conformal deformations of $(K, g)$ on $M \backslash\left(B\left(p_{1}, t_{1}\right) \cup B\left(p_{2}, t_{1}\right)\right)$ for $t<t_{1}$. The technique will be referred to as the IMP gluing.

Let us show that in generic situations the gluing can be performed so that the new initial data coincide with the original ones away from a small neighborhood of the $p_{i}$ 's:

THEOREM 8.15. - Let $t_{0}$ be small enough so that the geodesic spheres $S\left(p_{i}, t\right)$ are smooth manifolds for $t \leqslant 2 t_{0}$. Suppose that there exists $0<t_{1} \leqslant t_{0}$ such that the set of $K I D s$ on $\Gamma_{p_{i}}\left(t_{1}, 2 t_{1}\right):=B\left(p_{i}, 2 t_{1}\right) \backslash \overline{B\left(p_{i}, t_{1}\right)}$ is trivial. Then there exists $t_{2} \leqslant t_{1}$ 
and a family of smooth vacuum initial data sets $\left(\widehat{M}_{t}, \widetilde{K}_{t}, \widetilde{g}_{t}\right), t \leqslant t_{2}$ such that

$$
\left(\widetilde{K}_{t}, \widetilde{g}_{t}\right)=(K, g) \text { on } M \backslash\left(B\left(p_{1}, 2 t_{1}\right) \cup B\left(p_{2}, 2 t_{1}\right)\right) \text {. }
$$

Moreover, $\left(\widetilde{K}_{t}, \widetilde{g}_{t}\right)$ approaches $(K, g)$ uniformly as $t \rightarrow 0$ on any compact subset of $B\left(p_{i}, 2 t_{1}\right) \backslash\left\{p_{i}\right\}$

REMARK 8.16. - For generic metrics the set of KID's on $\Gamma_{p_{i}}\left(t_{1}, 2 t_{1}\right)$ will be trivial for all $t_{1}$.

REMARK 8.17. - The initial data set $\left(\widetilde{K}_{t}, \widetilde{g}_{t}\right)$ will coincide with the IMP data set $\left(\widehat{K}_{t}, \widehat{g}_{t}\right)$ in the neck region.

REMARK 8.18. - Suppose that $M$ has two connected components $M_{1}$ and $M_{2}$, with each of the $p_{i}$ 's lying in a different component, say $p_{1} \in M_{1}$ and $p_{2} \in M_{2}$. If the set of KIDs on $\Gamma_{p_{1}}\left(t_{1}, 2 t_{1}\right)$ is trivial, then the construction below clearly gives $\left(\widetilde{K}_{t}, \widetilde{g}_{t}\right)=$ $(K, g)$ on $M_{1} \backslash B\left(p_{1}, 2 t_{1}\right)$ for $t \leqslant t_{2}$, regardless of whether or not there are KIDs on annuli on the other component.

Proof. - Let $\chi$ be a positive smooth radial cut-off function equal to 1 in a neighborhood of $t_{1}$ and equal to zero in a neighborhood of $2 t_{1}$. On $\Gamma_{p_{1}}\left(t_{1}, 2 t_{1}\right)$ set

$$
\begin{aligned}
\stackrel{\circ}{K}_{t} & =\chi \widehat{K}_{t}+(1-\chi) K, \\
\stackrel{\circ}{g}_{t} & =\chi \widehat{g}_{t}+(1-\chi) g .
\end{aligned}
$$

Then $\left(\stackrel{\circ}{K}_{t}, \stackrel{\circ}{g}_{t}\right)$ coincides with the IMP data $\left(\widehat{K}_{t}, \widehat{g}_{t}\right)$ in a neighborhood of $S\left(p_{1}, t_{1}\right)$, and coincides with the original data $(K, g)$ in a neighborhood of $S\left(p_{1}, 2 t_{1}\right)$. It follows that $\rho\left(\stackrel{\circ}{K}_{t}, \stackrel{\circ}{g}_{t}\right)$ and $J\left(\stackrel{\circ}{K}_{t}, \stackrel{\circ}{g}_{t}\right)$ are supported away from the boundary in $\Gamma_{p_{1}}\left(t_{1}, 2 t_{1}\right)$. Since the IMP data converge uniformly to the original ones on $\Gamma_{p_{1}}\left(t_{1}, 2 t_{1}\right)$ we will have

$$
\lim _{t \rightarrow 0} \rho\left(\stackrel{\circ}{K}_{t}, \stackrel{\circ}{g}_{t}\right)=0=\lim _{t \rightarrow 0} J\left(\stackrel{\circ}{K}_{t}, \stackrel{\circ}{g}_{t}\right) \text {. }
$$

Theorem 5.9 and Corollary 5.11 provides $0<t_{2} \leqslant t_{1}$ such that for all $0<t \leqslant t_{2}$ there exists a solution $\left(\stackrel{\circ}{K}_{t}, \stackrel{\circ}{g}_{t}\right)$ of the vacuum constraint equations which is smoothly extended by $\left(\widehat{K}_{t}, \widehat{g}_{t}\right)$ across $S\left(p_{1}, t_{1}\right)$ and by $(K, g)$ across $S\left(p_{1}, 2 t_{1}\right)$, as desired.

\subsection{Vacuum space-times with a smooth global $\mathscr{I}$}

The results proved so far can be used to establish existence of a reasonably large class of small-data, vacuum space-times with a global smooth $\mathscr{I}$. While we refer the reader to $[\mathbf{2 3}]$ for the overall details of this construction, we note the following here: first, in [23] we did not claim that the resulting space-times will have a smooth $\mathscr{I}$, as we did not realize by then ${ }^{(5)}$ that Corollary 5.11 holds. We note that the argument of Theorem 8.14 does not seem to work with $k=\infty$. However, for the construction of the space-times with a smooth $\mathscr{I}^{+}$one can proceed as follows: in the setting of

${ }^{(5)}$ We are grateful to J. Corvino for pointing out that Corollary to us. 
the proof of Theorem 8.14, choose some $k$ large enough so that the previous existence and regularity results apply, let $g_{1}$ be an extension as in (8.21). A small smoothing will lead to an extension which is $C^{\infty}$. One then continues the construction as in Section 8.6 using an exponentially weighted Sobolev space, where the exterior region has been slightly increased, so that its boundary has been moved from $\partial M$ to the set $\{x=-\varepsilon\}$, for some small positive $\varepsilon$. The remaining arguments remain unchanged. Instead of obtaining a smooth extension of the initial data on $M$ one will have a smooth extension of the initial data on $M \backslash\{-\varepsilon \leqslant x \leqslant 0\}$, but this difference is irrelevant for the purpose of constructing some examples.

In Theorem 8.14 we have used the family of boosted Schwarzschild metrics in the exterior region. It should be clear that any parity-covariant reference family of stationary metrics can be used there. This, together with arguments identical to those of [23], establishes existence of asymptotically simple parity-covariant space-times which are stationary near $i^{0}$, with metrics which are not necessarily Schwarzschild near $i^{0}$.

A generic metric so constructed will have no KID's. Whenever that occurs, we can use the conformal method to slightly deform the initial data on $B(0, R)$ so that the new initial data are not parity symmetric, and then use Theorem 5.9 and Corollary 5.11 to obtain perturbed initial data on $B(0, R+1)$ which will not satisfy any parity conditions, and which will coincide with the starting ones on $\mathbb{R}^{3} \backslash B(0, R)$. Making all perturbations small enough one will obtain a maximal globally hyperbolic development with a global $\mathscr{I}$, and with a metric which does not satisfy any parity conditions. In particular asymptotically simple space-times which are Kerrian near $i^{0}$, with non-vanishing angular momentum, can be obtained in this way.

\section{9. "Many Kerr" initial data}

A noteworthy application of the techniques of Section 8.6 is the construction of initial data containing black-hole regions with exactly Kerrian geometry both near the apparent horizons, and in the asymptotic region. This generalizes a construction of $[\mathbf{2 3}]$, which leads to "many Schwarzschild" black holes. More precisely, let $I \in \mathbb{N}$, we will construct initial data for a vacuum space-time with the following properties:

(1) There exists a compact set $\mathscr{K}$ such that $(K, g)$ are initial data for a Kerr metric with some mass parameter $m$ and some angular momentum parameter $a$ on each connected component of $M \backslash \mathscr{K}$ (in general different $(m, a)$ 's for different components);

(2) let $\mathcal{S}$ denote the usual marginally trapped sphere within the Carter extension of the Kerr solution, then $M$ contains $I$ such surfaces, with the space-time metric being exactly a Kerr metric in a neighborhood of each $\mathcal{S}$.

In fact, $(M, g)$ will be obtained by gluing together $I$ Kerr initial data with small masses. The resulting space-time $(M, g)$ can be thought of as having $I$ black holes: 
Indeed, the results in $[\mathbf{2 7}]$ show that for several configurations the intersection of the black hole region in the associated maximal globally hyperbolic development of the initial data with the Cauchy surface will have at least $I$ connected components.

Let us pass to the construction: Let $N$ be the integer part of $I / 2$, choose two strictly positive radii $0<4 R_{1}<R_{2}<\infty$, and for $i=1, \ldots, 2 N$ let the points

$$
\vec{x}_{i} \in \Gamma_{0}\left(4 R_{1}, R_{2}\right):=B\left(0, R_{2}\right) \backslash \overline{B\left(0,4 R_{1}\right)}
$$

$\left(B(\vec{a}, R)\right.$ - open coordinate ball centred at $\vec{a}$ of radius $R$ ) and the radii $r_{i}$ be chosen so that the balls $B\left(\vec{x}_{i}, 4 r_{i}\right)$ are pairwise disjoint, all included in $\Gamma_{0}\left(4 R_{1}, R_{2}\right)$. Set

$$
\Omega:=\Gamma_{0}\left(R_{1}, R_{2}\right) \backslash\left(\cup_{i} \overline{B\left(\vec{x}_{i}, r_{i}\right)}\right) .
$$

We shall further assume that $\Omega$ is invariant under the parity map $\vec{x} \rightarrow-\vec{x}$. Let

$$
\vec{Q}=\left((m, a, \vec{\omega}),\left(m_{0}, a_{0}, \vec{\omega}(0)\right),\left(m_{1}, a_{1}, \vec{\omega}(1)\right), \ldots,\left(m_{2 N}, a_{2 N}, \vec{\omega}(2 N)\right)\right.
$$

be a set of numbers and unit vectors satisfying $2 m<R_{1}, 2 m_{0}<R_{1}, 2 m_{i}<r_{i}$. If $I=2 N$ we require $a_{0}=m_{0}=0$. Whenever one of the $a$ 's is zero the associated vector $\vec{\omega}$ is irrelevant, and then we forget it altogether. Let $\left(K_{\vec{Q}}, g_{\vec{Q}}\right)$ be constructed as follows:

(1) If $I=2 N+1$ then on $\Gamma_{0}\left(R_{1}, 2 R_{1}\right)$ the initial data $\left(K_{\vec{Q}}, g_{\vec{Q}}\right)$ are the initial data for a Kerr metric with mass $m_{0}$, with angular momentum $a_{0} m_{0} \vec{\omega}(0)$, centred at 0 ; here

$$
\begin{aligned}
& a_{0} m_{0} \omega(0)^{\ell}:=\frac{1}{8 \pi} \lim _{R \rightarrow \infty} \int_{S(0, R)} \varepsilon_{j k}^{\ell} x^{j}\left((\operatorname{tr} K) g^{k l}-K^{k l}\right) d S_{l} \\
&= \frac{1}{8 \pi} \int_{S\left(0, R_{1}\right)} \varepsilon_{j k}^{\ell} x^{j}\left((\operatorname{tr} K) g^{k l}-K^{k l}\right) d S_{l} \\
&+\int_{\mathbb{R}^{3} \backslash B\left(0, R_{1}\right)} \nabla_{l}\left(\varepsilon^{\ell}{ }_{j k} x^{j}\right)\left((\operatorname{tr} K) g^{k l}-K^{k l}\right) \\
&= \frac{1}{8 \pi} \int_{S\left(0, R_{1}\right)} \varepsilon_{j k}^{\ell} x^{j}\left((\operatorname{tr} K) g^{k l}-K^{k l}\right) d S_{l} \\
&+O\left(m_{0} a_{0}\left(a_{0}^{2}+m_{0}+a_{0}^{2} m_{0}\right)\right) .
\end{aligned}
$$

In the third line of (8.30) the covariant derivative $\nabla_{l}\left(\varepsilon^{\ell}{ }_{j k} x^{j}\right)$ is understood as that of a vector field with vector index $k$, at $\ell$ fixed. (To obtain the estimate for the error term we are using Boyer-Lindquist coordinates as discussed in Appendix F. Recall that $K$ is a linear combination of space-covariant derivatives of (F.7), which leads to $K=O\left(a_{0} m_{0}\right)$. Next, (F.1) gives the estimate $\Gamma^{i}{ }_{j k}=O\left(a_{0}^{2}+m_{0}+a_{0}^{2} m_{0}\right)$ for the space Christoffel symbols in asymptotically Euclidean coordinates, leading to (8.30).) If $I=2 N$ then we take $\left(K_{\vec{Q}}, g_{\vec{Q}}\right)=(0, \delta)$ on $\Gamma_{0}\left(R_{1}, 2 R_{1}\right)$;

(2) on $\Gamma_{0}\left(3 R_{1}, R_{2}\right) \backslash\left(\cup_{i} \overline{B\left(\vec{x}_{i}, 4 r_{i}\right)}\right)$ the initial data $\left(K_{\vec{Q}}, g_{\vec{Q}}\right)$ are the initial data for a Kerr metric with mass $m$, with angular momentum $a m \vec{\omega}$, centred at 0 . As 
in (8.30) we have

$$
a m \omega^{\ell}=\frac{1}{8 \pi} \int_{S\left(0, R_{2}\right)} \varepsilon^{\ell}{ }_{j k} x^{j}\left((\operatorname{tr} K) g^{k l}-K^{k l}\right) d S_{l}+O\left(m a\left(a^{2}+m+a^{2} m\right)\right) ;
$$

(3) on $\Gamma_{0}\left(2 R_{1}, 3 R_{1}\right)$ the tensor fields $\left(K_{\vec{Q}}, g_{\vec{Q}}\right)$ interpolate between the two Kerr initial data already defined above using a usual cut-off function;

(4) on the annuli $\Gamma_{\vec{x}_{i}}\left(r_{i}, 2 r_{i}\right):=B\left(\vec{x}_{i}, 2 r_{i}\right) \backslash \overline{B\left(\vec{x}_{i}, r_{i}\right)}$ the initial data $\left(K_{\vec{Q}}, g_{\vec{Q}}\right)$ are the initial data for a Kerr metric with mass $m$, with angular momentum $a_{i} m_{i} \vec{\omega}(i)$, centred at $\vec{x}_{i}$. The vanishing of the total momentum of the Kerr metric implies

$$
\begin{aligned}
a_{i} m_{i} \omega(i)^{\ell} & :=\frac{1}{8 \pi} \lim _{R \rightarrow \infty} \int_{S\left(\vec{x}_{i}, R\right)} \varepsilon^{\ell}{ }_{j k}\left(x^{j}-x_{i}^{j}\right)\left((\operatorname{tr} K) g^{k l}-K^{k l}\right) d S_{l} \\
= & \frac{1}{8 \pi} \lim _{R \rightarrow \infty} \int_{S\left(\vec{x}_{i}, R\right)} \varepsilon^{\ell}{ }_{j k} x^{j}\left((\operatorname{tr} K) g^{k l}-K^{k l}\right) d S_{l} \\
= & \frac{1}{8 \pi} \int_{S\left(\vec{x}_{i}, r_{i}\right)} \varepsilon^{\ell}{ }_{j k} x^{j}\left((\operatorname{tr} K) g^{k l}-K^{k l}\right) d S_{l} \\
& +O\left(m_{i} a_{i}\left(a_{i}^{2}+m_{i}+a_{i}^{2} m_{i}\right)\right) ;
\end{aligned}
$$

(5) on the annulus $\Gamma_{\vec{x}_{i}}\left(2 r_{i}, 3 r_{i}\right)$ the tensor fields $\left(K_{\vec{Q}}, g_{\vec{Q}}\right)$ interpolate between the initial data already defined above using a usual cut-off function;

(6) all the parameters are so chosen, and the gluings are so performed, that the resulting initial data set is symmetric under the parity map $\vec{x} \rightarrow-\vec{x}$; note that the Kerr initial data are exactly parity symmetric in the Boyer-Lindquist coordinates, compare the discussion at the end of Appendix F.

Clearly $g_{\vec{Q}=0}$ is the flat Euclidean metric on $\Omega$, in particular it is vacuum. For $|\vec{Q}| \leqslant 1$ this implies

$$
\left|\rho\left(K_{\vec{Q}}, g_{\vec{Q}}\right)\right| \leqslant C|\vec{Q}|
$$

By construction we also have

$$
\left|J\left(K_{\vec{Q}}, g_{\vec{Q}}\right)\right| \leqslant C|\vec{Q}|\left(|a|+\sum_{i=0}^{2 N}\left|a_{i}\right|\right) .
$$

Similar inequalities hold for derivatives of $J$ and $\rho$.

Suppose that

$$
|\vec{Q}| \leqslant \delta ;
$$

Theorem 5.9 and Corollary 5.11 show that there exists $0<\delta \leqslant 1$ such that for all $Q$ satisfying (8.33) there exists a set of $C^{\infty}$ tensor fields $\left(\widehat{K}_{\vec{Q}}, \widehat{g}_{\vec{Q}}\right)$ defined on $\Omega$ which agrees with $\left(K_{\vec{Q}}, g_{\vec{Q}}\right)$ in a neighborhood of $\partial \Omega$, and which satisfies the constraint equations except for the projection on the kernel of $P^{*}$. (Here one should use Theorem 5.9 on a domain strictly included in the interior of $\Omega$; a similar comment applies whenever we are referring to that theorem below.) Uniqueness implies that the solution will be 
even. Parity shows that both the centre of mass and the total momentum vanish, so that the obstruction is the non-vanishing of the four integrals

$$
\begin{gathered}
\frac{1}{8 \pi} \int_{\Omega} \rho\left(\widehat{K}_{\vec{Q}}, \widehat{g}_{\vec{Q}}\right)=m-\sum_{i=0}^{2 N} m_{i}+O\left(\delta^{2}\right), \\
\frac{1}{8 \pi} \int_{\Omega} \varepsilon^{\ell}{ }_{j k} x^{j} J^{k}\left(\widehat{K}_{\vec{Q}}, \widehat{g}_{\vec{Q}}\right)=a m \omega^{\ell}-\sum_{i=0}^{2 N} a_{i} m_{i} \omega(i)^{\ell}+O\left(\delta^{2}\right) .
\end{gathered}
$$

Now, one would like to apply a fixed point theorem to conclude the existence of a solution, but this does not seem to work directly because the error term in (8.34b) is too large. Instead, we proceed as follows: Suppose, first, that

$$
a=a_{0}=a_{1}=\cdots=a_{2 N}=0,
$$

and write $g_{\vec{M}}$ and $\widehat{g}_{\vec{M}}$ for the resulting $g_{\vec{Q}}$ and $\widehat{g}_{\vec{Q}}$. We then have $K_{\vec{Q}}=\widehat{K}_{\vec{Q}}=0$, so that the left-hand-side of (8.34b) vanishes identically. Fix any set of $m_{i}$ 's, $i=$ $0, \ldots, 2 N$, satisfying

$$
\sum_{i=0}^{2 N}\left|m_{i}\right| \leqslant \delta / 4
$$

If $\delta$ is small enough the right-hand-side of (8.34a) with $m=\delta / 2$ will be strictly positive; it will be strictly negative with $m=-\delta / 2$, by continuity there exists $m$ such that $\widehat{g}_{\vec{M}}$ will be Ricci scalar flat.

To continue, suppose that the $\vec{x}_{i}$ 's have $m_{i} \geqslant 0$, with at least one $m_{i}>0$, and they are not aligned. Then the vacuum initial data set $\left(0, \widehat{g}_{\vec{M}}\right)$ on $\Omega$ has no KIDs. ${ }^{(6)}$ We can therefore use Theorem 5.9 and Corollary 5.11 around $\left(0, \widehat{g}_{\vec{M}}\right)$ to construct an initial data set $(g, K)$, which coincides with $\left(K_{\vec{Q}}, g_{\vec{Q}}\right)$ near $\partial \Omega$, for any collection of $a$ 's and $\vec{\omega}$ 's satisfying

$$
|a|+\sum_{i=0}^{2 N}\left|a_{i}\right|<\varepsilon,
$$

when $\varepsilon$ is small enough. For further purposes we impose $\varepsilon \leqslant \delta$.

Suppose, finally, that all the $\vec{x}_{i}$ 's are aligned along an axis, say the $z$-axis. Then the vacuum initial data set $\left(0, \widehat{g}_{\vec{M}}\right)$ on $\Omega$ has exactly one $\operatorname{KID}(Y, 0)$, where $Y$ is the Killing vector associated with the rotations around the $z$-axis. We repeat now the previous construction, with the following difference: on $\Gamma_{0}\left(R_{1}, R_{2}\right) \backslash\left(\cup_{i} \overline{B\left(\vec{x}_{i}, r_{i}\right)}\right)$ we use the Ricci scalar flat metric $\widehat{g}_{\vec{M}}$, and in points (3) and (5) above $\widehat{g}_{\vec{Q}}$ is taken as

\footnotetext{
${ }^{(6)}$ In the case $K=0$ the KID equations decouple, so if $(N, Y)$ is a $\mathrm{KID}$, then so are $(0, N)$ and $(Y, 0)$. The existence of a KID with $Y=0$ would lead to a vacuum static space-time with a non-connected black hole with all horizons non-degenerate, which is not possible by [14]. Thus $N=0$. By [10] the only remaining possibility is a single Killing vector field $Y$ which is a non-trivial rotation in the region where the metric is Schwarzschild, which is clearly only possible if all the $\vec{x}_{i}$ 's are aligned.
} 
a combination with cut-off functions of the relevant Kerr metric and of $\widehat{g}_{\vec{M}}$. Assuming (8.35), we will have

$$
\left\|\widehat{g}_{\vec{Q}}-\widehat{g}_{\vec{M}}\right\|_{C_{k}} \leqslant C(k) \varepsilon, \quad\left\|\widehat{K}_{\vec{Q}}\right\|_{C_{k}} \leqslant C(k) \varepsilon
$$

We need a somewhat more precise version of the calculation in (8.30). By hypothesis the vector field $Y_{k}:=\varepsilon_{k l}^{z} x^{k}=\partial / \partial \varphi$ is a Killing vector of the metric $g_{\vec{M}}$, so that

$$
\nabla_{k} Y_{l}+\nabla_{l} Y_{k}=\stackrel{\circ}{\nabla}_{k} Y_{l}+\stackrel{\circ}{\nabla}_{l} Y_{k}+2 C_{k l}^{r} Y_{r}=2 C_{k l}^{r} Y_{r},
$$

where $\nabla^{\circ}$ is the covariant derivative of $g_{\vec{M}}$, while $C_{k l}^{r}$ is the difference of the Christoffel symbols of $g_{\vec{Q}}$ and $g_{\vec{M}}$. It follows that

$$
\nabla_{k} Y_{l}+\nabla_{l} Y_{k}=O(\varepsilon) .
$$

Applying the divergence theorem on $\mathbb{R}^{3} \backslash\left(\cup_{i} B\left(\vec{x}_{i}, r_{i}\right) \cup B\left(0, R_{1}\right)\right)$ as in the second and third lines of (8.30), using the last two lines of (8.30) together with (8.31) and (8.32) we therefore obtain

$$
\frac{1}{8 \pi} \int_{\Omega} \varepsilon^{\ell}{ }_{j k} x^{j} J^{k}\left(\widehat{K}_{\vec{Q}}, \widehat{g}_{\vec{Q}}\right)=a m \omega^{\ell}-\sum_{i=0}^{2 N} a_{i} m_{i} \omega(i)^{\ell}+O\left(\varepsilon^{2}+\varepsilon \delta^{2}\right) .
$$

Here the $\rho$ integral, as well as the integrals (8.37) with $\ell=x$ and $\ell=y$ are already identically zero, so that the only remaining obstruction is the integral at the lefthand-side of (8.37) with $\ell=z$. We choose the exterior Kerr solution so that $\omega^{z} \neq 0$. At this stage we might need to decrease $\delta$ to conclude, so we suppose that we are working in a family of mass parameters $\left(m, m_{i}\right)$ so that $\delta / m$ is uniformly bounded from above independently of $m$ (in particular $m$ is not zero). This gives

$$
\frac{1}{8 \pi m \omega^{z}} \int_{\Omega} \varepsilon^{z}{ }_{j k} x^{j} J^{k}\left(\widehat{K}_{\vec{Q}}, \widehat{g}_{\vec{Q}}\right)=a-\sum_{i=0}^{2 N} a_{i} \frac{m_{i}}{m} \frac{\omega(i)^{z}}{\omega^{z}}+O\left(\frac{\varepsilon^{2}}{\delta}+\varepsilon \delta\right) .
$$

We can choose $0<\varepsilon^{\prime}<\varepsilon / 2$ so that if

$$
\sum_{i=0}^{2 N}\left|a_{i}\right|<\varepsilon^{\prime}
$$

then

$$
\left|\sum_{i=0}^{2 N} a_{i} \frac{m_{i}}{m} \frac{\omega(i)^{z}}{\omega^{z}}\right|<\varepsilon / 8 .
$$

We then require $\delta$ to be small enough so that

$$
|O(\varepsilon \delta)|<\varepsilon / 16,
$$

and then for sufficiently small $\varepsilon$ 's we will have

$$
\left|O\left(\varepsilon^{2} / \delta\right)\right|<\varepsilon / 16
$$


for $\varepsilon$ 's small enough. If $a=-\varepsilon / 2$ the right-hand-side of (8.38) will be negative, it will be positive if $a=\varepsilon / 2$, and continuity shows existence of an $a$ that leads to a solution of the full constraint equations.

Equation (8.34a) shows that $m$ will be close to $\sum_{i=0}^{2 N} m_{i}$, which gives the desired control of the ratio $\delta / m$ if all the $m_{i}$ 's are of the same sign. It follows that the previous construction applies in this case. Clearly the sign condition is not necessary, and there exist several other families of $m$ parameters which will give the desired control.

We can now repeat the whole previous construction by gluing boosted Kerr initial data centred on the $\vec{x}_{i}$ 's, with a small boost parameter, to the solution $(K, g)$ just obtained with the same remaining parameters. If $(K, g)$ has no KID's, then we will obtain a new smooth solution from Theorem 5.9 and Corollary 5.11 provided that the boost parameters are small enough. We note that the initial data $(K, g)$ will have no KID's except when all the $\vec{x}_{i}$ 's are aligned along, say, the $z$-axis, with all the $\vec{\omega}(i)$ 's pointing in the $\partial_{z}$ direction. One expects that a variation of the above arguments would still give existence of solutions, but we have not investigated this point any further.

The mass of the solutions obtained above, as seen from the end $r \geqslant R_{2}$, might be very small. One can now make a usual rescaling $m \rightarrow \lambda m, r \rightarrow \lambda r, g_{\vec{m}} \rightarrow \lambda^{-2} g_{\vec{m}}$, to obtain any value of the final mass $m$.

We emphasize that the mass parameters $m_{i}$ and $m_{0}$ are only restricted in absolute value, so solutions $\left(0, g_{\vec{M}}\right)$ with some of the $m_{i}$ 's negative or zero, and/or $m_{0}$ negative or zero, and $m$ negative, can be constructed. For instance, a zero value of $m_{i}$ will correspond to metrics which can be $C^{k}$ matched to a flat metric on $B\left(\vec{x}_{i}, r_{i}\right)$. One can actually also obtain $a=0$, or $m=0$, or both: it suffices to repeat the above argument with prescribed values $a m=0$ and $m_{i}, i=1, \ldots, 2 N$, adjusting $m_{0}$ and/or $a_{0}$ rather than $m$ and $a$. Arguing as before one can obtain a family of non-trivial vacuum initial data which are Minkowskian on an exterior region $\mathbb{R}^{3} \backslash B(0, R)$. (Clearly $m=0$ implies that at least one of the $m_{i}$ 's, $i \geqslant 0$, is negative, unless they all vanish.) 


\section{APPENDIX A}

\section{WEIGHTED SOBOLEV AND WEIGHTED HÖLDER SPACES}

Let $\phi$ and $\psi$ be two smooth strictly positive functions on $M$. For $k \in \mathbb{N}$ let $H_{\phi, \psi}^{k}(g)$ be the space of $H_{\mathrm{loc}}^{k}$ functions or tensor fields such that the norm ${ }^{(1)}$

$$
\|u\|_{H_{\phi, \psi}^{k}(g)}:=\left(\int_{M}\left(\sum_{i=0}^{k} \phi^{2 i}\left|\nabla^{(i)} u\right|_{g}^{2}\right) \psi^{2} d \mu_{g}\right)^{1 / 2}
$$

is finite, where $\nabla^{(i)}$ stands for the tensor

$$
\underbrace{\nabla \cdots \nabla}_{i \text { times }} u
$$

with $\nabla$ - the Levi-Civita covariant derivative of $g$; we assume throughout that the metric is at least $W_{\text {loc }}^{1, \infty}$; higher differentiability will be usually indicated whenever needed. For $k \in \mathbb{N}$ we denote by $\stackrel{\circ}{H}_{\phi, \psi}^{k}$ the closure in $H_{\phi, \psi}^{k}$ of the space of $H^{k}$ functions or tensors which are compactly (up to a negligible set) supported in $M$, with the norm induced from $H_{\phi, \psi}^{k}$. The $\stackrel{\circ}{H}_{\phi, \psi}^{k}$ 's are Hilbert spaces with the obvious scalar product associated to the norm (A.1). We will also use the following notation

$$
\stackrel{\circ}{H}^{k}:=\stackrel{\circ}{H}_{1,1}^{k}, \quad L_{\psi}^{2}:=\stackrel{\circ}{H}_{1, \psi}^{0}=H_{1, \psi}^{0},
$$

so that $L^{2} \equiv \stackrel{\circ}{H}^{0}:=\stackrel{\circ}{H}_{1,1}^{0}$. We set

$$
W_{\phi}^{k, \infty}:=\left\{u \in W_{\text {loc }}^{k, \infty} \text { such that } \phi^{i}\left|\nabla^{(i)} u\right|_{g} \in L^{\infty}\right\},
$$

with the obvious norm, and with $\nabla^{(i)} u$ - the distributional derivatives of $u$.

${ }^{(1)}$ The reader is referred to $[\mathbf{5 , 6 , 4 4}]$ for a discussion of Sobolev spaces on Riemannian manifolds. 
For $\phi$ and $\varphi$ - smooth strictly positive functions on $\mathrm{M}$, and for $k \in \mathbb{N}$ and $\alpha \in[0,1]$, we define $C_{\phi, \varphi}^{k, \alpha}$ the space of $C^{k, \alpha}$ functions or tensor fields for which the norm

$$
\begin{aligned}
\|u\|_{C_{\phi, \varphi}^{k, \alpha}(g)}=\sup _{x \in M} \sum_{i=0}^{k}\left(\left\|\varphi \phi^{i} \nabla^{(i)} u(x)\right\|_{g}\right. & \\
& \left.+\sup _{0 \neq d_{g}(x, y) \leqslant \phi(x) / 2} \varphi(x) \phi^{i+\alpha}(x) \frac{\left\|\nabla^{(i)} u(x)-\nabla^{(i)} u(y)\right\|_{g}}{d_{g}^{\alpha}(x, y)}\right)
\end{aligned}
$$

is finite.

We will only consider weight functions with the property that there exists $\ell \in$ $\mathbb{N} \cup\{\infty\}$ such that ${ }^{(2)}$ for $0 \leqslant i<\ell$ we have

$$
\left|\phi^{i-1} \nabla^{(i)} \phi\right|_{g} \leqslant C_{i}, \quad\left|\phi^{i} \psi^{-1} \nabla^{(i)} \psi\right|_{g} \leqslant C_{i},
$$

for some constants $C_{i}$. This implies that for $0 \leqslant i<\ell$ and for all $k \in \mathbb{N}$ it holds that

$$
\left|\phi^{i-k} \nabla^{(i)} \phi^{k}\right|_{g} \leqslant C_{i, k}, \quad\left|\phi^{i} \psi^{-k} \nabla^{(i)} \psi^{k}\right|_{g} \leqslant C_{i, k} .
$$

It follows that for $m, s \in \mathbb{N}$ and for $0 \leqslant i+k<\ell$ the maps

$$
\begin{gathered}
\psi^{-m} \phi^{i-s} \nabla^{(i)}\left(\phi^{s} \psi^{m} \cdot\right): \stackrel{\circ}{H}_{\phi, \psi}^{k+i} \longmapsto \stackrel{\circ}{H}_{\phi, \psi}^{k}, \\
\psi^{-m} \phi^{i-s} \nabla^{(i)}\left(\phi^{s} \psi^{m} \cdot\right): W_{\phi}^{k+i, \infty} \longmapsto W_{\phi}^{k, \infty}, \\
\psi^{-m} \phi^{-s} \nabla^{(i)}\left(\phi^{i+s} \psi^{m} \cdot\right): \stackrel{\stackrel{H}{H}}{\phi, \psi}^{k+i} \longmapsto \stackrel{\circ}{H}_{\phi, \psi}^{k}, \\
\psi^{-m} \phi^{-s} \nabla^{(i)}\left(\phi^{i+s} \psi^{m} \cdot\right): W_{\phi}^{k+i, \infty} \longmapsto W_{\phi}^{k, \infty},
\end{gathered}
$$

are continuous and bounded. If the function $\varphi$ satisfies the same condition (A.3) as $\psi$, then we can replace $\stackrel{\circ}{H}_{\phi, \psi}^{j}$ by $C_{\phi, \varphi}^{j, \alpha}$ in (A.4).

The following situations will be of main interest to us:

- If $\partial M$ is compact, smooth, and non-empty (see section 5), we will use for $\phi=x$ a function which is a defining function for the boundary, at least in a neighborhood of the boundary; that is, any smooth non-negative function on $\bar{M}$ such that $\partial M$ is precisely the zero-level set of $x$, with $d x$ without zeros on $\partial M$. Then $\psi$ will be a power of $x$ on a neighborhood of $\partial M$. Condition (A.2) will hold if $g$ has $\left(W_{x}^{\ell-1, \infty}, l-1\right)$ behavior at $\partial M$ in the sense of Definition 5.3.

- If $M$ contains an asymptotically flat region (see Section 7), $\phi$ will behave as $r$ and $\psi$ will behave as a power of $r$ in the asymptotically flat region; (A.2) will hold if $g$ is $W_{r}^{\ell-1, \infty}$-asymptotically flat.

- If $M$ contains a conformally compactifiable region (see Section 6), then in a neighborhood of the conformal boundary $\phi$ will be taken to be 1 , while $\psi$ will be a power of the defining function of the conformal boundary.

- Exponentially weighted versions of the above will also be considered.

${ }^{(2)}$ Conditions (A.2) will typically impose $\ell$ restrictions on the behavior of the metric and its derivatives in the asymptotic regions; it is therefore essential to allow $\ell<\infty$ if one does not wish to impose an infinite number of such conditions.

MÉMOIRES DE LA SMF 94 
In all those situations one can obtain elliptic estimates in weighted spaces for the equations considered here by covering and scaling arguments together with the standard interior elliptic estimates on compact balls $(c f .$, e.g. $[\mathbf{1}, \mathbf{3}, \mathbf{7}, \mathbf{1 6}, \mathbf{4 1}, \mathbf{5 2}])$. We will refer to this as the scaling property.

More precisely, we shall say that the scaling property holds (with respect to some weighted Sobolev spaces with weight functions $\psi$ and $\phi$, and/or weighted Hölder spaces with weight functions $\varphi$ and $\phi$, whichever ones are being used will always be obvious from the context) if there exists a covering of $M$ by a family of sets $\Omega_{\alpha}$, for $\alpha$ in some index set $I$, together with scaling transformations $\phi_{\alpha}: \Omega_{\alpha} \rightarrow \widehat{\Omega}_{\alpha}$ on each of the sets $\Omega_{\alpha}$, such that the transformed fields $\left(\widehat{K}_{\alpha}, \widehat{g}_{\alpha}\right)$ on $\Omega_{\alpha}$ are in ${ }^{(3)}$ in $W^{3, \infty}\left(\widehat{\Omega}_{\alpha}\right) \times$ $W^{4, \infty}\left(\widehat{\Omega}_{\alpha}\right)$, and such that the usual interior elliptic estimates on the $\widehat{\Omega}_{\alpha}$ 's can be pieced together to a weighted estimate, such as (3.21), for the original fields. Some sufficient conditions for the scaling property are discussed in Appendix B. We note that the scaling transformation of the fields on $\widehat{\Omega}_{\alpha},(K, g) \rightarrow\left(\widehat{K}_{\alpha}, \widehat{g}_{\alpha}\right)$, will typically consist of a pull-back of the fields, accompanied perhaps by a constant conformal rescaling. The "scaling property" is a condition both on the metric $g$, the extrinsic curvature tensor $K$, and on the weight functions involved: indeed, both the metric coefficients, the connection coefficients, as well as their derivatives, etc., which appear in our equations must have appropriate behavior under the above transformations so that the required estimates can be established.

${ }^{(3)}$ It is conceivable that in some situations less a priori regularity on the $\left(\widehat{K}_{\alpha}, \widehat{g}_{\alpha}\right)$ 's can be assumed, but this is the setup which seems to play the most important role in our paper; the reader should be able to adapt the differentiability conditions to his needs if required. 



\section{APPENDIX B}

\section{SUFFICIENT CONDITIONS FOR THE SCALING PROPERTY}

In this section we present some sufficient conditions on the functions $\phi$ and $\varphi$ which guarantee that the spaces $C_{\phi, \varphi}^{k, \alpha}(g)$ satisfies the scaling property. We give some examples of such spaces. We assume that the manifold $M$ is an open subset of $\mathbb{R}^{n}$, and that the elliptic operator we work with is an operator on functions. The result generalizes to tensor fields on manifolds by using coordinate patches, together with covering arguments.

We assume that $\phi$ and $\varphi$ verify (A.2). For all $p \in M$, we denote by $B_{p}$, the open ball of centre $p$ with radius $\phi(p) / 2$. We require that ${ }^{(1)}$ for all $p \in M$,

$$
B(p, \phi(p)) \subset M \text {. }
$$

For $p \in M$, we define

$$
\varphi_{p}: B(0,1 / 2) \ni z \longmapsto p+\phi(p) z \in B_{p} .
$$

For all functions $u$ on $M$ and all multi-indices $\gamma$ we have

$$
\partial_{z}^{\gamma}\left(u \circ \varphi_{p}\right)=\phi(p)^{|\gamma|}\left(\partial^{\gamma} u\right) \circ \varphi_{p}
$$

Let $P(p, \partial)$ be a strictly elliptic (e.g., in the sense of Agmon-Douglis-Nirenberg) operator of order $m$ on $M$ and set

$$
\left(P_{\phi} u\right)(p):=[P(\cdot, \phi \partial) u](p),
$$

note that in our context $P_{\phi}$ will be elliptic uniformly degenerate whenever $\phi(p)$ approaches zero in some regions. We assume that the coefficients of $P$ are in $C_{\phi, 1}^{k, \alpha}(M)$. For all $p \in M$, we define the elliptic operator $Q_{p}$ on $B(0,1 / 2)$ by

$$
Q_{p}(z, \partial):=P\left(\varphi_{p}(z),(\phi(p))^{-1} \phi \circ \varphi_{p}(z) \partial\right),
$$

\footnotetext{
(1) It suffices to assume that there exists $\mu>0$ such that for all $p \in M, B(p, \mu \phi(p)) \subset M$, as changing $\phi$ to $\mu \phi$ for a positive constant $\mu$ leads to equivalent norms. This is actually the condition needed in the asymptotically flat case, as (B.1) will typically not be satisfied there. For convenience we assume in (B.1) that any such rescalings have already been done.
} 
we then have

$$
Q_{p}\left(u \circ \varphi_{p}\right)=\left(P_{\phi} u\right) \circ \varphi_{p}
$$

We assume that there exist a constant $C_{1}>0$ such that for all $p \in M$ and all $y \in B_{p}$, we have

$$
C_{1}^{-1} \phi(p) \leqslant \phi(y) \leqslant C_{1} \phi(p) .
$$

Then the $C^{k, \alpha}(B(0,1 / 2))$ norms of the coefficients of $Q_{p}$ are bounded by the $C_{\phi, 1}^{k, \alpha}(M)$ norm of the coefficients of $P$. On the other hand, $Q_{p}$ is strictly elliptic and, by the usual interior elliptic estimates, there exists $C>0$ which does not depend on $p$ and $v$ such that for all functions $v \in L^{2}(B(0,1 / 2))$, for which $Q_{p} v$ is in $C^{k, \alpha}(B(0,1 / 2))$, we have $v \in C^{k+m, \alpha}(B(0,1 / 4))$ and

$$
\|v\|_{C^{k+m, \alpha}(B(0,1 / 4))} \leqslant C\left(\left\|Q_{p} v\right\|_{C^{k, \alpha}(B(0,1 / 2))}+\|v\|_{L^{2}(B(0,1 / 2))}\right) .
$$

So if $u$ is in $L_{\varphi \phi^{-n / 2}}^{2}(M)$ with $P u \in C_{\phi, \varphi}^{k, \alpha}(M)$, then $u \in C_{l o c}^{k+m, \alpha}$. Now, we assume that there exists a constant $C_{2}>0$ such that for all $p \in M$ and all $y \in B_{p}$, we have

$$
C_{2}^{-1} \varphi(p) \leqslant \varphi(y) \leqslant C_{2} \varphi(p) .
$$

For $p \in M$, we define $B_{p}^{\prime}$ the ball of centre $p$ and radius $(1 / 4) \phi(p)$. It follows from (B.2) that there is a $p$-independent number $N$ such that each $B_{p}$ is covered by $N$ balls $B_{p_{i}(p)}^{\prime}, i=1, \ldots, N$. We then have (the second and the last inequalities come from (B.3))

$$
\begin{aligned}
\|u\|_{C_{\phi, \varphi}^{k, \alpha}(M)} \leqslant C \sup _{p \in M}\|u\|_{C_{\phi, \varphi}^{k+m, \alpha}\left(B_{p}^{\prime}\right)} \\
\leqslant C \sup _{p \in M}\left(\varphi(p)\|u\|_{C_{\phi(p), 1}^{k+m, \alpha}\left(B_{p}^{\prime}\right)}\right) \\
\leqslant C \sup _{p \in M}\left(\varphi(p)\left\|u \circ \varphi_{p}\right\|_{C^{k+m, \alpha}\left(\varphi_{p}^{-1}\left(B_{p}^{\prime}\right)\right)}\right) \\
=C \sup _{p \in M}\left(\varphi(p)\left\|u \circ \varphi_{p}\right\|_{C^{k+m, \alpha}(B(0,1 / 4))}\right) \\
\leqslant C \sup _{p \in M}\left[\varphi(p)\left(\left\|P_{\phi} u \circ \varphi_{p}\right\|_{C^{k, \alpha}(B(0,1 / 2))}+\left\|u \circ \varphi_{p}\right\|_{L^{2}(B(0,1 / 2))}\right)\right] \\
\leqslant C\left[\sup _{p \in M}\left(\varphi(p)\|P u\|_{C_{\phi(p), 1}^{k, \alpha}\left(B_{p}\right)}\right)+\sup _{p \in M}\left(\|u\|_{L_{\varphi \phi^{-n / 2}}^{2}\left(B_{p}\right)}\right)\right] \\
\leqslant C\left(\|P u\|_{C_{\phi, \varphi}^{k, \alpha}(M)}+\|u\|_{L_{\varphi^{2}}^{2}-n / 2}(M)\right) .
\end{aligned}
$$

In particular $u \in C_{\phi, \varphi}^{k+m, \alpha}(M)$. An identical calculation gives

$$
\|u\|_{C_{\phi, \varphi}^{k+m, \alpha}(M)} \leqslant C\left(\|P u\|_{C_{\phi, \varphi}^{k, \alpha}(M)}+\|u\|_{L_{\varphi}^{\infty}(M)}\right) .
$$

A similar scaling calculation, together with a summation over a set of $B_{p_{i}}$ 's forming an appropriate covering of $M$, gives the corresponding inequality in weighted Sobolev space. We thus obtain:

Lemma B.1. - If $\phi$ and $\varphi$ satisfy the condition (A.2) with $\psi$ replaced by $\varphi$, together with (B.1), (B.2) and (B.3), then the spaces $C_{\phi, \varphi}^{k, \alpha}$ verify the scaling property. 
As already mentioned, near a compact boundary a standard example of functions satisfying the above requirement is $\phi=x$, and $\varphi-$ a power of $x$, with $x-$ a defining function for the boundary. Another example is $\varphi=e^{-s / x}$, where $s \in \mathbb{R}$, and $\phi=x^{2}$. In fact in that context, $x$ is equivalent to $d(\cdot, \partial M)$. For sufficiently regular metrics (e.g., $g \in C^{\ell}$ ) we have (A.2), while the choice of $\phi$ guarantees (B.1). For (B.2) we compute for all $q \in B_{p}$, by the triangle inequality,

$$
d(p, \partial M)-d(p, q)<d(q, \partial M) \leqslant d(p, \partial M)+d(p, q) .
$$

Then, since $d(p, q)<x(p)^{2} / 2$ for $q \in B_{p}$,

$$
d(p, \partial M)-x(p)^{2} / 2<d(q, \partial M) \leqslant d(p, \partial M)+x(p)^{2} / 2 .
$$

From (B.1) we have that $x(p)^{2}<d(p, \partial M)$, giving

$$
d(p, \partial M) / 2<d(q, \partial M) \leqslant 3 d(p, \partial M) / 2,
$$

and as $x$ is equivalent to $d(., \partial M)$ we obtain (B.2). Now for all $q \in B_{p}$,

$$
e^{-s / x(p)} e^{s / x(q)}=e^{-s(x(p)-x(q)) / x(p) x(q)},
$$

but $|x(p)-x(q)|$ is bounded by some constant times $x(p)^{2}$ and $x(p) x(q)$ is equivalent to $x(p)^{2}$ so we obtain (B.3).

We note that if $\varphi_{1}$ and $\varphi_{2}$ satisfy (B.3), then $\varphi_{1} \varphi_{2}$ also will. It follows that $\varphi=x^{\alpha} e^{s / x}$ can also be used as a weighting function in our context with $\phi=x^{2}$ for all $\alpha, s \in \mathbb{R}$.

In asymptotically flat regions the standard choice is $\varphi=r^{\alpha}$, for some $\alpha \in \mathbb{R}$, and $\phi=r$. Another one is $\phi=1$ and $\varphi=e^{s r}$, where $s \in \mathbb{R}$; in that case (B.1), (B.2) and (B.3) are evident. 



\section{APPENDIX C}

\section{WEIGHTED POINCARÉ INEQUALITIES}

We start with some general inequalities on an open manifold $M$, then we will apply them to all the cases of interest to us. All the integrals are always calculated with respect to the natural Riemannian measure $d \mu=d \mu_{g}=d \mu(g)$ with respect to the metric at hand, in local coordinates $d \mu=\sqrt{\operatorname{det} g_{i j}} d^{n} x$.

We start with a lemma:

LEMMA C.1. - Let $u$ be a $C^{1}$ compactly supported tensor field on $M$, and let $w$ be a $C^{2}$ function defined in a neighborhood of the support of $u$, then

$$
\int_{M}|\nabla u|^{2} \geqslant \int_{M}\left(-|\nabla w|^{2}+\Delta w\right)|u|^{2} .
$$

Proof. - $\quad \int_{M}|\nabla u|^{2}+|\nabla w|^{2}|u|^{2}+2 u \nabla_{i} u \nabla^{i} w=\int_{M}|\nabla u+(\nabla w) u|^{2} \geqslant 0$. (By an abuse of notation, here and below we write $u \nabla_{i} u \nabla^{i} w$ for $h\left(u, \nabla_{\nabla w} u\right)$, where $h$ is the metric, constructed using $g$, on the tensor bundle relevant to the tensor character of $u$.) An integration by parts leads to

$$
\int_{M}|\nabla u|^{2}+|\nabla w|^{2}|u|^{2}-\Delta w|u|^{2} \geqslant 0,
$$

so that

$$
\int_{M}|\nabla u|^{2} \geqslant \int_{M}-|\nabla w|^{2}|u|^{2}+\Delta w|u|^{2}
$$

Proposition C.2. - Let $u$ be a $C^{1}$ compactly supported tensor field on $M$, and let $w, v$ be two $C^{2}$ functions defined on a neighborhood of the support of $u$, then

$$
\int_{M} e^{2 v}|\nabla u|^{2} \geqslant \int_{M} e^{2 v}\left[\Delta v+\Delta w+|\nabla v|^{2}-|\nabla w|^{2}\right]|u|^{2} .
$$


Proof. - Returning to the proof of Lemma C.1, with $u$ replaced by $e^{v} u$ gives

$$
\begin{aligned}
\int_{M} e^{2 v}\left[|\nabla u|^{2}+|\nabla v|^{2}|u|^{2}+2 u \nabla_{i} u \nabla^{i} v\right] & =\int_{M} e^{2 v}|\nabla u+u \nabla v|^{2} \\
& \geqslant \int_{M} e^{2 v}\left(-|\nabla w|^{2}+\Delta w\right)|u|^{2} .
\end{aligned}
$$

An integration by parts transforms the left-hand-side of the first line of Equation (C.2) into

$$
\int_{M} e^{2 v}\left\{\left[|\nabla u|^{2}+|\nabla v|^{2}|u|^{2}\right]-\Delta v|u|^{2}-2|\nabla v|^{2}|u|^{2}\right\}
$$

so that (C.2) can be rewritten as

$$
\int_{M} e^{2 v}|\nabla u|^{2} \geqslant \int_{M} e^{2 v}\left[\Delta v+\Delta w+|\nabla v|^{2}-|\nabla w|^{2}\right]|u|^{2} .
$$

\section{C.1. Application: compact boundaries}

Let $x$ be any twice-differentiable defining function for $\partial M$. We shall consider metrics $g$ which are in $W_{\text {loc }}^{1, \infty}$ on $M$ and continuous on $\bar{M}$. We shall further suppose that the Hessian Hess $x:=\nabla \nabla x$ of $x$ satisfies

$$
|\operatorname{Hess} x|=o\left(x^{-1}\right) .
$$

Equation (C.3) will obviously hold if $g$ is smooth on $\bar{M}$; it is, however, natural to consider metrics of lower differentiability class when $\partial M$ corresponds to a conformal boundary at infinity. (Actually, in this section it would suffice to assume that $\Delta x=o\left(x^{-1}\right)$; however, the stronger hypothesis (C.3) will be required in our further considerations.)

We will work in a neighborhood of $\partial M$ small enough so that $|d x|$ is bounded away from zero there. The following result is well known (compare [63, Lemma 1, Section 3.2.6]), we give a proof since we need to control the constant in Equation (C.4) below; the calculation can be traced back to those in [52]:

Proposition C.3. - For any $\varepsilon>0$ and $s \neq-1 / 2$ there exists $x_{\varepsilon, s}>0$ such that for any differentiable tensor field $u$ with compact support in $\left\{0<x<x_{\varepsilon, s}\right\}$ we have

$$
\int_{M} x^{2 s+2}|\nabla u|^{2} d \mu \geqslant\left\{(s+1 / 2)^{2}-\varepsilon\right\} \int_{M} x^{2 s}|u|^{2}|d x|^{2} d \mu .
$$

Proof. - We use Proposition C.2, choosing $v=(s+1) \ln (x)$ one has $d v=(s+1) d x / x$ and

$$
\Delta v=-(s+1)|d x|^{2} / x^{2}+(s+1) \Delta x / x=-(s+1+o(1))|d x|^{2} / x^{2} .
$$

It follows that

$$
|d v|^{2}+\Delta v=\left((s+1)^{2}-s-1+o(1)\right)|d x|^{2} / x^{2} .
$$

MÉMOIRES DE LA SMF 94 
Choosing $w=-\frac{1}{2} \ln (x)$ we have that

$$
-|d w|^{2}+\Delta w=(1 / 4+o(1))|d x|^{2} / x^{2} .
$$

Proposition C.4. - For any $\varepsilon>0, t, s \in \mathbb{R}$ there exists $x_{\varepsilon, s, t}>0$ such that for any differentiable tensor field $u$ with compact support in $\left\{0<x<x_{\varepsilon, s, t}\right\}$ we have

$$
\int_{M} e^{-2 s / x} x^{2 t}|\nabla u|^{2} d \mu \geqslant\left\{s^{2}-\varepsilon\right\} \int_{M} e^{-2 s / x}|u|^{2} x^{2 t-4}|d x|^{2} d \mu .
$$

Proof. - We again use Proposition C.2 with $v=-s / x+t \ln x$ and $w=0$, one then has $d v=s d x / x^{2}+t d x / x$ and

$$
\Delta v=-2 s|d x|^{2} / x^{3}+s \Delta x / x^{2}-t|d x|^{2} / x^{2}+t(\Delta x) / x=o(1)|d x|^{2} / x^{4} .
$$

It follows that

$$
|d v|^{2}+\Delta v=\left(s^{2}+o(1)\right)|d x|^{2} / x^{4} .
$$

then we obtain (C.6).

\section{C.2. Application: asymptotically flat metrics}

We shall also need a weighted Poincaré inequality for metrics $g$ defined on $\mathbb{R}^{n} \backslash\{r \geqslant R\}$ for some $R$, satisfying the following requirement: for every $\varepsilon>0$ there exists $R_{\varepsilon}<\infty$ such that

$$
\left|g_{i j}-\delta_{i j}\right| \leqslant \varepsilon \text { on }\left\{r \geqslant R_{\varepsilon}\right\}
$$

We shall also require that

$$
\Delta r-(n-1)|\nabla r|^{2} / r=o(1)
$$

(recall that the right-hand-side above is zero for a flat metric). One then has the following $[\mathbf{7}, \mathbf{2 1}]$; we give a proof for completeness ${ }^{(1)}$ :

Proposition C.5. - Suppose that (C.7)-(C.8) hold. Then for any $s \in \mathbb{R}$ and $\varepsilon>0$ there exists $R_{s, \varepsilon}<\infty$ such that for any $C^{1}$ tensor field $u$ with compact support included in $\left\{r>R_{s, \varepsilon}\right\}$ it holds that

$$
\int r^{-2 s-n+2}|\nabla u|^{2} d \mu \geqslant\left(s^{2}-\varepsilon\right) \int r^{-2 s-n}|u|^{2} d \mu .
$$

Proof. - We use Proposition C.2 with $v=(-s+1-n / 2) \ln r$ and $w=[(n-2) \ln r] / 2$. We just recall that when $f=c \ln r$, we have

$$
\nabla f=c \nabla r / r
$$

and

$$
\Delta f=c \Delta r / r-c|\nabla r|^{2} / r^{2}=c(n-2)|\nabla r|^{2} / r^{2}+o(1 / r) .
$$

(1) Actually the case $n=2$ does not seem to have appeared in the published literature so far. 
So

$$
\Delta v+|\nabla v|^{2}=\left(s^{2}-(n-2)^{2} / 4\right)|\nabla r|^{2} / r^{2}+o(1 / r),
$$

and

$$
\Delta w-|\nabla w|^{2}=\left((n-2)^{2} / 4\right)|\nabla r|^{2} / r^{2}+o(1 / r) .
$$

Proposition C.6. - Suppose that (C.7)-(C.8) hold. Then for any $\varepsilon>0$ there exists $R_{s, \varepsilon}<\infty$ such that for any $C^{1}$ tensor field $u$ with compact support included in $\{r>$ $\left.R_{s, \varepsilon}\right\}$ it holds that

$$
\int e^{-2 s r}|\nabla u|^{2} d \mu \geqslant\left(s^{2}-\varepsilon\right) \int e^{-2 s r}|\nabla r|^{2}|u|^{2} d \mu .
$$

Proof. - We use Proposition C.2 with $v=-s r$ and $w=0$. Then $\nabla v=-s \nabla r$ and $\Delta v=-s \Delta r=-s(n-1)|\nabla r|^{2} / r+o(1)=o(1)|\nabla r|^{2}$. So

$$
\Delta v+|\nabla v|^{2}=\left(s^{2}+o(1)\right)|\nabla r|^{2} .
$$

\section{C.3. Application: conformally compact manifolds}

Here, as elsewhere, $n$ denotes the dimension of $M$. We recall that we have $g=x^{-2} \bar{g}$ then

$$
(\Gamma-\bar{\Gamma})_{i j}^{k}=-x^{-1}\left(2 \delta_{(i}^{k} \bar{\nabla}_{j)} x-\bar{g}_{i j} \bar{\nabla}^{k} x\right) .
$$

In particular, we have

$$
\nabla_{i} \nabla_{j} x=\bar{\nabla}_{i} \bar{\nabla}_{j} x+x^{-1}\left(2 \bar{\nabla}_{i} x \bar{\nabla}_{j} x-\bar{g}_{i j}|d x|_{\bar{g}}^{2}\right) .
$$

Throughout this section we use the symbol $|\cdot|$ for $|\cdot|_{g}$, but we write explicitly $|\cdot|_{\bar{g}}$ when the $\bar{g}$ metric is involved.

Proposition C.7. - For any $\varepsilon>0$ and $s \in \mathbb{R}$, there exists $x_{\varepsilon, s}>0$ such that for any differentiable tensor field $u$ with compact support in $\left\{0<x<x_{\varepsilon, s}\right\}$ we have

$$
\int_{M} x^{2 s}\left|x^{-2} \nabla(x u)\right|^{2} d \mu \geqslant\left\{[s-(n+3) / 2]^{2}-\varepsilon\right\} \int_{M} x^{2 s-2}|u|^{2}|d x| \frac{2}{g} d \mu .
$$

Proof. - We use Proposition C.2, choosing $v=(s-2) \ln x$ one has $d v=(s-2) d x / x$ and

$$
\Delta v=(s-2)\left[-|d x|^{2} / x^{2}+\Delta x / x\right]=(s-2)(1-n)|d x|^{2} / x^{2}+o(1) .
$$

It follows that

$$
|d v|^{2}+\Delta v=\left[(s-2)^{2}+(s-2)(1-n)+o(1)\right]|d x|^{2} / x^{2} .
$$

Choosing $w=[(1-n) \ln x] / 2$ we have that

$$
-|d w|^{2}+\Delta w=\left[-(1-n)^{2} / 4+(1-n)^{2} / 2+o(1)\right]|d x|^{2} / x^{2} .
$$




\section{APPENDIX D}

\section{WEIGHTED ESTIMATES FOR VECTOR FIELDS}

In this section we give some estimates for the operator $S$ which associates to a vector field $Y$ one half of the Lie derivative of the metric along $Y$ :

$$
S(Y)_{i j}=\frac{1}{2}\left(\mathcal{L}_{Y} g\right)_{i j}:=\frac{1}{2}\left(\nabla_{i} Y_{j}+\nabla_{j} Y_{i}\right)
$$

As it will be often used, we recall that

$$
\operatorname{tr}(S(Y))=\operatorname{div} Y=\nabla^{i} Y_{i}
$$

Lemma D.1. - For all vector fields $V$ and all vector fields $Y$ with compact support we have the equality

$$
\int_{M}\left[S(Y)+\frac{1}{2} \operatorname{tr}(S(Y)) g\right](Y, V)=-\frac{1}{2} \int_{M} \nabla V(Y, Y)+\frac{1}{2} \operatorname{div}(V)|Y|^{2} .
$$

Proof. - We integrate by parts the two terms on the right-hand side of the equality

$$
S(Y)_{i j} Y^{i} V^{j}=\frac{1}{2}\left(\nabla_{i} Y_{j} Y^{i} V^{j}+\nabla_{j} Y_{i} Y^{i} V^{j}\right) .
$$

Proposition D.2. - For all functions $u$, all vector fields $V$ and all vector fields $Y$ with compact support we have the equality

$$
\begin{aligned}
& \int_{M} e^{2 u} {\left[S(Y)+\frac{1}{2} \operatorname{tr}(S(Y)) g\right](Y, V) } \\
&=-\frac{1}{2} \int_{M} e^{2 u}\left\{\nabla V(Y, Y)+\frac{1}{2} \operatorname{div}(V)|Y|^{2}+\langle d u, V\rangle|Y|^{2}+2\langle d u, Y\rangle\langle V, Y\rangle\right\} .
\end{aligned}
$$


Proof. - We use Lemma D.1 with $Y$ replaced by $e^{u} Y$, so that

$$
\begin{aligned}
& \int_{M}\left[S\left(e^{u} Y\right)+\frac{1}{2} \operatorname{tr}\left(S\left(e^{u} Y\right)\right) g\right]\left(e^{u} Y, V\right) \\
& =\int_{M} e^{2 u}\left\{\left[S(Y)+\frac{1}{2} \operatorname{tr}(S(Y)) g\right](Y, V)+\frac{1}{2}\left(\nabla_{i} u Y_{j}+\nabla_{j} u Y_{i}+\langle d u, Y\rangle g_{i j}\right) Y^{i} V^{j}\right\} \\
& =\int_{M} e^{2 u}\left\{\left[S(Y)+\frac{1}{2} \operatorname{tr}(S(Y)) g\right](Y, V)+\frac{1}{2}\langle d u, V\rangle|Y|^{2}+\langle d u, Y\rangle\langle V, Y\rangle\right\} .
\end{aligned}
$$

Proposition D.3. - For all vector fields $Y$ with compact support and functions $u$ and $v$ supported in a neighborhood of the support of $Y$, we have

$$
\begin{aligned}
&-2 \int_{M} v e^{2 u} S(Y)(\nabla v, \nabla v)\langle d v, Y\rangle \\
& \quad=\int_{M} e^{2 u}\langle d v, Y\rangle\left[\langle d v, Y\rangle\left(|d v|^{2}+v \Delta v+2 v\langle d v, d u\rangle\right)+2 v \nabla \nabla v(Y, \nabla v)\right] .
\end{aligned}
$$

Proof. - Integrating $\nabla_{j}\left[(\langle d v, Y\rangle)^{2} v e^{2 u} \nabla^{j} v\right]$ over $M$ one has

$$
\begin{aligned}
-\int_{M}\langle d v, Y\rangle^{2}|d v|^{2} e^{2 u}=\int_{M}\{2\langle d v, Y\rangle[ & \nabla v(Y, \nabla v)+\nabla Y(\nabla v, \nabla v)] v e^{2 u} \\
& \left.+\langle d v, Y\rangle^{2}\left[v \Delta v e^{2 u}+2 v\langle d v, d u\rangle e^{2 u}\right]\right\},
\end{aligned}
$$

and $\nabla Y(\nabla v, \nabla v)=S(Y)(\nabla v, \nabla v)$.

\section{D.1. Application: compact boundaries}

We use here the notations of Section 5. Similarly to Section C.1 we assume that (C.3) holds.

Corollary D.4. - For all $s \in \mathbb{R}$ and all $\varepsilon>0$ there exists $x_{s, \varepsilon}>0$ such that for all vector fields $Y$ with compact support in $\left\{0<x<x_{s, \varepsilon}\right\}$ we have

$$
\begin{aligned}
\int_{M} x^{2 s}\left[S(Y)+\frac{1}{2} \operatorname{tr}\right. & (S(Y)) g](Y, \nabla x / x) \\
& =\frac{1}{2} \int_{M} x^{2 s-2}\left[\left(\frac{1}{2}-s\right)\left(|d x|^{2}|Y|^{2}+2\langle Y, \nabla x\rangle^{2}\right)+o(1)|Y|^{2}\right]
\end{aligned}
$$

Proof. - We apply Proposition D.2 with the vector field $V=\nabla x / x$ and the function $u=s \ln (x)$, so that $d u=s d x / x$ and

$$
\nabla \nabla u=-s \nabla x \nabla x / x^{2}+s \nabla \nabla x / x=-s \nabla x \nabla x / x^{2}+o\left(x^{-2}\right) .
$$


Corollary D.5. - For all $s, t \in \mathbb{R}$ and all $\varepsilon>0$ there exists $x_{s, t, \varepsilon}>0$ such that for all vector fields $Y$ with compact support in $\left\{0<x<x_{s, t, \varepsilon}\right\}$ we have

$$
\begin{aligned}
\int_{M} x^{2 t} e^{-2 s / x}[S(Y) & \left.+\frac{1}{2} \operatorname{tr}(S(Y)) g\right](Y, \nabla x / x) \\
= & \left.-\int_{M} x^{2 t-4} e^{-2 s / x}\left[\frac{s}{2}\left(|d x|^{2}|Y|^{2}+2\langle Y, \nabla x\rangle^{2}\right)+o(1)|Y|^{2}\right)\right] .
\end{aligned}
$$

Proof. - We apply Proposition D.2 with the vector field $V=\nabla x / x^{2}$ and the function $u=-s / x+t \ln x$, so that we have $d u=-s d x / x^{2}+t d x / x$ and $\nabla V=o\left(x^{-4}\right)$.

\section{D.2. Application: asymptotically flat metrics}

In this section we assume that (C.7) holds, while (C.8) will be strengthened to

$$
r \nabla \nabla r=\delta-\nabla r \nabla r+o(1 / r) .
$$

Corollary D.6. - For all $s \in \mathbb{R}$ and all vector fields $Y$ with compact support near infinity we have

$$
\begin{aligned}
\int_{M} r^{-2 s-n+2}\left[S(Y)+\frac{1}{2} \operatorname{tr}(S(Y)) g\right](Y, \nabla r / r) \\
=\frac{1}{2} \int_{M} r^{-2 s-n}\left[(s-1)|Y|^{2}+(2 s+n)\langle Y, \nabla r\rangle^{2}+o(1)|Y|^{2}\right] .
\end{aligned}
$$

Proof. - We apply Proposition D.2 with the vector field $V=\nabla r / r=\nabla(\ln (r))$ and the function $u=(-s-n / 2+1) \ln (r)$, then $d u=(-s-n / 2+1) \nabla r / r$ and

$$
\begin{aligned}
\nabla \nabla u & =(-s-n / 2+1)\left(-\nabla r \nabla r / r^{2}+\nabla \nabla r / r\right) \\
& =(-s-n / 2+1)\left(-2 \nabla r \nabla r / r^{2}+\delta / r^{2}\right)+o\left(r^{-2}\right),
\end{aligned}
$$

recall that $\lim _{r \rightarrow \infty}|\nabla r|^{2}=1$.

CoROLlaRY D.7. - For all $s \in \mathbb{R}$ and all vector fields $Y$ with compact support near infinity we have

$$
\begin{aligned}
\int_{M} e^{-2 s r}\left[S(Y)+\frac{1}{2} \operatorname{tr}(S(Y)) g\right] & (Y, \nabla r) \\
& =-\int_{M} e^{-2 s r}\left[s\left(|Y|^{2}+2\langle Y, \nabla r\rangle^{2}\right)+o(1)|Y|^{2}\right] .
\end{aligned}
$$

Proof. - We apply the Proposition D.2 with the vector field $V=\nabla r$ and the function $u=-s r$.

Corollary D.8. - For all vector fields $Y$ with compact support, we have

$$
\int_{M} r^{-2 s-n+1} S(Y)(\nabla r, \nabla r)\langle d r, Y\rangle=\int_{M} r^{-2 s-n}(s+o(1))\langle d r, Y\rangle^{2} .
$$


Proof. - We use Proposition D.3 with $v=r$ and $u=(-s-n / 2) \ln r$, together with the fact that

$$
r \nabla \nabla r=-\nabla r \nabla r+\delta+o(1)
$$

and $|d r|^{2}=1+o(1)$.

We obtain finally the desired inequalities:

Proposition D.9. - For all $s \neq 0,1$, there exist $C_{s}>0$ and $R(s)$ such that for all vector fields $Y$ with compact support in $\{r>R(s)\}$, we have

$$
\int_{M} r^{-2 s-n+2}|S(Y)|^{2} \geqslant C_{s} \int_{M} r^{-2 s-n}|Y|^{2} .
$$

Proof. - From Corollary D.8, for all $b>0$, we have

$\frac{b}{2} \int_{M} r^{-2 s-n+2}|S(Y)|^{2}+\frac{1}{2 b} \int_{M} r^{-2 s-n}|\nabla r|^{2}\langle d r, Y\rangle^{2} \geqslant \int_{M} r^{-2 s-n}(|s|+o(1))\langle d r, Y\rangle^{2}$.

We conclude by using Corollary D.6 and the inequality

$$
\left|\left[S(Y)+\frac{1}{2} \operatorname{tr}(S(Y)) g\right](Y, \nabla r / r)\right| \leqslant \frac{a}{2}\left|S(Y)+\frac{1}{2} \operatorname{tr}(S(Y)) g\right|^{2}+\frac{1}{2 a} r^{-2}|Y|^{2}|d r|^{2},
$$

for all $a>0$, together with the inequality

$$
|S(Y)|^{2} \geqslant \frac{1}{n}|\operatorname{tr} S(Y)|^{2} .
$$

\section{D.3. Application: conformally compact manifolds}

We recall that we have $g=x^{-2} \bar{g}$. Equation (C.12) gives

$$
\begin{aligned}
\nabla_{i} \nabla_{j}\left(x^{-1}\right) & =2 x^{-3} \nabla_{i} x \nabla_{j} x-x^{-2} \nabla_{i} \nabla_{j} x=x^{-3} \bar{g}_{i j}|d x|_{g}^{2}-x^{-2} \bar{\nabla}_{i} \bar{\nabla}_{j} x \\
& =x^{-1}|d x| \frac{2}{g} g_{i j}+\text { l.o. }
\end{aligned}
$$

where "l.o." denotes terms which are small compared to the remaining ones.

Corollary D.10. - For all $s \in \mathbb{R}$ and all vector fields $Y$ with compact support near the boundary we have

$$
\begin{aligned}
\int_{M} & x^{2 s}\left[S(Y)+\frac{1}{2} \operatorname{tr}(S(Y)) g\right](Y, \nabla x / x) \\
\quad= & \left.\frac{1}{2} \int_{M} x^{2 s}\left(\left(\frac{1+n}{2}-s\right)|d x| \frac{2}{g}\right)|Y|^{2}-(2 s+1)\langle d x / x, Y\rangle^{2}|d x|_{g}^{2}+o(1)|Y|^{2}\right) .
\end{aligned}
$$

Proof. - We apply Proposition D.2 with the vector field $V=\nabla\left(x^{-1}\right)=-x^{-2} \nabla x$ and the function $u=(s+1 / 2) \ln (x)$, using (D.4) one then has

$$
\nabla V=\nabla \nabla\left(x^{-1}\right)=x^{-1}|d x| \frac{2}{g} g+o\left(x^{-3}\right) .
$$

MÉMOIRES DE LA SMF 94 
Corollary D.11. — For all vector fields $Y$ with compact support, we have

$$
2 \int_{M} x^{2 s} S(Y)(\nabla x / x, \nabla x / x)\langle d x / x, Y\rangle=\int_{M} x^{2 s}(n-2 s-1+o(1))\langle d x / x, Y\rangle^{2}|d x| \frac{2}{g} .
$$

Proof. - We apply Proposition D.3 with $v=x^{-1}, u=(s+2) \ln x$, making use of (D.4).

Proposition D.12. - For all $s \neq(n+1) / 2,(n-1) / 2$ there exist constants $C_{s}>$ $0, x(s)>0$ such that for all differentiable vector fields $Y$ with compact support in $\{x<x(s)\}$ we have

$$
\int_{M} x^{2 s}|S(Y)|^{2} d \mu_{g} \geqslant C_{s} \int_{M} x^{2 s}|Y|^{2} d \mu_{g}
$$

Proof. — From Corollary D.11, for all $b>0$, we have

$$
\begin{aligned}
\frac{b}{2} \int_{M} x^{2 s}|S(Y)|^{2}+\frac{1}{2 b} \int_{M} x^{2 s} \mid \nabla x / & \left.x\right|^{2}\langle d x / x, Y\rangle^{2} \\
& \geqslant \int_{M} x^{2 s}(|n-1-2 s|+o(1))\langle d x / x, Y\rangle^{2}|d x|_{\frac{2}{g}}^{2}
\end{aligned}
$$

We conclude by using Corollary D.10 and the inequality

$$
\left|\left[S(Y)+\frac{1}{2} \operatorname{tr}(S(Y)) g\right](Y, \nabla x / x)\right| \leqslant \frac{a}{2}\left|S(Y)+\frac{1}{2} \operatorname{tr}(S(Y)) g\right|^{2}+\frac{1}{2 a}|Y|^{2}|d x / x|^{2},
$$

for all $a>0$, together with

$$
|S(Y)|^{2} \geqslant \frac{1}{n}|\operatorname{tr} S(Y)|^{2}
$$

From the last result we also get an inequality governing the Hessian operator:

Proposition D.13. - For all $s \neq(n+1) / 2,(n-1) / 2,(n-3) / 2$ there exist constants $C_{s}>0$ and $x(s)$ such that for all differentiable functions $N$ with compact support in $\{x<x(s)\}$, we have

$$
\int_{M} x^{2 s}|\nabla \nabla N-\Delta N g-N \operatorname{Ric} g|^{2} d \mu_{g} \geqslant C_{s} \int_{M} x^{2 s}\left(|N|^{2}+|\nabla N|^{2}\right) d \mu_{g} .
$$

Proof. — We will use Proposition D.12 with

$$
Y=x^{-1} \nabla N-N \nabla\left(x^{-1}\right)=x^{-2} \nabla(x N) .
$$

By (D.4) we have

$$
\nabla_{(i} Y_{j)}=x^{-1} \nabla_{i} \nabla_{j} N-N \nabla_{i} \nabla_{j}\left(x^{-1}\right)=x^{-1}\left(\nabla_{i} \nabla_{j} N-N|d x| \frac{2}{g} g\right)+N x^{-2} \bar{\nabla}_{i} \bar{\nabla}_{j} x
$$

then

$$
S(Y)-\operatorname{div} Y g=x^{-1}\left[\nabla \nabla N-\Delta N g+(n-1) N|d x|_{\bar{g}}^{2} g+N\left(x^{-1} \bar{\nabla} \nabla x-x^{-1} \Delta_{\bar{g}} x \bar{g}\right)\right] .
$$


On the other hand we have

$$
\begin{aligned}
\operatorname{Ric} g & =\operatorname{Ric} \bar{g}+x^{-1}[(n-2) \overline{\nabla \nabla} x+(\bar{\Delta} x) \bar{g}]-(n-1)|d x| \frac{2}{g} x^{-2} \bar{g} \\
& =-(n-1)|d x|_{\bar{g}} g+\text { l.o. }
\end{aligned}
$$

Finally, we obtain

$$
S(Y)-\operatorname{div} Y g=x^{-1}[\nabla \nabla N-\Delta N g-N(\operatorname{Ric} g+\text { l.o. })] .
$$

Now, we use the inequality

$$
|S(Y)-\operatorname{tr} S(Y) g|^{2}=|S(Y)|^{2}+(n-2)(\operatorname{tr} S(Y))^{2} \geqslant|S(Y)|^{2},
$$

and Proposition D.12 with $s$ there replaced by $s+1$ yields

$$
\int x^{2 s}\left(|\nabla \nabla N-\Delta N g-N \operatorname{Ric} g|^{2}+o(1) N^{2}\right) \geqslant C \int x^{2 s-2}|\nabla(x N)|^{2} .
$$

The result follows now from the following calculation, where Proposition C.7 with $s$ there equal to $s+1$ is used when going from the second to the third line:

$$
\begin{aligned}
\left\|x^{s-1} \nabla(x N)\right\|_{L^{2}} & =\varepsilon\left\|x^{s-1} \nabla(x N)\right\|_{L^{2}}+(1-\varepsilon)\left\|x^{s-1} \nabla(x N)\right\|_{L^{2}} \\
& \geqslant \varepsilon\left\|x^{s} \nabla N\right\|_{L^{2}}-\varepsilon\left\|x^{s-1} N \nabla x\right\|_{L^{2}}+(1-\varepsilon)\left\|x^{s-1} \nabla(x N)\right\|_{L^{2}} \\
& \geqslant \varepsilon\left\|x^{s} \nabla N\right\|_{L^{2}}-\varepsilon\left\|x^{s-1} N \nabla x\right\|_{L^{2}}+(1-\varepsilon) c\left\|x^{s} N\right\|_{L^{2}} \\
& \geqslant C\left(\left\|x^{s} \nabla N\right\|_{L^{2}}+\left\|x^{s} N\right\|_{L^{2}}\right)
\end{aligned}
$$

MÉMOIRES DE LA SMF 94 


\section{APPENDIX E}

\section{POINCARÉ CHARGES}

Let $\mathscr{S}$ be an $n$-dimensional spacelike hypersurface in a $n+1$-dimensional Lorentzian space-time $(\mathscr{M}, g), n \geqslant 2$. Suppose that $\mathscr{M}$ contains an open set $\mathscr{U}$ with a global time coordinate $t$ (with range not necessarily equal to $\mathbb{R}$ ), as well as a global "radial" coordinate $r \in[R, \infty)$, leading to local coordinate systems $\left(t, r, v^{A}\right)$, with $\left(v^{A}\right)$ - local coordinates on some compact $n-1$ dimensional manifold $M$. We further require that $\mathscr{S} \cap \mathscr{U}=\{t=0\}$. Assume that the metric $g$ approaches (as $r$ tends to infinity, in a sense which is made precise below) a background metric $b$. The Hamiltonian analysis of vacuum general relativity in [18] (see also [29, Section 5] or [28, Appendix A]) leads to the following expression for the Hamiltonian associated to the flow of a vector field $X$, assumed to be a Killing vector field of the background $b:^{(1)}$

$$
H(\mathscr{S}, g, b, X)=\frac{1}{2} \int_{\partial \mathscr{S}} \mathbb{U}^{\alpha \beta} d S_{\alpha \beta},
$$

$$
\begin{aligned}
\mathbb{U}^{\nu \lambda} & =\mathbb{U}_{\beta}^{\nu \lambda} X^{\beta}+\frac{1}{8 \pi}\left(\sqrt{\left|\operatorname{det} g_{\rho \sigma}\right|} g^{\alpha[\nu}-\sqrt{\left|\operatorname{det} b_{\rho \sigma}\right|} b^{\alpha[\nu}\right) X_{; \alpha}^{\lambda]}, \\
\mathbb{U}^{\nu \lambda} & =\frac{2\left|\operatorname{det} b_{\mu \nu}\right|}{16 \pi \sqrt{\left|\operatorname{det} g_{\rho \sigma}\right|}} g_{\beta \gamma}\left(e^{2} g^{\gamma[\nu} g^{\lambda] \kappa}\right)_{; \kappa}, \\
e & =\sqrt{\left|\operatorname{det} g_{\rho \sigma}\right|} / \sqrt{\left|\operatorname{det} b_{\mu \nu}\right|} .
\end{aligned}
$$

(The question of convergence of the right-hand-side of (E.1) will be considered shortly. The last term in (E.2) is actually identically zero for asymptotically Euclidean hypersurfaces, but does not vanish for hyperboloidal hypersurfaces and is necessary there to ensure convergence of the integral.) The form (E.1) is most convenient when trying

(1) The integral over $\partial \mathscr{S}$ should be understood by a limiting process, as the limit as $R$ tends to infinity of integrals over the sets $t=0, r=R . \quad d S_{\alpha \beta}$ is defined as $\left.\left.\frac{\partial}{\partial x^{\alpha}}\right\lrcorner \frac{\partial}{\partial x^{\beta}}\right\lrcorner d x^{0} \wedge \cdots \wedge d x^{n}$, with $\lrcorner$ denoting contraction; $g$ stands for the space-time metric unless explicitly indicated otherwise. Further, a semicolon denotes covariant differentiation with respect to the background metric $b$. 
to establish formulae such as (E.14) below, expressing the Poincaré-covariance of the Hamiltonians.

\section{E.1. Initial data asymptotically flat in spacelike directions}

Consider, to start with, Lorentzian metrics which are asymptotically flat in the following sense: there exists a coordinate system $x^{\mu}$ covering a set which contains

$$
\mathscr{S}_{0}:=\left\{x^{0}=0, r(x):=\sqrt{\sum\left(x^{i}\right)^{2}}>R\right\},
$$

and assume that the tensors $g_{\mu \nu}:=g\left(\partial_{\mu}, \partial_{\nu}\right)$ and $b_{\mu \nu}:=b\left(\partial_{\mu}, \partial_{\nu}\right)$ satisfy along $\mathscr{S}_{0}$

$$
\begin{aligned}
b_{\mu \nu} & =\eta_{\mu \nu}:=\operatorname{diag}(-1,+1, \ldots,+1), \\
\left|g_{\mu \nu}-b_{\mu \nu}\right| & \leqslant C r^{-\alpha},\left|\partial_{\sigma} g_{\mu \nu}\right| \leqslant C r^{-\alpha-1}, \quad n / 2-1<\alpha \leqslant n-2 .
\end{aligned}
$$

If one further assumes that the energy-momentum tensor $T_{\mu \nu}$ of $g$ is in $L^{1}\left(\mathscr{S}_{0}\right)$, then the ADM energy-momentum vector defined as

$$
p_{\mu}\left(\mathscr{S}_{0}\right):=H\left(\mathscr{S}_{0}, g, b, \partial_{\mu}\right)
$$

is finite and well defined $[\mathbf{7}, \mathbf{1 9}, \mathbf{2 2}]$. The finiteness of the Lorentz charges,

$$
J_{\mu \nu}\left(\mathscr{S}_{0}\right):=H\left(\mathscr{S}_{0}, g, b, x_{\mu} \partial_{\nu}-x_{\nu} \partial_{\mu}\right),
$$

where $x_{\mu}:=\eta_{\mu \nu} x^{\nu}$, requires further restrictions - there are various ways to proceed $[\mathbf{1 1}, \mathbf{2 0}, \mathbf{5 6}, \mathbf{6 2}]$, the following is convenient for our purposes: let $\Omega \subset \mathbb{R}^{1, n}$ be invariant under the transformation

$$
x^{\mu} \longrightarrow-x^{\mu},
$$

for any $f: \Omega \rightarrow \mathbb{R}$ we set

$$
f^{+}(x)=\frac{1}{2}(f(x)+f(-x)), \quad f^{-}(x)=\frac{1}{2}(f(x)-f(-x)) .
$$

We shall henceforth only consider metrics defined on domains of coordinate systems which are invariant under (E.8), and we will assume that in addition to (E.5) we have

$$
\left|g_{\mu \nu}^{-}\right| \leqslant C(1+r)^{-\alpha_{-}},\left|\partial_{\sigma}\left(g_{\mu \nu}^{-}\right)\right| \leqslant C(1+r)^{-1-\alpha_{-}}, \quad \alpha_{-}>\alpha, \alpha+\alpha_{-}>n-1
$$

We note that in dimension $n+1=3+1$, Equations (E.5) and (E.8) hold for the Schwarzschild metric in the usual static coordinates, with $\alpha=1$ and $\alpha_{-}$- as large as desired. Similarly (E.5), (E.8) hold for the Kerr metric in the Boyer-Lindquist coordinates, discussed in Section F below, with $\alpha=1$ and $\alpha_{-}=2$.

Recall that a boost-type domain $\Omega_{R, T, \theta} \subset \mathbb{R}^{1, n}$ is defined as

$$
\Omega_{R, T, \theta}:=\{r>R,|t|<\theta r+T\},
$$

with $\theta \in(0, \infty]$. We have the following: 
Proposition E.1. - Let $g_{\mu \nu}$ be a Lorentzian metric satisfying (E.5) and (E.9) on a boost-type domain $\Omega_{R, T, \theta}$, and suppose that the coordinate components $\mathscr{T}_{\mu \nu}:=$ $\mathscr{T}\left(\partial_{\mu}, \partial_{\nu}\right)$ of the energy-momentum tensor density,

$$
\mathscr{T}_{\mu \nu}:=\frac{\sqrt{\left|\operatorname{det} g_{\alpha \beta}\right|}}{8 \pi}\left(\operatorname{Ric}_{\mu \nu}-\frac{1}{2} \operatorname{tr}_{g} \operatorname{Ric} g_{\mu \nu}\right),
$$

satisfy

$$
\left|\mathscr{T}_{\mu \nu}\right| \leqslant C(1+r)^{-n-\varepsilon}, \quad\left|\mathscr{T}_{\mu \nu}^{-}\right| \leqslant C(1+r)^{-n-1-\varepsilon}, \quad \varepsilon>0 .
$$

Let $\mathscr{S} \subset \Omega_{R, T, \theta}$ be the hypersurface $\left\{y^{0}=0\right\} \cap \Omega_{R, T, \theta}$, where the coordinates $y^{\mu}$ are obtained from the $x^{\mu}$ 's by a Poincaré transformation,

$$
x^{\mu} \longrightarrow y^{\mu}:=\Lambda_{\nu}^{\mu} x^{\nu}+a^{\mu},
$$

so that $\Lambda^{\mu}{ }_{\nu}$ is a constant-coefficients Lorentz matrix, and $a^{\mu}$ is a set of constants, set $\mathscr{S}_{0}:=\left\{x^{0}=0\right\}$. Then:

(1) The integrals defining the "Poincaré charges" (E.6)-(E.7) of $\mathscr{S}$ and $\mathscr{S}_{0}$ converge.

(2) We have

$$
\begin{aligned}
\left(p_{\mu}(\mathscr{S}), J_{\mu \nu}(\mathscr{S})\right)=\left(\Lambda_{\mu}{ }^{\alpha} p_{\alpha}\left(\mathscr{S}_{0}\right), \Lambda_{\mu}{ }^{\alpha} \Lambda_{\nu}{ }^{\beta} J_{\alpha \beta}\left(\mathscr{S}_{0}\right)\right. & \\
& \left.+a_{\mu} \Lambda_{\nu}{ }^{\alpha} p_{\alpha}\left(\mathscr{S}_{0}\right)-a_{\nu} \Lambda_{\mu}{ }^{\alpha} p_{\alpha}\left(\mathscr{S}_{0}\right)\right) .
\end{aligned}
$$

Here $\Lambda_{\alpha}{ }^{\beta}:=\eta_{\alpha \mu} \Lambda^{\mu}{ }_{\nu} \eta^{\nu \beta}$ and $p_{\mu}\left(\mathscr{S}_{0}\right)=H\left(\mathscr{S}_{0}, g, b, \partial / \partial x^{\mu}\right)$, while $p_{\mu}(\mathscr{S})=$ $H\left(\mathscr{S}, g, b, \partial / \partial y^{\mu}\right)$, similarly for $J_{\mu \nu}$.

Proof. - We have [18]

$$
\int_{\left\{x^{0}=0, r=R\right\}} \mathbb{U}^{\alpha \beta} d S_{\alpha \beta}=2 \int_{\left\{x^{0}=0, R_{0} \leqslant r \leqslant R\right\}} \stackrel{\circ}{\nabla_{\beta}} \mathbb{U}^{\alpha \beta} d S_{\alpha}+\int_{\left\{x^{0}=0, r=R_{0}\right\}} \mathbb{U}^{\alpha \beta} d S_{\alpha \beta},
$$

with

$$
16 \pi \stackrel{\circ}{\nabla}_{\beta} \mathbb{U}^{\alpha \beta}=\mathscr{T}_{\beta}^{\alpha} X^{\beta}+\sqrt{|\operatorname{det} b|}\left(Q^{\alpha}{ }_{\beta} X^{\beta}+Q^{\alpha \beta} \gamma \stackrel{\circ}{\nabla}_{\beta} X^{\gamma}\right),
$$

where $Q^{\alpha}{ }_{\beta}$ is a quadratic form in $\stackrel{\circ}{\nabla}_{\sigma} g_{\mu \nu}$, and $Q^{\alpha \beta}{ }_{\gamma}$ is bilinear in $\stackrel{\circ}{\nabla}_{\sigma} g_{\mu \nu}$ and $g_{\mu \nu}-b_{\mu \nu}$, both with bounded coefficients which are constants plus terms $O\left(r^{-\alpha}\right)$. For $p_{\mu}$ and for $R \geqslant R_{0}$ one immediately obtains

$$
\begin{aligned}
\int_{\left\{x^{0}=0, r=R\right\}} \mathbb{U}^{\alpha \beta} d S_{\alpha \beta} & =\int_{\left\{x^{0}=0, r=R_{0}\right\}} \mathbb{U}^{\alpha \beta} d S_{\alpha \beta}+O\left(R_{0}^{n-2-2 \alpha}\right) \\
& +\frac{1}{8 \pi} \int_{\left\{x^{0}=0, R_{0} \leqslant r \leqslant R\right\}} \mathscr{T}^{\alpha}{ }_{\beta} X^{\beta} d S_{\alpha} \\
& =\int_{\left\{x^{0}=0, r=R_{0}\right\}} \mathbb{U}^{\alpha \beta} d S_{\alpha \beta}+O\left(R_{0}^{n-2-2 \alpha}\right)+O\left(R_{0}^{-\varepsilon}\right) .
\end{aligned}
$$


For $J_{\mu \nu}$ simple parity considerations lead instead to

$$
\int_{\left\{x^{0}=0, r=R\right\}} \mathbb{U}^{\alpha \beta} d S_{\alpha \beta}=\int_{\left\{x^{0}=0, r=R_{0}\right\}} \mathbb{U}^{\alpha \beta} d S_{\alpha \beta}+O\left(R_{0}^{n-1-\alpha-\alpha_{-}}\right)+O\left(R_{0}^{-\varepsilon}\right) .
$$

Passing to the limit $R \rightarrow \infty$ one obtains convergence of $p_{\mu}\left(\mathscr{S}_{0}\right)$ and of $J_{\mu \nu}\left(\mathscr{S}_{0}\right)$. For further reference we note the formulae

$$
\begin{aligned}
p_{\mu}\left(\mathscr{S}_{0}\right) & =\int_{\left\{x^{0}=0, r=R_{0}\right\}} \mathbb{U}^{\alpha \beta} d S_{\alpha \beta}+\frac{1}{16 \pi} \int_{r \geqslant R_{0}} \mathscr{T}^{\mu}{ }_{\nu} X^{\nu} d S_{\mu}+O\left(R_{0}^{n-2-2 \alpha}\right), \\
J_{\mu \nu}\left(\mathscr{S}_{0}\right) & =\int_{\left\{x^{0}=0, r=R_{0}\right\}} \mathbb{U}^{\alpha \beta} d S_{\alpha \beta}+\frac{1}{16 \pi} \int_{r \geqslant R_{0}} \mathscr{T}_{\nu}^{\mu} X^{\nu} d S_{\mu}+O\left(R_{0}^{n-1-\alpha-\alpha-}\right) .
\end{aligned}
$$

Because Lorentz transformations commute with the antipodal map (E.8) the boundary conditions (E.5) and (E.9) are preserved under them, and convergence of the Poincaré charges of $\mathscr{S}$ for transformations of the form (E.13) with $a^{\mu}=0$ follows. In order to establish point (2), still for $a^{\mu}=0$, we use Stokes' theorem on a set $\mathscr{T}_{R}$ defined as

$$
\mathscr{T}_{R}=\left\{r=R, 0 \leqslant t \leqslant-\left(\Lambda^{0}{ }_{0}\right)^{-1} \Lambda^{0}{ }_{i} x^{i}\right\} \cup\left\{r=R, 0 \geqslant t \geqslant-\left(\Lambda^{0}{ }_{0}\right)^{-1} \Lambda^{0}{ }_{i} x^{i}\right\},
$$

so that the boundary $\partial \mathscr{T}_{R}$ has two connected components, the set $\mathscr{S}_{0} \cap\{r=R\}$ and the set $\mathscr{S} \cap\{r=R\}$. This leads to

$$
\int_{\mathscr{S} \cap\{r=R\}} \mathbb{U}^{\alpha \beta} d S_{\alpha \beta}=2 \int_{\mathscr{T}_{R}} \stackrel{\circ}{\nabla}_{\beta} \mathbb{U}^{\alpha \beta} d S_{\alpha}+\int_{\mathscr{S}_{0} \cap\{r=R\}} \mathbb{U}^{\alpha \beta} d S_{\alpha \beta},
$$

The boundary conditions ensure that the integral over $\mathscr{T}_{R}$ vanishes in the limit $R \rightarrow$ $\infty$ (for $p_{\mu}$ this is again straightforward, while for $J_{\mu \nu}$ this follows again by parity considerations), so that

$$
H(\mathscr{S}, g, b, X)=H\left(\mathscr{S}_{0}, g, b, X\right) .
$$

We consider finally a translation; Stokes' theorem on the $n$-dimensional region

$$
\left\{y^{\mu}=x^{\mu}+s a^{\mu}, s \in[0,1], x^{\mu} \in \mathscr{S}, r\left(x^{\mu}\right)=R\right\}
$$

leads again — in the limit $R \rightarrow \infty$ - to (E.22), in particular $H(\mathscr{S}, g, b, X)$ converges. The transformation law (E.14) follows now from (E.22) by the following calculation:

$$
\begin{aligned}
J_{\mu \nu}(\mathscr{S}) & :=H\left(\mathscr{S}, g, b, y_{\mu} \frac{\partial}{\partial y^{\nu}}-y_{\nu} \frac{\partial}{\partial y^{\mu}}\right) \\
& =H\left(\mathscr{S}_{0}, g, b, y_{\mu} \frac{\partial}{\partial y^{\nu}}-y_{\nu} \frac{\partial}{\partial y^{\mu}}\right) \\
& =H\left(\mathscr{S}_{0}, g, b,\left(\Lambda_{\mu}{ }^{\alpha} x_{\alpha}+a_{\mu}\right) \Lambda_{\nu}{ }^{\beta} \frac{\partial}{\partial x^{\beta}}-\left(\Lambda_{\nu}{ }^{\alpha} x_{\alpha}+a_{\nu}\right) \Lambda_{\mu}{ }^{\beta} \frac{\partial}{\partial x^{\beta}}\right) .
\end{aligned}
$$

It is convenient to have a initial data version of (E.1), in the asymptotically flat vacuum case this is easily implemented as follows: let $(\mathscr{S}, K, g)$ be an asymptotically flat vacuum initial data set, if the data are sufficiently differentiable there exists a vacuum development $\left(M,{ }^{n+1} g\right)$ of the data so that $\mathscr{S}$ can be isometrically identified with a hypersurface $t=0$ in $M$, with $K$ corresponding to the second fundamental 
form of $\mathscr{S}$ in $\left(M,{ }^{n+1} g\right)$. We can introduce Gauss coordinates around $\mathscr{S}$ to bring ${ }^{n+1} \mathrm{~g}$ to the form

$$
{ }^{n+1} g=-d t^{2}+g_{t}
$$

where $g_{t}$ is a family of Riemannian metrics on $\mathscr{S}$ with $g_{0}=g$. We then set

$$
b=-d t^{2}+e,
$$

where $e$ is the Euclidean flat metric equal to $\operatorname{diag}(+1, \ldots,+1)$ in asymptotically flat coordinates on $\mathscr{S}$. Let $n_{b}$ be the future directed $b$-unit normal to $\mathscr{S}$ and let $(Y, N)$ be the KID determined on $\mathscr{S}$ by the $b$-Killing vector $X$; by definition,

$$
X=N n_{b}+Y, b\left(n_{b}, Y\right)=0 \text { along } \mathscr{S} \text {. }
$$

Since the future pointing $g$-unit normal to $\mathscr{S}$, say $n_{g}$, coincides with $n_{b}$, we also have

$$
X=N n_{g}+Y, g\left(n_{g}, Y\right)=0 .
$$

We define the Poincaré charges $Q$ by the formula

$$
Q((Y, N),(K, g)):=H\left(\mathscr{S},{ }^{n+1} g, b, V N+Y\right) .
$$

It is well known that the integrand of (E.25) can be expressed in terms of $K, g$, as well as the first derivatives of $g$. The initial data form of (E.17) reads

$$
\begin{aligned}
\int_{\left\{x^{0}=0, r=R\right\}} \mathbb{U}^{\alpha \beta} d S_{\alpha \beta}=\int_{\left\{x^{0}=0, r=R_{0}\right\}} & \mathbb{U}^{\alpha \beta} d S_{\alpha \beta} \\
& +\frac{1}{8 \pi} \int_{\left\{x^{0}=0, R_{0} \leqslant r \leqslant R\right\}}\left(Y^{i} J_{i}+N \rho+q\right) d \mu_{g},
\end{aligned}
$$

where $q$ is a quadratic form in $g_{i j}-\delta_{i j}, \partial_{k} g_{i j}$, and $K_{i j}$, with uniformly bounded coefficients whenever $g_{i j}$ and $g^{i j}$ are uniformly bounded. This follows immediately from (E.15)-(E.16), together with the $n+1$ decomposition of the energy-momentum tensor density (E.11), and of the error term in (E.16). One can also work directly with the initial data equivalents of the boundary integrals in (E.26) - cf., e.g., [11] - but those are somewhat cumbersome when studying behavior of the charges under Lorentz transformations. 



\section{APPENDIX F}

\section{THE REFERENCE FAMILY OF KERR METRICS}

Let us denote by $\mathscr{K}_{i^{0}}$ the family of Cauchy data $(g, K)$ obtained as follows: let ${ }^{4} g$ be a Kerr metric with $m \neq 0, a \in \mathbb{R}$; in Boyer-Lindquist coordinates $(t, r, \theta, \varphi)$ we have [53, p. 100] (see also http://grdb.org)

$$
\begin{gathered}
{ }^{4} g_{t t}=-1+\frac{2 m r}{\rho^{2}}, \quad{ }^{4} g_{t \varphi}=-\frac{2 m r a \sin ^{2} \theta}{\rho^{2}}, \quad{ }^{4} g_{r r}=\frac{\rho^{2}}{\triangle}, \quad{ }^{4} g_{\theta \theta}=\rho^{2}, \\
{ }^{4} g_{\varphi \varphi}=\sin ^{2} \theta\left(r^{2}+a^{2}+\frac{2 m r a^{2} \sin ^{2} \theta}{\rho^{2}}\right),
\end{gathered}
$$

where

$$
\rho^{2}=r^{2}+a^{2} \cos ^{2} \theta, \quad \triangle=r^{2}-2 m r+a^{2} .
$$

Introduce a "quasi-Minkowskian" coordinate system $\left(x^{\mu}\right)=\left(t, x^{i}\right)$ by setting

$$
x^{1}=r \sin \theta \cos \varphi, \quad x^{2}=r \sin \theta \sin \varphi, \quad x^{3}=r \cos \theta,
$$

which brings ${ }^{4} g_{\mu \nu}$ to the form $\eta_{\mu \nu}+O\left(r^{-1}\right)$ for $x^{\mu}$ 's in a set $r \geqslant R_{0}$ for some $R_{0}$, and apply to it a Poincaré transformation (E.13). We further assume that $\left(\Lambda^{\mu}{ }_{\nu}, a^{\mu}\right)$ belongs to the connected component $G_{0}$ of the identity of the Poincaré group. Then $(g, K)$ are defined on the set

$$
\left\{\sqrt{\sum_{i}\left(y^{i}\right)^{2}}>R\right\}
$$

for some $R=R\left(m, a, \Lambda^{\mu}{ }_{\nu}, a^{\mu}\right)$ by extracting the gravitational initial data out of the metric ${ }^{4} g$ on the hypersurface $y^{0}=0$. The function $R\left(m, a, \Lambda_{\nu}^{\mu}, a^{\mu}\right)$ can be chosen to be continuous, in particular for any set $\left(m_{0}, a_{0}, \Lambda_{0}{ }^{\mu}{ }_{\nu}, a_{0}^{\mu}\right)$ there exists a neighborhood $\mathscr{O}_{0}$ thereof such that $R\left(m, a, \Lambda^{\mu}{ }_{\nu}, a^{\mu}\right)$ can be chosen independently of $\left(m, a, \Lambda^{\mu}{ }_{\nu}, a^{\mu}\right) \in \mathscr{O}_{0}$. We equip $\mathscr{K}_{i^{0}}$ with the topology of uniform convergence on relatively compact open sets; any weighted Sobolev topology on the set of initial data will lead, by restriction, to this topology on $\mathscr{K}_{i^{0}}$. 
We wish to show that the set $\mathscr{K}_{i^{0}}$ can be uniquely parameterized ${ }^{(1)}$ by the Poincaré charges $\left(p_{\mu}, J_{\mu \nu}\right)$ defined in (E.6)-(E.7), with $p_{\mu}$ ranging over the set of timelike vectors $I(0)$ in the Minkowski space-time $\mathbb{R}^{1,3}$, and $J_{\mu \nu}$ ranging over all anti-symmetric twocovariant tensors. In other words:

Proposition F.1. - The map

$$
Q: \mathscr{K}_{i^{0}} \ni(g, K) \longrightarrow\left(p_{\mu}, J_{\mu \nu}\right) \in I(0) \times \mathbb{R}^{6} \subset \mathbb{R}^{4} \times \mathbb{R}^{6}
$$

is a continuous bijection.

Proof. - Let $(g, K)$ be the Cauchy data on $\left\{x^{0}=0, r \geqslant R_{0}\right\}$ for a Kerr metric as above with some parameters $m \in \mathbb{R}^{*}$ and $a \in \mathbb{R}$, we then have

$$
p_{\mu}=(m, 0,0,0), J_{\mu \nu}=2 m a \delta_{[\mu}^{1} \delta_{\nu]}^{2} .
$$

The transformation law (E.14) shows that for any vector $n^{i} \in \mathbb{R}^{3}$ satisfying $\sum_{i}\left(n^{i}\right)^{2}=1$ we can obtain a pair $\left(p_{\mu}, J_{\mu \nu}\right)$ of the form

$$
p_{\mu}=m \delta_{\mu}^{0}, \quad J_{0 i}=0, J_{i j}= \pm m a \varepsilon_{i j k} n^{k},
$$

by

- either performing a rotation by an angle less than or equal to $\pi / 2$ in the plane $\operatorname{Span}\left(\partial_{z}, n^{i} \partial_{i}\right)$ which brings $n^{i} \partial_{i}$ to $\partial_{z}$, then we choose the sign + , or

- we perform a rotation by an angle less than or equal to $\pi / 2$ in the plane $\operatorname{Span}\left(\partial_{z}, n^{i} \partial_{i}\right)$ which brings $n^{i} \partial_{i}$ to $-\partial_{z}$, then we choose the sign -.

In the overlapping case $n^{i} \partial_{i} \perp \partial_{z}$ the choice does not matter because the resulting metrics (and thus initial data) are identical "modulo gauge" - the corresponding transformation $a \rightarrow-a,(t, r, \varphi, \theta) \rightarrow(t, r,-\varphi, \pi-\theta)$ is an isometry of the Kerr metric. Next, a space-translation $a^{i} \in \mathbb{R}^{3}$ produces out of (F.5) a pair $\left(p_{\mu}, J_{\mu \nu}\right)$

$$
p_{\mu}=m \delta_{\mu}^{0}, \quad J_{0 i}=-m a_{i}, J_{i j}= \pm m a \varepsilon_{i j k} n^{k} .
$$

It follows that any set $\left(p_{\mu}=m \delta_{\mu}^{0}, J_{\mu \nu}\right)$ can be obtained in a unique way by calculating the charges (E.6)-(E.7) using initial data in $\mathscr{K}_{i^{0}}$ by the operations just described. Now, for any timelike $p_{\mu}$ there exists precisely one boost transformation $\Lambda^{\mu}{ }_{\nu}$ in the plane $\operatorname{Span}\left(\delta_{\mu}^{0}, p_{\mu}\right)$ which maps $m \delta_{\mu}^{0}$ to $p_{\mu}$, provided $m$ is suitably chosen, and we conclude by noting that, at fixed $\Lambda^{\mu}{ }_{\nu}$, the map

$$
\mathbb{R}^{6} \ni J_{\mu \nu} \longrightarrow \Lambda_{\mu}{ }^{\alpha} \Lambda_{\nu}{ }^{\beta} J_{\alpha \beta} \in \mathbb{R}^{6}
$$

is a linear isomorphism.

${ }^{(1)}$ The construction of the set $\mathscr{K}_{i 0}$ involves twelve free parameters, however two of them are redundant because of the existence of the two-parameter group of isometries of the Kerr metric. 
We end this section by verifying that the initial data for the Kerr metric in BoyerLindquist coordinates are parity symmetric. First, we note that $g_{i j}$ is obviously even. Next, we have

$$
{ }^{4} g_{t i} d x^{i}={ }^{4} g_{t \varphi} d \varphi=-\frac{2 m r a \sin ^{2} \theta}{\rho^{2}} d \varphi=-\frac{2 m a}{r \rho^{2}}(x d y-y d x),
$$

so that the coordinate components of the shift vector are odd. Now, the lapse function is symmetric under parity. Further, the derivatives of an even function are odd and vice-versa; in particular the Christoffel symbols are odd while the partial derivatives of the coordinate components of the shift vector are even. The usual formula for $K_{i j}$ in terms of the derivatives of the shift vector yields the result. 



\section{APPENDIX G}

\section{UNIFORM LOCAL INVERTIBILITY}

Proposition G.1. - Let $\left(V_{x},\|\cdot\|_{V_{x}}\right)_{x \in A}$ and $\left(W_{x},\|\cdot\|_{W_{x}}\right)_{x \in A}$ be two families of Banach spaces. Let $r>0$ and let $\left\{f_{x}: B_{V_{x}}(0, r) \rightarrow W_{x}\right\}_{x \in A}$ be a family of differentiable functions such that:

(1) $D f_{x}(0): V_{x} \rightarrow W_{x}$ has a right inverse for all $x \in A$ which is bounded independently of $x \in A$.

(2) $\left\|f_{x}(v+h)-f_{x}(v)-D f_{x}(v) h\right\|_{W_{x}} /\|h\|_{V_{x}}^{2}$ is bounded independently of $x \in A$, $v \in B_{V_{x}}(0, r)$ and $h \in V$ such that $v+h \in B_{V_{x}}(0, r)$.

(3) $\left\|D f_{x}(v+h)-D f_{x}(v)\right\|_{L\left(V_{x}, W_{x}\right)} /\|h\|_{V_{x}}$ is bounded independently of $x \in A$, $v \in B_{V_{x}}(0, r)$ and $h \in V$ such that $v+h \in B_{V_{x}}(0, r)$.

Then there exists $\varepsilon>0$ and $C>0$ such that for all $x \in A$ and all $\delta f \in W_{x}$, $\|\delta f\|_{W_{x}}<\varepsilon$, there exists a solution $\delta x \in V_{x}$ of the equation

$$
f_{x}(\delta x)-f_{x}(0)=\delta f
$$

which satisfies $\|\delta x\|_{V_{x}} \leqslant C\|\delta f\|_{W_{x}}$.

Proof. - From 1), there exist a constant $C_{1}$ such that for all $x \in A$ and all $w \in W_{x}$, the equation

$$
D f_{x}(0) h=w,
$$

has a solution $h \in V_{x}$ such that

$$
\|h\|_{V_{x}} \leqslant C_{1}\|w\|_{W_{x}} .
$$

From 2) and 3), there exist constants $C_{2}$ and $C_{3}$ such that for all $x \in A$, all $v \in$ $B_{V_{x}}(0, r)$ and all $h \in V$,

$$
\begin{aligned}
\left\|f_{x}(v+h)-f_{x}(v)-D f_{x}(v) h\right\|_{W_{x}} & \leqslant C_{2}\|h\|_{V_{x}}^{2}, \\
\left\|D f_{x}(v+h)-D f_{x}(v)\right\|_{L\left(V_{x}, W_{x}\right)} & \leqslant C_{3}\|h\|_{V_{x}} .
\end{aligned}
$$

Let $x \in A$ and $\delta f \in W_{x}$. We will construct a Picard sequence $\left\{h_{n}\right\}$ such that $\sum h_{n}$ converges to a solution when $\delta f$ is small enough. From 1), we have a solution $h_{0} \in V_{x}$ 
of

$$
D f_{x}(0) h_{0}=\delta f
$$

which satisfies $\left\|h_{0}\right\|_{V_{x}} \leqslant C_{1}\|\delta f\|_{W_{x}}$. Let $\delta x_{1}:=h_{0}$ which is in $B_{V_{x}}(0, r)$ if $\|\delta f\|_{W_{x}}$ is small enough. Let us now define the sequence $h_{i+1}$, solution of

$$
D f_{x}(0) h_{i+1}=f_{x}(0)-f_{x}\left(\delta x_{i+1}\right)+\delta f
$$

where $\delta x_{i+1}=\delta x_{i}+h_{i}$ (we assume that $\delta x_{i+1} \in B_{V_{x}}(0, r)$, it will be justified at the end of the proof). We have that

$$
\begin{aligned}
D f_{x}(0) h_{i+1}= & f_{x}(0)-f_{x}\left(\delta x_{i}\right)+f_{x}\left(\delta x_{i}\right)-f_{x}\left(\delta x_{i+1}\right)+\delta f \\
= & D f_{x}(0) h_{i}-\left[f_{x}\left(\delta x_{i+1}\right)-f_{x}\left(\delta x_{i}\right)\right] \\
= & \sum_{p=0}^{i-1}\left[D f_{x}\left(\delta x_{p}\right)-D f_{x}\left(\delta x_{p+1}\right)\right] h_{i} \\
& \quad+\left[D f_{x}\left(\delta x_{i}\right) h_{i}+f_{x}\left(\delta x_{i}\right)-f_{x}\left(\delta x_{i}+h_{i}\right)\right],
\end{aligned}
$$

with $\delta x_{0}=0$, so by hypothesis 1 ), 2) and 3 ), we have

$$
\left\|h_{i+1}\right\|_{V_{x}} \leqslant C_{1}\left(\sum_{p=0}^{i-1} C_{3}\left\|h_{p}\right\|_{V_{x}}\left\|h_{i}\right\|_{V_{x}}+C_{2}\left\|h_{i}\right\|_{V_{x}}^{2}\right) .
$$

Let $K:=\max \left(C_{1} C_{2}, C_{1} C_{3}\right)$, then we have

$$
\left\|h_{i+1}\right\|_{V_{x}} \leqslant K\left\|h_{i}\right\|_{V_{x}} \sum_{p=0}^{i}\left\|h_{p}\right\|_{V_{x}} .
$$

Choose any $\delta \in] 0,1[$, let $\varepsilon$ be small enough so that

$$
K C_{1} \varepsilon<1, \quad \frac{\left(K C_{1} \varepsilon\right)^{1-\delta}}{1-\left(K C_{1} \varepsilon\right)^{\delta}} \leqslant 1
$$

and such that for all $t \in[0, \varepsilon[$,

$$
\frac{C_{1} t}{1-\left(K C_{1} t\right)^{\delta}} \leqslant 2 C_{1} t<r
$$

and let $C:=2 C_{1}$. If $\|\delta f\|_{W_{x}} \leqslant \varepsilon$, from Lemma G.3 with $a_{i}=\left\|h_{i}\right\|_{V_{x}}$, the sequence $\delta x_{n+1}:=\sum_{i=0}^{n} h_{i}$ is convergent in $V_{x}$ to a limit $\delta x$ which satisfies

$$
\|\delta x\|_{V_{x}} \leqslant \frac{\left\|h_{0}\right\|_{V_{x}}}{1-\left(K\left\|h_{0}\right\|_{V_{x}}\right)^{\delta}} \leqslant \frac{C_{1}\|\delta f\|_{W_{x}}}{1-\left(K C_{1}\|\delta f\|_{W_{x}}\right)^{\delta}} \leqslant C\|\delta f\|_{W_{x}}<r .
$$

Note that for all $n \geqslant 0,\left\|\delta x_{n+1}\right\|_{V_{x}}<r$. On the other hand, as $h_{i+1}$ goes to zero in $V_{x}$ we have that $f_{x}(0)-f_{x}\left(\delta x_{i}\right)-\delta f=D f_{x}(0) h_{i+1}$ goes to zero in $W_{x}$.

The following result is needed to be able to obtain weighted Hölder regularity of the solutions obtained, to start with, in weighted Sobolev spaces. In our applications the spaces $E_{x}$ will be the weighted Hölder spaces $C_{\phi, \varphi}^{k+2, \alpha} \times C_{\phi, \varphi}^{k+2, \alpha}$, the $F_{x}$ 's will be $C_{\phi, \varphi^{\prime}}^{k+1, \alpha} \times C_{\phi, \varphi^{\prime}}^{k, \alpha}$, the $G_{x}$ 's will correspond to $C_{\phi, \varphi^{\prime \prime}}^{k+1, \alpha} \times C_{\phi, \varphi^{\prime \prime}}^{k, \alpha}$, for appropriate weights 
$\varphi, \varphi^{\prime}, \varphi^{\prime \prime}$, see the proof of Proposition 3.13. Finally, $A$ should be thought of as a neighborhood of $x_{0}=\left(K_{0}, g_{0}\right)$ in $\left(C_{\phi, 1}^{k+3, \alpha} \times C_{\phi, 1}^{k+4, \alpha}\right) \cap\left(W_{\phi}^{k+3, \infty} \times W_{\phi}^{k+4, \infty}\right)$.

For the following result we shall denote by

$$
D f_{x}(0)_{r}^{-1}
$$

the right inverse of $D f_{x}(0)$, the existence of which has been assumed in point (1) of the preceding proposition.

Proposition G.2. - Under the hypotheses of Proposition G.1, consider three families of Banach spaces $\left(E_{x},\|\cdot\|_{E_{x}}\right)_{x \in A},\left(F_{x},\|\cdot\|_{F_{x}}\right)_{x \in A}$ and $\left(G_{x},\|\cdot\|_{G_{x}}\right)_{x \in A}$ such that $G_{x}$ is continuously embedded both in $F_{x}$ and in $W_{x}$, with the norms of the embeddings uniformly bounded in $x \in A$. Assume there exist a $r^{\prime}>0$ such that $\left\{f_{x}: B_{E_{x}}\left(0, r^{\prime}\right) \rightarrow F_{x}\right\}_{x \in A}$ is defined, differentiable and verifies:

(1) if $h$ is in the image of $D f_{x}(0)_{r}^{-1}$ and $D f_{x}(0) h \in F_{x}$ then $h \in E_{x}$ and

$$
\|h\|_{E_{x}} \leqslant C\left(\|h\|_{V_{x}}+\left\|D f_{x}(0) h\right\|_{F_{x}}\right),
$$

where $C$ does not depend on $x \in A$.

(2) $\left\|f_{x}(v+h)-f_{x}(v)-D f_{x}(v) h\right\|_{G_{x}} /\|h\|_{E_{x}}^{2}$ is bounded independently of $x \in A$, $v \in B_{E_{x}}\left(0, r^{\prime}\right)$ and $h \in E_{x}$ such that $v+h \in B_{E_{x}}\left(0, r^{\prime}\right)$.

(3) $\left\|D f_{x}(v+h)-D f_{x}(v)\right\|_{L\left(E_{x}, G_{x}\right)} /\|h\|_{E_{x}}$ is bounded independently of $x \in A$, $v \in B_{E_{x}}\left(0, r^{\prime}\right)$ and $h \in V$ such that $v+h \in B_{E_{x}}\left(0, r^{\prime}\right)$.

Then there exists $\varepsilon>0$ and $C^{\prime}>0$ such that for all $x \in A$ and all $\delta f \in W_{x} \cap F_{x}$ satisfying

$$
\|\delta f\|_{W_{x}}+\|\delta f\|_{F_{x}}<\varepsilon
$$

there exists a solution $\delta x \in E_{x}$ satisfying

$$
\|\delta x\|_{E_{x}} \leqslant C^{\prime}\left(\|\delta f\|_{W_{x}}+\|\delta f\|_{F_{x}}\right) .
$$

Proof. - The constant $C$ which appears in the proof may change from term to term and line to line. The solution is constructed by the same method as in the proof of Proposition G.1. Let, thus, $h_{i}$ be the sequence defined there, by hypothesis 1 . for all $i \geqslant-1$ we have $h_{i+1} \in E_{x}$ and

$$
\begin{aligned}
\left\|h_{i+1}\right\|_{E_{x}} & \leqslant C\left(\left\|h_{i+1}\right\|_{V_{x}}+\left\|D f_{x}(0) h_{i+1}\right\|_{F_{x}}\right) \\
& \leqslant C\left(\left\|D f_{x}(0) h_{i+1}\right\|_{W_{x}}+\left\|D f_{x}(0) h_{i+1}\right\|_{F_{x}}\right) \leqslant C\left\|D f_{x}(0) h_{i+1}\right\|_{G_{x}},
\end{aligned}
$$

which is clearly true regardless of whether or not the last term is finite. On the other hand, from equation (G.1) together with the hypotheses 2. and 3. we have that

$$
\left\|D f_{x}(0) h_{i+1}\right\|_{G_{x}} \leqslant C\left\|h_{i}\right\|_{E_{x}} \sum_{k=0}^{i}\left\|h_{k}\right\|_{E_{x}} .
$$


So from Lemma G.3 with $a_{i}=\left\|h_{i}\right\|_{E_{x}}$, if $\delta f$ is sufficiently small in $F_{x}$ norm, then the sequence $\sum_{k=0}^{i} h_{k}$ is convergent in $E_{x}$ to some element $\delta x \in E_{x}$, with

$$
\|\delta x\|_{E_{x}} \leqslant C\left\|h_{0}\right\|_{E_{x}} \leqslant C\left(\left\|h_{0}\right\|_{V_{x}}+\left\|D f_{x}(0) h_{0}\right\|_{F_{x}}\right)=C\left(\|\delta f\|_{W_{x}}+\|\delta f\|_{F_{x}}\right) .
$$

\section{G.1. A sequence adapted to the Picard method}

Lemma G.3. - Let $K>0, \delta \in] 0,1\left[\right.$, and let $\left\{a_{n}\right\}_{n \in \mathbb{N}}$ be a sequence with nonnegative terms which verifies, for all $n>0$,

$$
a_{n+1} \leqslant K a_{n} \sum_{i=0}^{n} a_{i} .
$$

If $a_{0}$ is small enough to verify

$$
K a_{0}<1 \text { and } \frac{\left(K a_{0}\right)^{1-\delta}}{1-\left(K a_{0}\right)^{\delta}} \leqslant 1,
$$

then the sequence $S_{n}\left(a_{0}\right):=\sum_{i=0}^{n} a_{i}$ is convergent to a limit denoted $S\left(a_{0}\right)$ which satisfies

$$
0 \leqslant S_{n}\left(a_{0}\right) \leqslant S\left(a_{0}\right) \leqslant \frac{a_{0}}{1-\left(K a_{0}\right)^{\delta}},
$$

in particular, $S$ is continuous at 0.

Proof. - Let $b_{n}:=K a_{n}$, we have

$$
b_{n+1} \leqslant b_{n} \sum_{i=0}^{n} b_{i} .
$$

We will show by induction that

$$
b_{n} \leqslant b_{0}^{1+n \delta} .
$$

Equation G.2 holds for $n=0$, assume it hold for all integers less than or equal to $n$, we then have

$$
\begin{aligned}
b_{n+1} & \leqslant b_{0}^{1+n \delta} \sum_{i=0}^{n} b_{0}^{1+i \delta} \leqslant b_{0}^{2+n \delta} \frac{1-b_{0}^{(n+1) \delta}}{1-b_{0}^{\delta}} \\
& \leqslant b_{0}^{2+n \delta} \frac{1}{1-b_{0}^{\delta}} \leqslant b_{0}^{1+(n+1) \delta} \frac{b_{0}^{1-\delta}}{1-b_{0}^{\delta}} \\
& \leqslant b_{0}^{1+(n+1) \delta},
\end{aligned}
$$

the last inequality following from the second hypothesis on $a_{0}$. To conclude, it suffices to remark that

$$
0 \leqslant \sum_{i=0}^{n} b_{i} \leqslant \sum_{i=0}^{\infty} b_{i} \leqslant \sum_{i=0}^{\infty} b_{0}^{1+i \delta}=\frac{b_{0}}{1-b_{0}^{\delta}}
$$




\section{APPENDIX H}

\section{SMALL INITIAL DATA ON A BOUNDED DOMAIN IN $\mathbb{R}^{3}$}

Let $\Omega$ be a bounded domain in $\mathbb{R}^{3}$ with smooth boundary, and let $\widetilde{g}$ be any smooth up-to-boundary Riemannian metric on $\bar{\Omega}$ such that

$$
\frac{1}{2} e(X, X) \leqslant \widetilde{g}(X, X) \leqslant 2 e(X, X),
$$

where $e$ is the Euclidean metric. It can be seen that there are no conformal Killing vectors which vanish on $\partial \Omega$ (cf., e.g. [3, Proposition 6.2.2]) which implies that the operator

$$
\stackrel{\circ}{H}_{2} \ni X \longrightarrow \widetilde{D}_{i}\left(\widetilde{D}^{i} X^{j}+\widetilde{D}^{j} X^{i}-\frac{2}{3} \widetilde{D}_{k} X^{k} \widetilde{g}^{i j}\right) \in L^{2}
$$

has no kernel ( $\widetilde{D}$ - the Levi-Civita connection of $\widetilde{g})$, and can thus be used to construct $\widetilde{g}$-transverse $\left(\widetilde{D}_{i} L^{i j}=0\right)$ traceless $\left(\widetilde{g}^{i j} L_{i j}=0\right)$ tensors $L_{i j}$ on $\Omega$ in the usual way. When $\widetilde{g}$ is parity-symmetric, then parity-antisymmetric $L_{i j}$ 's can be obtained by replacing $L_{i j}$ with $\left(L_{i j}(x)-L_{i j}(-x)\right) / 2$. Let, thus, any parity-antisymmetric, transverse, traceless, $L_{i j}$ be given, for $\sigma \in[0,1]$ consider the Lichnerowicz equation:

$$
8 \Delta_{\widetilde{g}} \phi-R(\widetilde{g}) \phi+\sigma^{2}|L|_{\widetilde{g}}^{2} \phi^{-7}=0,
$$

which we rewrite as

$$
L u:=\left(\Delta_{\widetilde{g}}+s\right) u=F(u),
$$

where $u:=\phi-1$, while $\Delta_{\tilde{g}}+s$ is the linearization of $\frac{1}{8}($ H.2) at $\phi=1$,

$$
s:=-\frac{R(\widetilde{g})}{8}-7 \sigma^{2}|L| \frac{2}{g} \text {. }
$$

One will obtain a solution

$$
\left(K_{i j}:=\sigma \phi^{-2} L_{i j}, g_{i j}:=\phi^{4} \widetilde{g}_{i j}\right)
$$

of the vacuum constraint equations using the inverse function theorem in, e.g., weighted Hölder spaces, if one can show that the operator $L$ appearing at the left-hand-side of (H.3) has no kernel. In order to show that this is indeed the case 
for $\widetilde{g}-e$ small enough in $C_{2}(\bar{\Omega})$, and for $\sigma$ small enough, let $C_{P}$ be the constant appearing in the Poincaré inequality for $\Omega$ :

$$
\forall u \in \stackrel{\circ}{H}_{1}(\Omega) \quad \int_{\Omega} u^{2} d^{3} x \leqslant C_{P} \int_{\Omega}|d u|_{e}^{2} d^{3} x
$$

it follows from (H.1) that we also have

$$
\forall u \in \stackrel{\circ}{H}_{1}(\Omega) \quad \int_{\Omega} u^{2} \sqrt{\operatorname{det} \widetilde{g}} d^{3} x \leqslant 4 \sqrt{2} C_{P} \int_{\Omega}|d u|_{\widetilde{g}}^{2} \sqrt{\operatorname{det} \widetilde{g}} d^{3} x
$$

If $L u=0$, by integration by parts one obtains

$$
\int_{\Omega}\left(-\left|d u^{2}\right|_{\tilde{g}}+s u^{2}\right) \sqrt{\operatorname{det} \widetilde{g}} d^{3} x=0,
$$

and the Poincaré inequality gives

$$
\begin{aligned}
\int_{\Omega} u^{2} \sqrt{\operatorname{det} \widetilde{g}} d^{3} x & \leqslant 4 \sqrt{2} C_{P} \int_{\Omega}|d u|_{\tilde{g}}^{2} \sqrt{\operatorname{det} \widetilde{g}} d^{3} x \\
& \leqslant 4 \sqrt{2} C_{P} \sup |s| \int_{\Omega} u^{2} \sqrt{\operatorname{det} \widetilde{g}} d^{3} x,
\end{aligned}
$$

hence $u=0$ if $\|s\|_{L^{\infty}(\Omega)}$ is small enough, and the inverse function theorem applies. Clearly the resulting $(K, g)$ will be non-trivial as soon as $\widetilde{g}$ is not conformally flat.

Let $(m, \vec{p})$ be the ADM four-momentum of $(K, g)$ obtained by integrating $\mathbb{U}$ given by (E.2) (expressed in terms of $g$ and $K$ ) over $\partial \Omega$; here $b$ should be taken as the Minkowski metric, and space coordinates harmonic for $g$ should be used - such coordinates can be found globally on $\Omega$ if $g$ is close enough to $e$. At $\sigma=0$ we have $\vec{p}=0$, while it follows from the calculations in [7] that $m>0$ (choosing $g$ closer to $e$ if necessary). Continuity then shows that choosing $\sigma$ small enough we will obtain

$$
|\vec{p}|_{e} \leqslant \frac{1}{2} m
$$

The initial data set $(K, g)$ will then fulfill all the requirements set forth in Theorem 8.14. 


\section{BIBLIOGRAPHY}

[1] L. Andersson - Elliptic systems on manifolds with asymptotically negative curvature, Indiana Univ. Math. Jour. 42 (1993), p. 1359-1388.

[2] L. Andersson \& P. Chruściel - On "hyperboloidal" Cauchy data for vacuum Einstein equations and obstructions to smoothness of Scri, Commun. Math. Phys. 161 (1994), p. 533-568.

[3] - On asymptotic behavior of solutions of the constraint equations in general relativity with "hyperboloidal boundary conditions", Dissert. Math. 355 (1996), p. 1-100.

[4] L. Andersson, P. Chruściel \& H. Friedrich - On the regularity of solutions to the Yamabe equation and the existence of smooth hyperboloidal initial data for Einsteins field equations, Commun. Math. Phys. 149 (1992), p. 587-612.

[5] T. Aubin - Espaces de Sobolev sur les variétés Riemanniennes, Bull. Sci. Math., II. Ser. 100 (1976), p. 149-173.

[6] _ Nonlinear analysis on manifolds. Monge-Ampère equations, Springer, New York, Heidelberg, Berlin, 1982.

[7] R. Bartnik - The mass of an asymptotically flat manifold, Comm. Pure Appl. Math. 39 (1986), p. 661-693.

[8] R. Beig \& P. Chruściel - Killing Initial Data, Class. Quantum. Grav. 14 (1996), p. A83-A92, A special issue in honour of Andrzej Trautman on the occasion of his 64th Birthday, J.Tafel, editor.

[9] _ Killing vectors in asymptotically flat space-times: I. Asymptotically translational Killing vectors and the rigid positive energy theorem, Jour. Math. Phys. 37 (1996), p. 1939-1961, gr-qc/9510015.

[10] _ The isometry groups of asymptotically flat, asymptotically empty spacetimes with timelike ADM four-momentum, Commun. Math. Phys. 188 (1997), p. $585-597$, gr-qc/9610034. 
[11] R. Beig \& N. Ó. Murchadha - The Poincaré group as the symmetry group of canonical general relativity, Ann. Phys. 174 (1987), p. 463-498.

[12] R. Beig \& W. Simon - On the multipole expansion for stationary space-times, Proc. Roy. Soc. London A 376 (1981), p. 333-341.

[13] H. Bondi, M. van der Burg \& A. Metzner - Gravitational waves in general relativity VII: Waves from axi-symmetric isolated systems, Proc. Roy. Soc. London A 269 (1962), p. 21-52.

[14] G. Bunting \& A. Masood-ul-Alam - Nonexistence of multiple black holes in asymptotically euclidean static vacuum space-time, Gen. Rel. Grav. 19 (1987), p. $147-154$.

[15] D. Christodoulou - The boost problem for weakly coupled quasilinear hyperbolic systems of the second order, Jour. Math. Pures et Appl. 60 (1981), p. $99-130$.

[16] D. Christodoulou \& Y. Choquet-Bruhat - Elliptic systems in $H_{s, \delta}$ spaces on manifolds which are Euclidean at infinity, Acta. Math. 146 (1981), p. 129-150.

[17] D. Christodoulou \& N. Ó. Murchadha - The boost problem in general relativity, Comm. Math. Phys. 80 (1980), p. 271-300.

[18] P. Chruściel - On the relation between the Einstein and the Komar expressions for the energy of the gravitational field, Ann. Inst. H. Poincaré 42 (1985), p. 267282 .

[19] B B Bundary conditions at spatial infinity from a Hamiltonian point of view, in Topological Properties and Global Structure of Space-Time (P. Bergmann \& V. de Sabbata, eds.), Plenum Press, New York, 1986, pp. 49-59, URL http: //www.phys. univ-tours.fr/ piotr/scans.

[20] , On angular momentum at spatial infinity, Class. Quantum Grav. 4 (1987), p. L205-L210, erratum p. 1049.

[21] _ Quelques inégalités dans les espaces de Sobolev à poids, Tours preprint, unpublished, http://www.phys.univ-tours.fr/〜piotr/papers/wpi, 1987.

[22] Phys. 120 (1988), p. 233-248.

[23] P. Chruściel \& E. Delay - Existence of non-trivial asymptotically simple vacuum space-times, Class. Quantum Grav. 19 (2002), p. L71-L79, gr-qc/0203053, erratum Class. Quantum Grav. 19 (2002), 3389.

[24] _ Manifold structures for sets of solutions of the general relativistic constraint equations, gr-qc/0309001, 2003. 
[25] P. Chruściel \& M. Herzlich - The mass of asymptotically hyperbolic Riemannian manifolds, Pacific Jour. Math. (2001), in press, dg-ga/0110035.

[26] P. Chruściel \& O. Lengard - Solutions of wave equations in the radiating regime, Bull. Soc. Math. de France (2003), in press, math.AP/0202015.

[27] P. Chruściel \& R. MAzzeo - On "many-black-hole" vacuum spacetimes, Class. Quantum Grav. 20 (2003), p. 729-754, gr-qc/0210103.

[28] P. Chruściel \& G. Nagy - The Hamiltonian mass of asymptotically antide Sitter space-times, Class. Quantum Grav. 18 (2001), p. L61-L68, hepth/0011270.

[29] P. Chruściel \& W. Simon - Towards the classification of static vacuum spacetimes with negative cosmological constant, Jour. Math. Phys. 42 (2001), p. 17791817, gr-qc/0004032.

[30] J. Corvino - Scalar curvature deformation and a gluing construction for the Einstein constraint equations, Commun. Math. Phys. 214 (2000), p. 137-189.

[31] - lecture in Oberwolfach, July 2000.

[32] J. Corvino \& R. Schoen - On the asymptotics for the vacuum Einstein constraint equations, gr-qc/0301071, 2003.

[33] _ Vacuum spacetimes which are identically Schwarzschild near spatial infinity, talk given at the Santa Barbara Conference on Strong Gravitational Fields, June 22-26, 1999, http://doug-pc.itp.ucsb.edu/online/gravity_ c99/schoen/.

[34] S. DAIN - Initial data for stationary space-times near space-like infinity, Class. Quantum Grav. 18 (2001), p. 4329-4338, gr-qc/0107018.

[35] T. Damour \& B. SchmidT - Reliability of perturbation theory in general relativity, Jour. Math. Phys. 31 (1990), p. 2441-2453.

[36] I. Ekeland \& R. Temam - Convex analysis and variational problems, Studies in Math. and its Appl., vol. 1, North Holland, Amsterdam, 1976.

[37] H. Friedrich - On static and radiative spacetimes, Commun. Math. Phys. 119 (1988), p. $51-73$.

[38] _ Einstein equations and conformal structure: Existence of anti-de-Sittertype space-times, Jour. Geom. and Phys. 17 (1995), p. 125-184.

[39] _ Einstein's equation and geometric asymptotics, in Gravitation and Relativity: At the turn of the Millennium, Proceedings of GR15 (N. Dahdich \& J. Narlikar, eds.), IUCAA, Pune, 1998, p. 153-176.

[40] Conformal Einstein evolution, in Proceedings of the Tübingen Workshop on the Conformal Structure of Space-times (H. Friedrich \& J. Frauendiener, eds.), Lecture Notes in Physics, vol. 604, Springer, 2002, gr-qc/0209018, p. 1-50. 
[41] C. Graham \& J. Lee - Einstein metrics with prescribed conformal infinity on the ball, Adv. Math. 87 (1991), p. 186-225.

[42] V. Guillemin \& A. Pollack - Differential topology, Prentice-Hall, Englewood Cliffs, N.J, 1974.

[43] R. Hansen - Multipole moments of stationary space-times, Jour. Math. Phys. 15 (1974), p. 46-52.

[44] E. HeBey - Sobolev spaces on Riemannian manifolds, Lect. Notes in Math., vol. 1635, Springer, Berlin, 1996.

[45] J. Isenberg, R. Mazzeo \& D. Pollack - Gluing and wormholes for the Einstein constraint equations, Commun. Math. Phys. 231 (2002), p. 529-568, gr-qc/0109045.

[46] - On the topology of vacuum spacetimes, Annales Henri Poincaré (2003), in press, gr-qc/0206034.

[47] D. Joyce - Constant scalar curvature metrics on connected sums, Ph.D. Thesis, Lincoln College, Oxford, 2001, math.DG/0108022.

[48] J. KÁNNÁR - Hyperboloidal initial data for the vacuum Einstein equations with cosmological constant, Class. Quantum Grav. 13 (1996), p. 3075-3084.

[49] J. KLENK - Existence of stationary vacuum solutions of Einstein's equations in an exterior domain, Jour. Aust. Math. Soc., Ser. B 41 (1999), p. 231-247 (English).

[50] D. Kramer, H. Stephani, M. MacCallum \& E. Herlt - Exact solutions of Einstein's field equations, (E. Schmutzer, ed.), Cambridge University Press, Cambridge, 1980.

[51] O. Ladyzhenskaya, V. Solonnikov \& N. Ural'tseva - Linear and quasilinear equations of parabolic type. Translated from the Russian by S. Smith, Translations of Mathematical Monographs. 23. Providence, RI: American Mathematical Society. XI, 648 p. , 1968.

[52] J. LEE - Fredholm operators and Einstein metrics on conformally compact manifolds, math.DG/0105046, 2001.

[53] A. Lightman, W. Press, R. Price \& S. Teukolsky - Problem book in relativity and gravitation, Princeton University Press, Princeton, N.J., 1975.

[54] C. Morrey - Multiple integrals in the calculus of variation, Springer Verlag, Berlin, Heidelberg, New York, 1966.

[55] R. Myers \& M. Perry - Black holes in higher dimensional space-times, Ann. Phys. 172 (1986), p. 304-347. 
[56] T. Regge \& C. Teitelboim - Role of surface integrals in the Hamiltonian formulation of general relativity, Ann. Phys. 88 (1974), p. 286-318.

[57] O. Reula - On existence and behaviour of asymptotically flat solutions to the stationary Einstein equations, Commun. Math. Phys. 122 (1989), p. 615-624.

[58] R. SACHS - Gravitational waves in general relativity VIII. Waves in asymptotically flat space-time, Proc. Roy. Soc. London A 270 (1962), p. 103-126.

[59] U. SchaudT - On the Dirichlet problem for stationary and axisymmetric Einstein equations, Commun. Math. Phys. 190 (1998), p. 509-540.

[60] W. Simon \& R. Beig - The multipole structure of stationary space-times, Jour. Math. Phys. 24 (1983), p. 1163-1171.

[61] B. Smith \& G. Weinstein - On the connectedness of the space of initial data for the Einstein equations, Electron. Res. Announc. Am. Math. Soc. 6 (2000), p. $52-63$.

[62] V. Solovyev - Generator algebra of the asymptotic Poincare group in the general theory of relativity, Teor. i Mat. Fiz. 65 (1985), p. 400-414, in Russian; english translation avail. in Theor. Math. Phys. 1986, p. 1240.

[63] H. TRIEBEL - Interpolation theory, functions spaces and differential operators, North Holland, Amsterdam, 1978. 